Prepared in cooperation with the Minnesota Department of Transportation

\title{
Methods and Results of Peak-Flow Frequency Analyses for Streamgages in and bordering Minnesota, through Water Year 2011
}

Scientific Investigations Report 2013-5110 
Cover. U.S. Geological Survey streamgage 05132000 Big Fork River at Big Falls, Minnesota, April 10, 2011. Photograph by Russ Buesing, U.S. Geological Survey. 


\section{Methods and Results of Peak-Flow Frequency Analyses for Streamgages in and bordering Minnesota, through Water Year 2011}

By Erich W. Kessler, David L. Lorenz, and Christopher A. Sanocki

Prepared in cooperation with the Minnesota Department of Transportation

Scientific Investigations Report 2013-5110 


\title{
U.S. Department of the Interior SALLY JEWELL, Secretary
}

\section{U.S. Geological Survey Suzette M. Kimball, Acting Director}

\author{
U.S. Geological Survey, Reston, Virginia: 2013
}

For more information on the USGS - the Federal source for science about the Earth, its natural and living resources, natural hazards, and the environment, visit http://www.usgs.gov or call 1-888-ASK-USGS.

For an overview of USGS information products, including maps, imagery, and publications, visit http://www.usgs.gov/pubprod

To order this and other USGS information products, visit http://store.usgs.gov

Any use of trade, firm, or product names is for descriptive purposes only and does not imply endorsement by the U.S. Government.

Although this information product, for the most part, is in the public domain, it also may contain copyrighted materials as noted in the text. Permission to reproduce copyrighted items must be secured from the copyright owner.

Suggested citation:

Kessler, E.W., Lorenz, D.L., and Sanocki, C.A., 2013, Methods and results of peak-flow frequency analyses for streamgages in and bordering Minnesota, through water year 2011: U.S. Geological Survey Scientific Investigations Report 2013-5110, 43 p., http://pubs.usgs.gov/sir/2013/5110/. 


\section{Contents}

Abstract
Introduction.
Methods Used for Peak-Flow Frequency Analyses
$\quad$ Peak-Flow Data
Peak-Flow Statistics
Results of Peak-Flow Frequency Analyses
Explanation and Example of a Streamgage Report File
Summary
Appendion and Examples of Streamgage Graph Files
$\quad$ Appendix 1. Streamgages in and bordering Minnesota
$\quad$ Appendix 2. Streamgage Report Files
$\quad$ Appendix 3. Streamgage Graph Files

\section{Figures}

1. Map showing streamgages with peak-flow data in Minnesota, 2011 ..............................3

2. Example of a streamgage graph file with historical peak flows.......................................11

3. Example of a streamgage graph file with low-outlier threshold ......................................12

4. Example of a streamgage graph file with censored peak flows .....................................13

\section{Tables}

1. Example of page 1 of a streamgage report file .............................................................

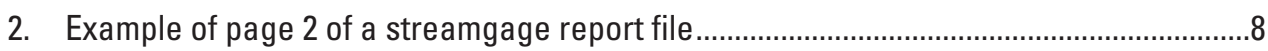

3. Example of page 3 and subsequent pages of a streamgage report file ...........................

4. Peak-flow codes used in analysis ...............................................................................

1-1. Streamgages in and bordering Minnesota with peak-flow data through

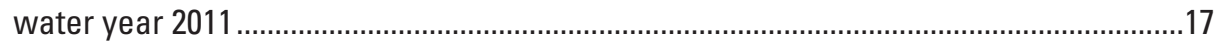




\title{
Conversion Factors
}

\begin{tabular}{ccl}
\multicolumn{1}{c}{ Inch/Pound to SI } & & \\
\hline \multicolumn{1}{c}{ Multiply } & By & To obtain \\
\hline square mile $\left(\mathrm{mi}^{2}\right)$ & Area & hectare $($ ha $)$ \\
square mile $\left(\mathrm{mi}^{2}\right)$ & 259.0 & square kilometer $\left(\mathrm{km}^{2}\right)$ \\
\hline & 2.590 & \\
\hline cubic foot per second $\left(\mathrm{ft}^{3} / \mathrm{s}\right)$ & Flow rate & cubic meter per second $\left(\mathrm{m}^{3} / \mathrm{s}\right)$ \\
\hline
\end{tabular}

Water year is the 12-month period October 1 through September 30, designated by the calendar year in which the water year ends.

Vertical coordinate information is referenced to the North American Vertical Datum of 1988 (NAVD 88).

Horizontal coordinate information is referenced to the North American Datum of 1983 (NAD 83). Altitude, as used in this report, refers to distance above the vertical datum.

\section{Abbreviations}

\author{
AEP annual exceedance probability \\ EMA expected moments algorithm \\ NWIS National Water Information System (U.S. Geological Survey database) \\ USGS U.S. Geological Survey \\ WIE weighting of independent estimates
}




\title{
Methods and Results of Peak-Flow Frequency Analyses for Streamgages in and bordering Minnesota, through Water Year 2011
}

\author{
By Erich W. Kessler, David L. Lorenz, and Christopher A. Sanocki
}

\section{Abstract}

Peak-flow frequency analyses were completed for 409 streamgages in and bordering Minnesota having at least 10 systematic peak flows through water year 2011. Selected annual exceedance probabilities were determined by fitting a log-Pearson type III probability distribution to the recorded annual peak flows. A detailed explanation of the methods that were used to determine the annual exceedance probabilities, the historical period, acceptable low outliers, and analysis method for each streamgage are presented. The final results of the analyses are presented.

\section{Introduction}

Knowledge of the peak-flow values associated with floods is essential for the design of bridges, culverts, and dams along Minnesota's rivers and streams. Statistical probability (chance) commonly is used to put a context to floods and their occurrence (Holmes and Dinicola, 2010). If the probability of a particular flood magnitude being equaled or exceeded is known, then risk can be assessed. Thus, peak-flow frequency analyses are needed for flood-plain management and flood insurance rate studies. These data also are used for regulation and planning of water resources.

The annual exceedance probability (AEP) is the probability that the largest peak flow during a water year (October 1 through September 30) equals or exceeds the associated flow. These probability estimates, in the past, have been reported in terms of a recurrence interval (the reciprocal of the AEP). For example, an AEP of 0.01 is associated with the flow that would be exceeded once in a random sample of 100 water years on average (100-year flood). The terminology associated with flood-frequency estimates has shifted away from recurrence interval, and the AEP is now the preferred terminology by the U.S. Geological Survey (USGS; Holmes and Dinicola, 2010). Throughout this report, the term AEP will be used to describe peak-flow frequency estimates.
For the purpose of estimating peak-flow frequencies, two types of streamgage record are used: continuous and partial. Continuous-record streamflow data are collected with sufficient frequency to define daily mean values and variations within a day. Partial-record data are discrete measurements of one or more hydrologic parameters that are obtained during a period of time. Common examples of partial-record data are peak stage (height of water surface above an established datum) and flow collected at a crest-stage gage, which is a streamgage that is nonmechanical, nontelemetered, and intended to record only the peak (crest) stream level since the last site visit. The longest record for a streamgage in Minnesota is for the Mississippi River at St. Paul, Minnesota. (USGS streamgage 05331000), which has continuous-record streamflow from 1893 through 2011 (U.S. Geological Survey, 2013a). Gaging of additional streams in Minnesota by the USGS to obtain daily streamflow records started about 1909. The number of streamgages decreased between 1912 and 1920. During the late 1920s and early 1930s, the number of streamgages increased and many of those streamgages have been in operation through 2011. During the years, streamgages were added to the USGS streamgage network where streamflow information was needed or were discontinued where additional data were less critical. In Minnesota, most continuous-recording streamgages are located on perennial (continuously flowing) streams with drainage areas greater than 300 square miles $\left(\mathrm{mi}^{2}\right)$. For example, in 2008 about 82 percent of continuous-recording streamgages in Minnesota had drainage areas greater than $300 \mathrm{mi}^{2}$.

In the 1950s, planners for the Interstate Highway System learned that little peak-flow information was available for streams with drainage areas less than about $60 \mathrm{mi}^{2}$. This information was needed to determine the sizes of bridge and culvert openings to use where highways crossed stream channels. As a result, small-stream flood investigations were initiated nationwide. The program in Minnesota began in 1958, and during the next 6 years about 150 crest-stage gages were established to determine annual peak flow and stage on streams draining about $60 \mathrm{mi}^{2}$ or less (Gunard and Smith, 1982). These creststage gages differ from continuous-record streamgages in that 
streamflow record generally is not continuous-only peak stages and flows are recorded. Most of these crest-stage gages were operated through the 1970s. In the 1980s, crest-stage gages with drainage areas less than $10 \mathrm{mi}^{2}$ were discontinued. Recently (1997), new crest-stage gages were established throughout the State on streams draining areas from $10 \mathrm{mi}^{2}$ to several hundred square miles.

Lorenz and others (2010) presented peak-flow frequency analyses for 330 streamgages in Minnesota and adjacent areas in Iowa and South Dakota based on data through water year 2005. The USGS, in cooperation with the Minnesota Department of Transportation, completed updated or new analyses of peak-flow frequency for 409 streamgages in and bordering Minnesota using data through water year 2011.

The purpose of this report is to describe the methods used in peak-flow frequency analyses to determine the peak-flow statistics (AEPs) for streamgages in and bordering Minnesota, through water year 2011. Specifically, the report includes a list of the streamgages used in this study, describes the methods used to graph the statistics, and describes the graphs and output files.

\section{Methods Used for Peak-Flow Frequency Analyses}

Peak-flow data from 530 streamgages in and bordering Minnesota (fig. 1, table 1-1 in appendix 1) were retrieved from the USGS National Water Information System (NWIS; Mathey, 1998). Peak-flow records for these sites are available from the NWIS internet interface, NWISWeb, at $h t t p: / / n w i s$. waterdata.usgs.gov/nwis/peak. The peak-flow data for the period of record for each streamgage through September 30, 2011, were used in this analysis. Peak-flow frequency analyses were determined for 409 of the 530 streamgages. The remaining 121 stations did not have the minimum 10 systematic recorded peak flows required for statistical analysis.

\section{Peak-Flow Data}

Annual peak flow is defined as the greatest instantaneous flow observed during the water year (October 1 through September 30). The annual peak flow must be independent of annual peak flows that occurred during adjacent water years.

Peak-flow data can be divided into two general categories: systematic and historical. Systematic peak flows are data that are collected whenever the streamgage is operational, even if only in operation for 1 year. These data are assumed to represent a systematic random sample of the population of peak flows. Historical peak flows are data that are collected for the specific purpose of documenting a large flood at a location where or at a time when no streamgage is operating. Historical peak flows represent a nonrandom sample and must be treated separately from systematic peak flows.
Because systematic peak flows are assumed to be a random sample of the population, their analysis is straightforward - the statistics of the population are based on the sample of systematic peak flows. Historical peak flows can yield additional information if they can be put into the context of a historical period. If a historical peak flow was recorded because it was an extraordinary event, then it can be put into the context of the historical period based on recorded human events (newspapers for example). In some cases, historical peak flows at a short-term streamgage can be correlated with record from a long-term streamgage to estimate a historical period. Examples are described in the "Results of Peak-Flow Frequency Analyses" section.

\section{Peak-Flow Statistics}

Selected AEP statistics were determined by fitting a log-Pearson Type III probability distribution to the recorded annual peak flows according to the procedures outlined in Bulletin 17B (Interagency Advisory Committee on Water Data, 1982). The latest version of the computer program PEAKFQ (Flynn and others, 2006) was used to compute the frequency estimates for the streamgages. The procedures in Bulletin 17B include the Bulletin 17B Grubbs-Beck test (Grubbs and Beck, 1972; based on the mean, standard deviation, and skew) for detecting unusually high and low peak flows (outliers) at the 10-percent significance level that can affect the analysis. Peak flows flagged as high outliers require user judgment about putting the outlier into a historical period of record. Peak flows flagged as low outliers generally are excluded from the analysis, and the conditional probability adjustment described in appendix 5 of Bulletin 17B (Interagency Advisory Committee on Water Data, 1982) is applied to the analysis. Bulletin $17 \mathrm{~B}$ recommends the use of a skew coefficient that is based on the skew of the log-series of the period of record (commonly termed the "station skew"), weighted with a generalized, or regional, skew coefficient. The weighting is based on the length of the period of record and the estimated standard error for the method used to determine the generalized skew coefficient. The generalized skew coefficients for Minnesota streamgages used in the peak-flow frequency analysis for this report were based on Lorenz (1997).

An alternate method, the expected moments algorithm (EMA; Cohn and others, 1997), was used to reduce bias that results from the method described in Bulletin 17B. Both methods assume the peak flows have a log-Pearson Type III distribution, which is characterized by the mean, standard deviation, and skewness of the log-transformed peak-flow values; however, the EMA permits efficient use of interval data, which arise in the context of historical flood information (Cohn and others, 1997), low outliers (Griffis and others, 2004), uncertain data points (Cohn and others, 2001), and other selected situations. The EMA also provides a method for computing reasonably accurate confidence intervals (Cohn and others, 2001). Confidence intervals provide indications of 


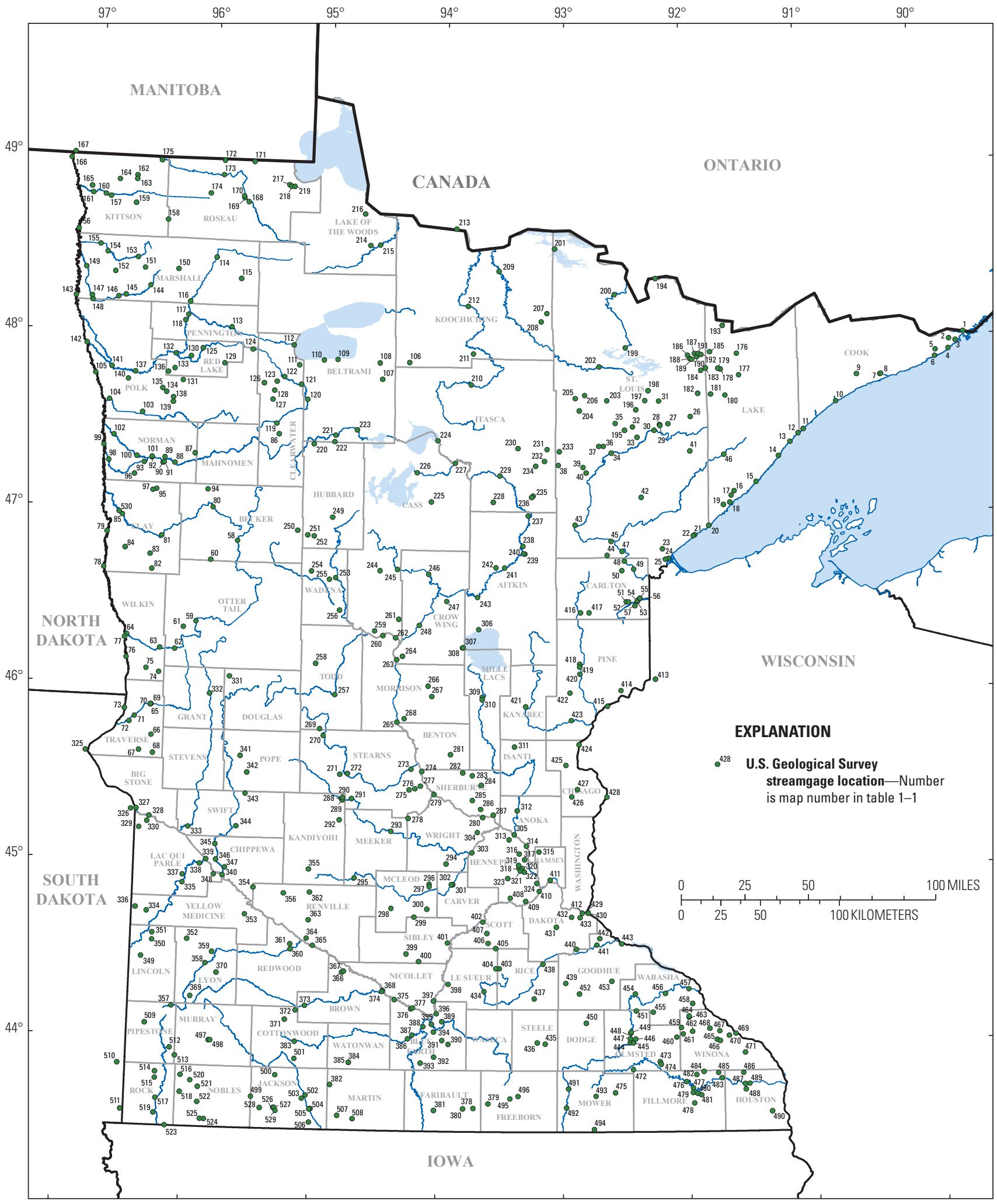

Base from U.S. Census Bureau's Master Address File Topologically Integrated Geographic Encoding and Referencing (MAF/TIGER) database, 2009,

Minnesota Department of Natural Resources digital data, 2012,

Esri Data and Maps, 2008, various scales

Universal Transverse Mercator projection, Zone 15

North American Datum of 1983

Figure 1. Streamgages with peak-flow data in Minnesota, 2011. 
the ability to predict a certain AEP and generally, the longer the period of record or number of peak flows documented, the more reliable the AEP. The program that was used, PeakFqSA (http://www.timcohn.com/TAC_Software/PeakfqSA/, accessed June 2010), retains the structure and moments-based approach of Bulletin 17B and therefore, easily accommodates the other Bulletin 17B procedures (Cohn and others, 1997; Cohn and others, 2001; England, Jarrett, and Salas, 2003; England, Salas, and Jarrett, 2003; Griffis and others, 2003, 2004).

PeakFqSA uses a single Grubbs-Beck test that is the same as the Bulletin 17B Grubbs-Beck test except that PeakFqSA computes an attained probability value for the lowest flow instead of the 10-percent significance level used in Bulletin 17B. PeakFqSA also includes an additional test, the multiple Grubbs-Beck test, for low outliers (Timothy A. Cohn, U.S. Geological Survey, written commun., June 2011), which is intended to reduce the effect of low peak flows on the upper part of the frequency curve. This test is based on a one-sided, 10-percent significance level for a normally distributed sample, but the test is constructed so that groups of ordered data are examined (for example, the eight smallest values) and excluded from the dataset when the critical value is calculated. If the critical value is greater than the eighth smallest value, then all eight values are considered to be low outliers. As described by Timothy A. Cohn (U.S. Geological Survey, written commun., February 2011) in an evaluation study, the low outliers identified by the multiple Grubbs-Beck test closely match user-selected low-outlier thresholds determined from plotted flood-frequency curves. The multiple Grubbs-Beck test was used in this analysis when a visual inspection of plotted flood-frequency curves identified that the upper tail of the fitted curve was unduly affected by several small peak flows that were not detected by the single Grubbs-Beck test, but should probably not be used in the analysis. For cases where the multiple Grubbs-Beck test detected too many low outliers, a fixed low-outlier threshold was supplied by the user to improve the frequency curve fit.

The decisions regarding the use of generalized skew, handling of low and high outliers, and handling of peak flows affected by regulation or urbanization are outlined below, and details are provided for each station in table 1-1 in appendix 1 .

- The selection of skew was based on two factors: drainage area and degree of regulation. The station skew was used for streamgages with drainage areas larger than $6,000 \mathrm{mi}^{2}$ or with peak flows affected by regulation or urbanization. For streamgages with drainage areas less than $6,000 \mathrm{mi}^{2}$ and peak flows substantially unaffected by regulation or urbanization, the weighted skew, including the station skew and the generalized skew (Lorenz, 1997), was used.

- The default procedures of Bulletin 17B were used for analyses of each streamgage unless the following conditions were encountered:

$\circ$ If the default procedures in Bulletin 17B detected a low outlier, then the EMA procedure was used to analyze the peak flows. If the single Grubbs-Beck test result fit the data well, then it was accepted (this result is considered the default EMA result in table 1-1 in appendix 1). Otherwise, the multiple GrubbsBeck test or a fixed low-outlier threshold was used to fit the data (these two results are considered the modified EMA result in table 1-1 in appendix 1).

$\circ$ If the default procedures in Bulletin 17B detected a high outlier, then records for nearby, long-term streamgages were reviewed to identify an appropriate historical context for the suspected outlier. If a similar peak flow was present, then the high outlier was placed in that historical period. Otherwise, the results were accepted as is, and it was noted that insufficient evidence existed to place the peak flow in a historical context.

- Peak flows affected by regulation or urbanization present a special problem for peak-flow analysis. The general assumption required is that the management of the regulation has been consistent over time so that the results of the analysis do reflect the current flood statistics. That assumption was made for all streamgages except when regulation started or stopped during the systematic period, in which case the recent record was used for the analysis.

The AEPs also were calculated using the weighting of independent estimates (WIE) procedure (Interagency Advisory Committee on Water Data, 1982, appendix 8). The independent estimates were based on Lorenz and others (2010). The WIE procedure was applied to appropriate records as described in Lorenz and others (2010): unregulated, unaffected by urbanization, and drainage area less than $3,000 \mathrm{mi}^{2}$. The WIE procedure involves using the at-site estimates as well as those from regional studies. The use of WIE can provide a better estimate of the AEP at a streamgage by computing a weighted average of the at-site estimate with the regional estimate. 


\section{Results of Peak-Flow Frequency Analyses}

Results of the peak-flow analyses for each of the 409 streamgages for which peak-flow frequency analyses were completed are presented in appendixes 2 and 3. This section describes the information presented in these appendixes.

For each streamgage, a streamgage report file (appendix 2) and a streamgage graph file (appendix 3 ) are provided in portable document format (PDF). An example of the streamgage report file is shown in tables $1-3$. The streamgage report files present the specifications of the analyses, the results of the peak-flow analyses, and the peak-flow data. These report files summarize information from all analytical methods. An example of several different streamgage graph files are depicted in figures $2-4$. These figures present the data and the results of the analysis on two probability scales: the normal scale is useful for assessing the curvature of the data, and the Pearson Type III is useful for assessing the goodness of fit.

\section{Explanation and Example of a Streamgage Report File}

Each streamgage report file has three or more pages. The first page summarizes the data and presents the options for analysis and the results of the systematic record analysis, which basically includes the raw statistics of the peakflow data. The second page presents the final analysis, and the remaining page or pages present the peak-flow data. An example of the contents of the first page of the streamgage report file is shown in table 1 with comments and explanations of the report file's contents.

An example of the contents of the second page of the streamgage report file is shown in table 2 . This page of the report file explains the results of the final analysis, which contains the moments of the common logarithms of the peak flows and the estimated peak flows at selected probability levels.
An example of the third page and subsequent pages, if any, of the streamgage report file is shown in table 3. This page or pages of the report file provide the peak-flow data for each year of streamgage record and any qualifying codes used in the analysis. Possible peak-flow codes used in analysis are shown in table 4.

\section{Explanation and Examples of Streamgage Graph Files}

Each streamgage graph file is composed of one page containing two graphs: one normal and one Pearson type III (appendix 3). Each graph has the peak-flow frequency estimate (with respective confidence limits) and the annual peak flows. If applicable, the graphs also indicate historical peak flows (blue dots), historical thresholds, low-outlier thresholds, and peak flows with " $<$ " and " >" symbols (shown as censored peak flows) with their respective direction. Example graphs are shown for historical peak flows (fig. 2), low-outlier threshold (fig. 3), and censored peak flows (fig. 4).

\section{Summary}

Knowledge of the peak-flow values associated with floods is essential for the design of bridges, culverts, and dams along Minnesota's rivers and streams. Peak-flow frequency analyses were completed by the U.S. Geological Survey, in cooperation with the Minnesota Department of Transportation, for 409 streamgages in and bordering Minnesota having at least 10 systematic peak flows through water year 2011. The results of the peak-flow frequency analyses are presented in this report. Selected annual exceedance probabilities were determined by fitting a log-Pearson Type III probability distribution to the recorded annual peak flows according to the procedures outlined in Bulletin 17B. A detailed explanation of the methods that were used to determine the annual exceedance probabilities, the historical period, acceptable low outliers, and analysis method at each streamgage are presented. The final results of the analyses are presented in tables and graphs. 
Table 1. Example of page 1 of a streamgage report file.

\section{ANNUAL PEAK-FLOW FREQUENCY ANALYSIS}

For more information on the contents of this documentation, see Kessler and others (2013). ${ }^{1}$

Streamgage number and name:

05247500 Crow Wing River near Pillager, Minn.

Peak-flow information:

Number of systematic peak flows in record

Systematic period begins ${ }^{2}$

1969

Systematic period ends

Length of systematic record

Years without information

Number of historical peak flows in record

Length of historical period

Historical period begins ${ }^{6}$

Historical period ends

Historical period based on

Frequency analysis options:

$\begin{array}{ll}\text { Method } & \text { Expected moments algorithm (EMA) } \\ \text { Skew option } & \text { Station skew }{ }^{9} \\ \text { Low-outlier method } & \text { Multiple Grubbs-Beck test }{ }^{10}\end{array}$

Bulletin 17B systematic record analysis results:

Moments of the common logarithms of the peaks: ${ }^{11}$

\begin{tabular}{ccr} 
Mean & $\begin{array}{c}\text { Standard } \\
\text { deviation }\end{array}$ & \multicolumn{1}{c}{ Skewness } \\
3.7765 & 0.2636 & -0.250
\end{tabular}

Outlier criteria and number of peak flows exceeding: ${ }^{12}$

$\begin{array}{rrr}\text { Low } & 1,173.7 & 0 \\ \text { High } & 18,300.0 & 0\end{array}$

EMA systematic record analysis results:

Moments of the common logarithms of the peaks: ${ }^{13}$

$\begin{array}{lcr}\text { Mean } & \begin{array}{c}\text { Standard } \\ \text { deviation }\end{array} & \text { Skewness } \\ 3.7759 & 0.2592 & -0.312\end{array}$

Low-outlier information:

Number of low outliers

0

Low-outlier threshold $\quad$ Not determined $^{14}$

${ }^{1}$ This is a link to this report.

${ }^{2}$ The first and last year of the systematic record is given. There can be breaks in the record not shown by these data.

${ }^{3}$ This is the number of years between the first and last years of systematic record that do not have peak-flow data. 


\section{Table 1. Example of page 1 of a streamgage report file.-Continued}

${ }^{4}$ If this number is greater than 0 , then the years of the historical peaks are listed to the right.

${ }^{5}$ If a historical period analysis is included in the analysis, then this information and the next three lines are included in the report. Otherwise the next subsection follows immediately.

${ }^{6}$ The beginning and end of the historical period are expressed as dates for EMA, but they are not needed for Bulletin 17B and "Not used by Bulletin 17B" is printed.

${ }^{7} \mathrm{~A}$ brief explanation for the definition of the historical period is given here; more detail is available in table $1-1$ in appendix 1.

${ }^{8}$ This will be either "Expected moments algorithm (EMA)" or "Bulletin 17B," depending on the method used.

${ }^{9}$ The skew option is either "station skew" or "weighted." In most cases, "weighted" is used to compute the final skewness according to the guidelines in Bulletin 17B. "Station skew" is used in cases when the drainage area is too large for the generalized skewness (Lorenz, 1997) or when peak flows are affected by regulation. The EMA method also computes the final skew according to the guidelines in Bulletin 17B, but uses a different computation for the variance of the station skewness.

${ }^{10}$ The low-outlier method is one of the following "multiple Grubbs-Beck test," "single Grubbs-Beck test," "fixed," or "Bulletin 17B Grubbs-Beck test." The first two are options for the EMA method.

${ }^{11}$ The mean, standard deviation, and skewness are computed from the systematic record, not the historical record.

${ }^{12}$ The low- and high-outlier criteria are computed from the mean and standard deviation, with scaling controlled by the number of observations. If any peak flow was less than the low-outlier criterion, then EMA was used. If any peak flows exceeded the high-outlier criterion, then an attempt was made to find a historical context for those peak flows.

${ }^{13}$ The mean, standard deviation, and skewness are computed from the historical period for EMA but not for Bulletin 17B. EMA does not distinguish between the systematic record and the historical record as do the methods in Bulletin 17B.

${ }^{14}$ EMA does not report a low-outlier threshold if no peak flow is less than the criteria of the test. If any peak flows are less than the criteria of the test, then the threshold is computed as the value of the smallest peak flow not exceeding the criteria. 
Table 2. Example of page 2 of a streamgage report file.

Final analysis results:

Moments of the common logarithms of the peak flows: ${ }^{1}$

$\begin{array}{ccc}\text { Mean } & \begin{array}{c}\text { Standard } \\ \text { deviation }\end{array} & \text { Skewness } \\ 3.7759 & 0.2592 & -0.312\end{array}$

Annual frequency curve at selected exceedance probabilities: ${ }^{2}$

$\begin{array}{rrrr}\text { Exceedance } & \text { Peak } & \text { Lower 95 } & \text { Upper 95 } \\ \text { probability } & \text { estimate } & \text { level } & \text { level } \\ 0.9950 & 1,080 & 253 & 1,690 \\ 0.9900 & 1,300 & 375 & 1,930 \\ 0.9500 & 2,130 & 1,080 & 2,820 \\ 0.9000 & 2,730 & 1,760 & 3,480 \\ 0.8000 & 3,650 & 2,730 & 4,530 \\ 0.6667 & 4,740 & 3,760 & 5,830 \\ 0.5000 & 6,160 & 5,010 & 7,570 \\ 0.4292 & 6,840 & 5,600 & 8,400 \\ 0.2000 & 9,930 & 8,200 & 11,900 \\ 0.1000 & 12,500 & 10,300 & 15,100 \\ 0.0400 & 15,900 & 13,000 & 20,500 \\ 0.0200 & 18,400 & 14,800 & 25,500 \\ 0.0100 & 20,800 & 16,300 & 31,100 \\ 0.0050 & 23,300 & 17,400 & 37,300 \\ 0.0020 & 26,600 & 18,500 & 46,600\end{array}$

${ }^{1}$ The mean, standard deviation, and skewness are computed from the historical period, if any, and incorporate the generalized skew, if appropriate.

${ }^{2}$ If the weighting of independent estimate (WIE) procedure was used, then three additional columns are displayed in this table: WIE estimate, lower-95 WIE level, and upper-95 WIE level. The WIE estimates are made only for the $0.500,0.200,0.100,0.040,0.020,0.010$, and 0.002 exceedance probabilities.

${ }^{3}$ The lower-95 level is the lower 95-percent confidence limit for the estimated peak flow. The upper-95 level is the upper 95 -percent confidence limit for the estimated peak flow. 
Table 3. Example of page 3 and subsequent pages of a streamgage report file.

Peak-flow data available in the analysis:

Explanation of symbols and codes ${ }^{1}$

$\mathrm{H}$ Historical, outside of systematic record

$\mathrm{K}$ Peak flow affected by regulation

1924 to 1968 lower perception threshold²: 18,300

\begin{tabular}{|c|c|c|c|c|c|}
\hline Water & Peak & Peak & Water & Peak & Peak-flow \\
\hline year & flow & code & year & flow & code \\
\hline 1965 & 18,300 & $\mathrm{KH}$ & 1989 & 6,370 & K \\
\hline & $\left({ }^{3}\right)$ & & 1990 & 4,270 & $\mathrm{~K}$ \\
\hline 1969 & 16,600 & $\mathrm{~K}$ & 1991 & 3,380 & K \\
\hline 1970 & 6,380 & $\mathrm{~K}$ & 1992 & 2,880 & K \\
\hline 1971 & 7,500 & K & 1993 & 7,360 & K \\
\hline 1972 & 11,700 & K & 1994 & 6,260 & K \\
\hline 1973 & 6,140 & K & 1995 & 6,600 & K \\
\hline 1974 & 12,200 & K & 1996 & 13,900 & K \\
\hline 1975 & 10,800 & K & 1997 & 16,100 & K \\
\hline 1976 & 4,470 & $\mathrm{~K}$ & 1998 & 4,240 & K \\
\hline 1977 & 1,520 & K & 1999 & 10,800 & K \\
\hline 1978 & 4,220 & $\mathrm{~K}$ & 2000 & 3,940 & K \\
\hline 1979 & 7,970 & K & 2001 & 17,500 & K \\
\hline 1980 & 2,390 & K & 2002 & 5,310 & K \\
\hline 1981 & 1,420 & K & 2003 & 7,340 & K \\
\hline 1982 & 5,450 & K & 2004 & 3,150 & K \\
\hline 1983 & 4,310 & $\mathrm{~K}$ & 2005 & 6,460 & $\mathrm{~K}$ \\
\hline 1984 & 3,940 & K & 2006 & 7,320 & K \\
\hline 1985 & 5,650 & K & 2007 & 5,820 & $\mathrm{~K}$ \\
\hline 1986 & 10,800 & K & 2008 & 4,550 & K \\
\hline \multicolumn{3}{|c|}{ Gap in systematic record ${ }^{4}$} & 2009 & 13,400 & K \\
\hline \multirow[t]{2}{*}{1988} & 2980 & $\mathrm{~K}$ & 2010 & 11,100 & K \\
\hline & & & 2011 & 7,560 & \\
\hline
\end{tabular}

${ }^{1}$ See table 4 for listing of all the symbols and codes.

${ }^{2}$ Perception threshold is based on an investigation of a historical period to determine whether the known historical peak flow had been exceeded in any other year.

${ }^{3}$ Blank spacing indicates separation between historical and systematic periods.

${ }^{4}$ Indicates a gap in the systematic record, as a result of a discontinued streamgage or missing peak flow. 
Table 4. Peak-flow codes used in analysis.

[NWIS, U.S. Geological Survey National Water Information System]

\begin{tabular}{|c|c|c|c|}
\hline $\begin{array}{l}\text { Peak-flow } \\
\text { code }\end{array}$ & Definition & $\begin{array}{l}\text { NWIS } \\
\text { code }\end{array}$ & Peak-flow file explanation \\
\hline $\mathrm{H}$ & Historical, outside of systematic record & 7 & Discharge is a historical peak flow. \\
\hline $\mathrm{U}$ & Peak flow affected by urbanization & $\mathrm{C}$ & $\begin{array}{l}\text { All or part of the record affected by urbanization, mining, } \\
\text { agricultural changes, channelization, or other. }\end{array}$ \\
\hline$<$ & Peak flow less than minimum recordable discharge & 4 & $\begin{array}{l}\text { Discharge less than indicated value, which was minimum } \\
\text { recordable discharge at this site. }\end{array}$ \\
\hline$>$ & Peak flow greater than maximum recordable discharge & 8 & Discharge actually greater than indicated value. \\
\hline $\mathrm{D}$ & Dam failure, non-recurrent flow anomaly & 3 & Discharge affected by dam failure. \\
\hline
\end{tabular}


05054000 Red River of the North at Fargo, N. Dak.
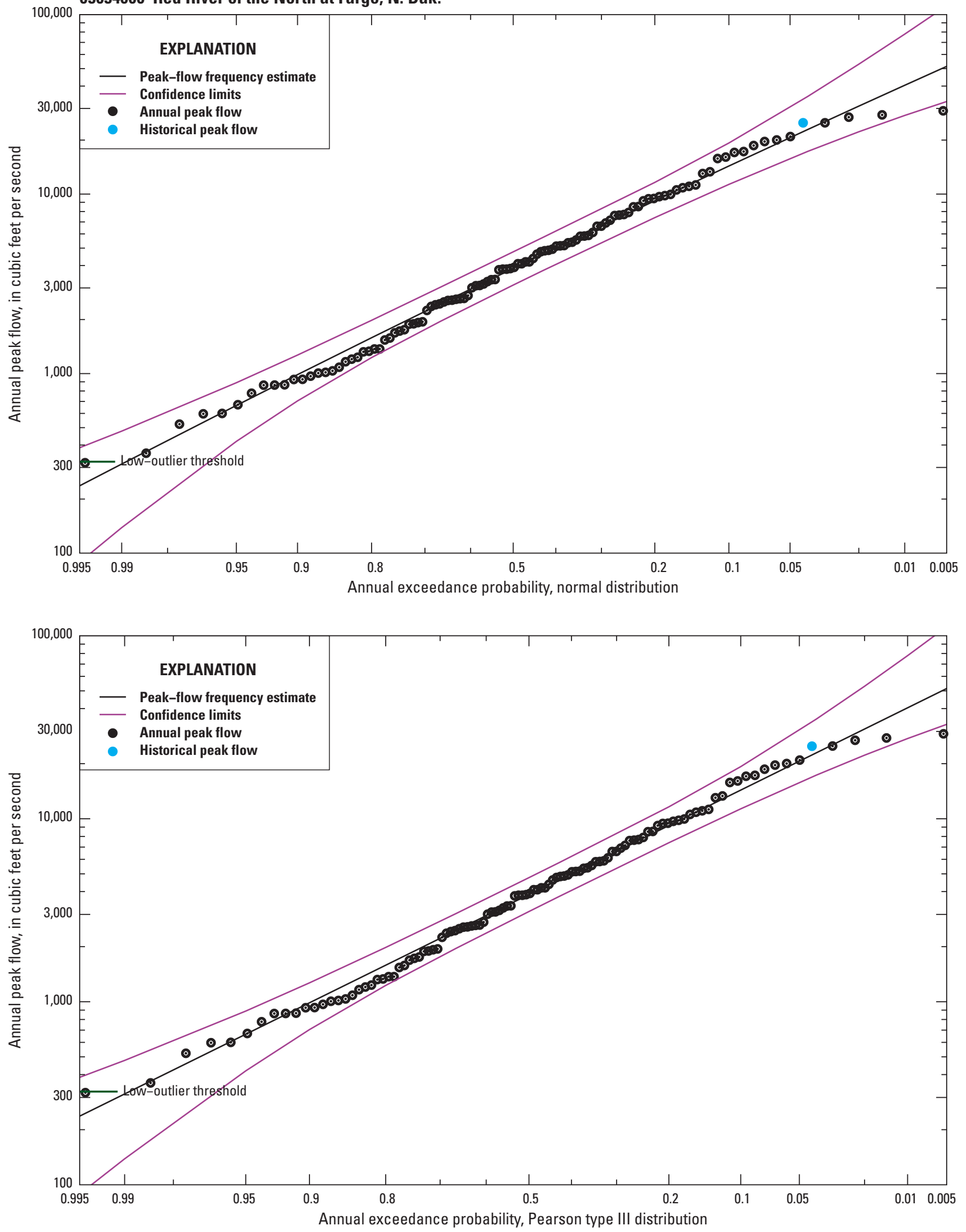

Figure 2. Example of a streamgage graph file with historical peak flows. 
05276100 North Fork Crow River tributary near Paynesville, Minn.
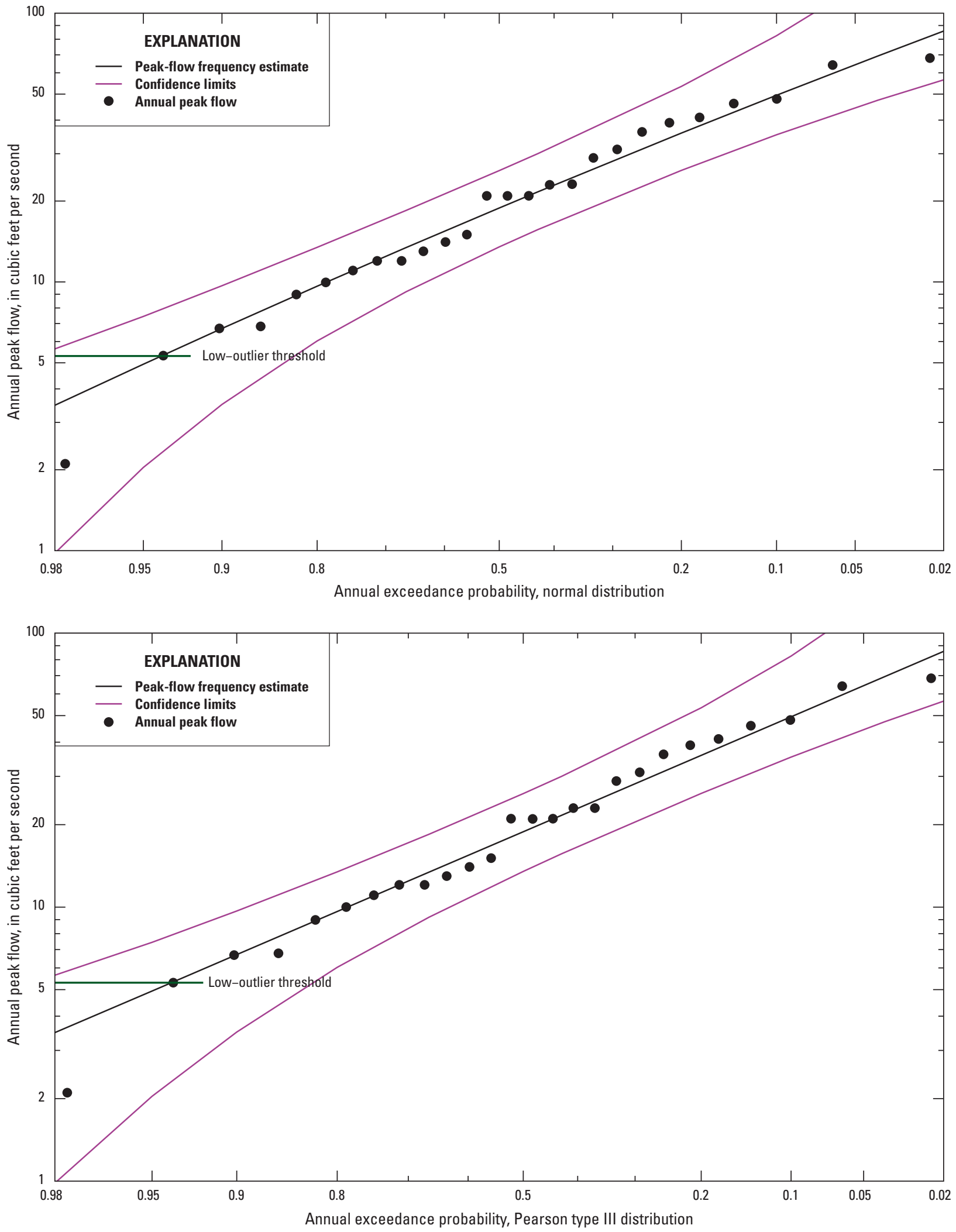

Figure 3. Example of a streamgage graph file with low-outlier threshold. 
05278120 North Fork Crow River near Kingston, Minn.
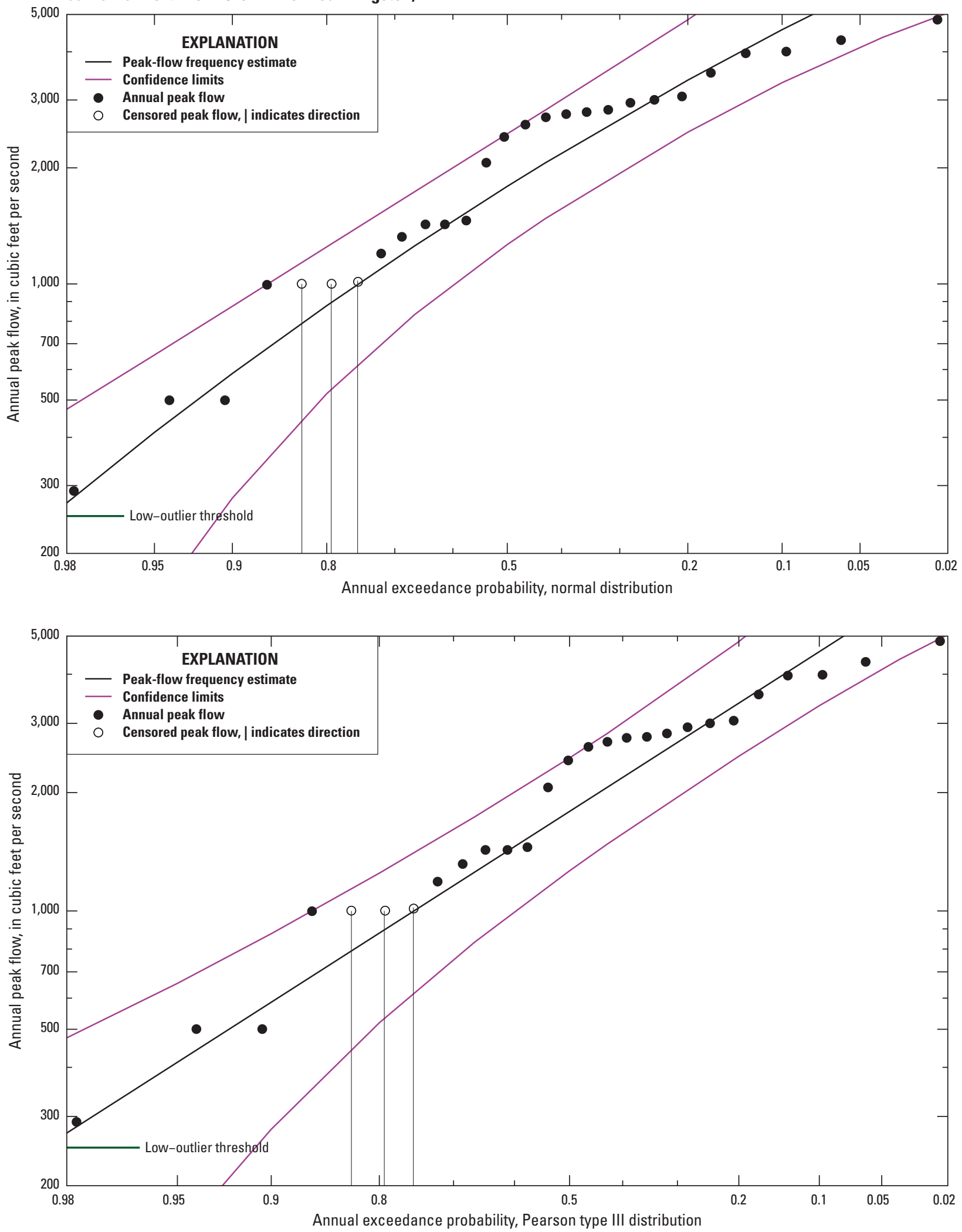

Figure 4. Example of a streamgage graph file with censored peak flows. 


\section{References Cited}

Cohn, T.A., Lane, W.L., and Baier, W.G., 1997, An algorithm for computing moments-based flood quantile estimates when historical flood information is available: Water Resources Research, v. 33, no. 9, p. 2089-2096. (Also available at $h t t p: / / d x$. doi.org/10.1029/97WR01640.)

Cohn, T.A., Lane, W.M., and Stedinger, J.R., 2001, Confidence intervals for expected moments algorithm flood quantile estimates: Water Resources Research, v. 37, no. 6, p. 1695-1706. (Also available at $h t t p: / / d x$.doi. org/10.1029/2001WR900016.)

England, J.F., Jarrett, R.D., and Salas, J.D., 2003, Databased comparisons of moments estimators using historical and paleoflood data: Journal of Hydrology, v. 278, no. 1, p. 172-196. (Also available at $h t t p: / / d x . d o i . o r g / 10.1016 /$ S0022-1694(03)00141-0.)

England, J.F., Salas, J.D., and Jarrett, R.D., 2003, Comparisons of two moments-based estimators that utilize historical and paleoflood data for the log Pearson type III distribution: Water Resources Research, v. 39, no. 9, p. 1243. (Also available at $h t t p: / / d x$.doi.org/10.1029/2002WR001791.)

Flynn, K.M., Kirby, W.H., Mason, R., and Cohn, T.A., 2006, Estimating magnitude and frequency of floods using the PeakFQ program: U.S. Geological Survey Fact Sheet 2006-3143, 1 sheet. (Also available at http://pubs.usgs.gov/ $f_{S} / 2006 / 3143 /$.)

Griffis, V.W., Stedinger, J.R., and Cohn, T.A., 2003, Extension of EMA to address regional skew and low outliers: Proceedings of the World Water \& Environmental Resources Congress 2003, June 23-26, 2003, Philadelphia, Pennsylvania. (Also available at $h t t p: / / d x . d o i$. org/10.1061/40685(2003)103.)

Griffis, V.W., Stedinger, J.R., and Cohn, T.A., 2004, Log Pearson type 3 quantile estimators with regional skew information and low outlier adjustments: Water Resources Research, v. 40, no. 7, W07503. (Also available at $h t t p: / /$ dx.doi.org/10.1029/2003WR002697.)

Grover, N.C., Lamb, W.A., Soulé, S.B., Spiegel, J.B., Grosbach, H.E., and Beckman, H.C., 1929, Surface water supply of the United States, 1925 - Part V. Hudson Bay and upper Mississippi River Basins: U.S. Geological Survey WaterSupply Paper 605, 179 p.
Grubbs, F.E., and Beck, Glenn, 1972, Extension of sample sizes and percentage points for significance tests of outlying observations: Technometrics, v. 14, no. 4, p. 847-854. (Also available at $h t t p: / / w w w . j s t o r . o r g / s t a b l e / 1267134$.

Gunard, K.T., and Smith, C.J., 1982, Small-stream flood investigations in Minnesota, October 1958 to September 1980: U.S. Geological Survey Open-File Report 82-433, 221 p. (Also available at http://pubs.er.usgs.gov/publication/ ofr 82433 .)

Holmes, R.R., Jr., and Dinicola, K., 2010, 100-Year flood-It's all about chance: U.S. Geological Survey General Information Product 106, 1 p. (Also available at http://pubs.usgs. gov/gip/106/.)

Interagency Advisory Committee on Water Data, 1982, Guidelines for determining flood-flow frequency: Bulletin 17B of the Hydrology Subcommittee, Office of Water Data Coordination, U.S. Geological Survey, 183 p.

Lorenz, D.L., 1997, Generalized skew coefficients for floodfrequency analysis in Minnesota: U.S. Geological Survey Open-File Report 97-4089, 15 p. (Also available at http:// pubs.usgs.gov/wri/1997/4089/report.pdf.)

Lorenz, D.L., Sanocki, C.A., and Kocian, M.J., 2010, Techniques for estimating the magnitude and frequency of peak flows on small streams in Minnesota based on data through water year 2005: U.S. Geological Survey Scientific Investigations Report 2009-5250, 54 p., accessed March 6, 2013, at $h t t p: / / p u b s . u s g s . g o v / s i r / 2009 / 5250 /$.

Mathey, S.B., ed., 1998, National water information system (NWIS): U.S. Geological Survey Fact Sheet FS-027-98, 2 p. (Also available at $h t t p: / / p u b s . u s g s . g o v / f s / F S-027-98 /$.)

U.S. Geological Survey, 2013a, National Water Information System (NWISWeb): U.S. Geological Survey database, accessed January 21, 2013, at http://waterdata.usgs.gov/ nwis/peak.

U.S. Geological Survey, 2013b, StreamStats-Minnesota: accessed February 21, 2013, at http://streamstats.usgs.gov/ minnesota.html. 
Appendixes 


\section{Appendix 1. Streamgages in and bordering Minnesota}

A total of 530 streamgages in and bordering Minnesota have peak-flow data through water year 2011 (table 1-1). Of these 530 streamgages, 409 had sufficient peak-flow data to compute peak-flow frequency analyses. The remaining 121 streamgages did not have the minimum 10 systematic recorded peak flows required for statistical analysis. 
Table 1-1. Streamgages in and bordering Minnesota with peak-flow data through water year 2011.

[Bulletin 17B method (Interagency Advisory Committee on Water Data, 1982); EMA, expected moments algorithm (Cohn and others, 1997); **/**, month and day of occurrence unknown]

\begin{tabular}{|c|c|c|c|c|c|c|}
\hline $\begin{array}{c}\text { Map } \\
\text { number } \\
\text { (fig. 1) }\end{array}$ & $\begin{array}{l}\text { Streamgage } \\
\text { number }\end{array}$ & Streamgage name & $\begin{array}{l}\text { Start of } \\
\text { systematic } \\
\text { record }\end{array}$ & $\begin{array}{l}\text { End of } \\
\text { systematic } \\
\text { record }\end{array}$ & Peak-flow analysis comments & $\begin{array}{l}\text { Weighting of independent } \\
\text { estimates (WIE) calculated? }\end{array}$ \\
\hline 1 & 04010500 & $\begin{array}{l}\text { Pigeon River at Middle Falls near Grand Portage, } \\
\text { Minn. }\end{array}$ & $05 / 19 / 1924$ & $05 / 01 / 2011$ & $\begin{array}{l}\text { Low outlier(s) flagged by Bulletin 17B method. Accepted modi- } \\
\text { fied results from EMA }\end{array}$ & No, large drainage basin. \\
\hline 2 & 04010505 & Maud Creek near Grand Portage, Minn. & $10 / 23 / 2004$ & $10 / 23 / 2004$ & Insufficient peak flows & No, insufficient peak flows. \\
\hline 3 & 04010510 & Grand Portage River at Grand Portage, Minn. & $06 / 29 / 1991$ & $11 / 18 / 1991$ & Insufficient peak flows & No, insufficient peak flows. \\
\hline 4 & 04010520 & Hollow Rock Creek near Red Rock, Minn. & $05 / 30 / 2003$ & $10 / 23 / 2004$ & Insufficient peak flows & No, insufficient peak flows. \\
\hline 5 & 04010528 & Reservation River near Grand Portage, Minn. & $05 / 30 / 2003$ & $05 / 31 / 2004$ & Insufficient peak flows & No, insufficient peak flows. \\
\hline 6 & 04010530 & Reservation River near Hovland, Minn. & $06 / 29 / 1991$ & $07 / 21 / 2011$ & $\begin{array}{l}\text { Peak flow(s) exceeded maximum recordable discharge. Modi- } \\
\text { fied perception thresholds on peak flow(s). Accepted modified } \\
\text { results from EMA }\end{array}$ & Yes. \\
\hline 7 & 04011370 & $\begin{array}{l}\text { Little Devil Track River near Grand Marais, } \\
\text { Minn. }\end{array}$ & 04/20/1961 & $04 / 24 / 1982$ & $\begin{array}{l}\text { Low outlier(s) flagged by Bulletin 17B method. } \\
\text { Accepted default results from EMA }\end{array}$ & Yes. \\
\hline 8 & 04011390 & $\begin{array}{l}\text { Little Devil Track River tributary near Grand } \\
\text { Marais, Minn. }\end{array}$ & 05/05/1966 & $10 / 23 / 1980$ & Accepted default results from Bulletin 17B & Yes. \\
\hline 9 & 04011990 & $\begin{array}{l}\text { Cascade River at Forest Road } 45 \text { near Grand } \\
\text { Marais, Minn. }\end{array}$ & 05/31/1985 & 05/01/2011 & Accepted default results from Bulletin 17B & Yes. \\
\hline 10 & 04012500 & Poplar River at Lutsen, Minn. & $04 / 25 / 1913$ & $05 / 22 / 1987$ & $\begin{array}{l}\text { Peak flow(s) exceeded high-outlier criterion. Insufficient evidence } \\
\text { to extend historical period. Accepted default results from } \\
\text { Bulletin 17B }\end{array}$ & Yes. \\
\hline 11 & 04013100 & $\begin{array}{l}\text { Lake Superior tributary near Taconite Harbor, } \\
\text { Minn. }\end{array}$ & 05/23/1964 & 04/13/1981 & Accepted default results from Bulletin 17B & Yes. \\
\hline 12 & 04013200 & Caribou River near Little Marais, Minn. & $04 / 20 / 1961$ & $05 / 31 / 1985$ & $\begin{array}{l}\text { Peak flow(s) exceeded high-outlier criterion. Matched historical } \\
\text { period of Baptism River (streamgage 04014500), which had } \\
\text { large peak in } 1977 \text { and no other larger peak flows. Accepted } \\
\text { modified results from Bulletin 17B }\end{array}$ & Yes. \\
\hline 13 & 04013400 & Little Marais River near Little Marais, Minn. & $11 / 07 / 2000$ & $10 / 18 / 2007$ & Insufficient peak flows & No, insufficient peak flows. \\
\hline 14 & 04014500 & Baptism River near Beaver Bay, Minn. & $05 / 08 / 1930$ & $07 / 04 / 1993$ & Accepted default results from Bulletin 17B & Yes. \\
\hline 15 & 04015070 & $\begin{array}{l}\text { Lake Superior tributary at Split Rock State Park, } \\
\text { Minn. }\end{array}$ & $11 / 07 / 2000$ & $10 / 19 / 2007$ & Insufficient peak flows & No, insufficient peak flows. \\
\hline 16 & 04015150 & Crow Creek near Silver Creek, Minn. & $04 / 13 / 1960$ & $04 / 23 / 1975$ & Accepted default results from Bulletin 17B & Yes. \\
\hline 17 & 04015200 & $\begin{array}{l}\text { Encampment River tributary at Silver Creek, } \\
\text { Minn. }\end{array}$ & $04 / 13 / 1960$ & $04 / 28 / 1990$ & Accepted default results from Bulletin 17B & Yes. \\
\hline 18 & 04015250 & Silver Creek tributary near Two Harbors, Minn. & 09/30/1965 & $10 / 26 / 2010$ & $\begin{array}{l}\text { Peak flow(s) at or below the minimum recordable discharge. } \\
\text { Accepted default results from EMA }\end{array}$ & Yes. \\
\hline 19 & 04015300 & Little Stewart River near Two Harbors, Minn. & $04 / 13 / 1960$ & $03 / 25 / 2000$ & Accepted default results from Bulletin 17B & Yes. \\
\hline 20 & 04015330 & Knife River near Two Harbors, Minn. & 04/23/1975 & $06 / 22 / 2011$ & Accepted default results from Bulletin 17B & Yes. \\
\hline
\end{tabular}


Table 1-1. Streamgages in and bordering Minnesota with peak-flow data through water year 2011._Continued

[Bulletin 17B method (Interagency Advisory Committee on Water Data, 1982). EMA, expected moments algorithm (Cohn and others, 1997); **/**, month and day of occurrence unknown]

\begin{tabular}{|c|c|c|c|c|c|c|}
\hline $\begin{array}{c}\text { Map } \\
\text { number } \\
\text { (fig. 1) }\end{array}$ & $\begin{array}{c}\text { Streamgage } \\
\text { number }\end{array}$ & Streamgage name & $\begin{array}{c}\text { Start of } \\
\text { systematic } \\
\text { record }\end{array}$ & $\begin{array}{c}\text { End of } \\
\text { systematic } \\
\text { record }\end{array}$ & Peak-flow analysis comments & $\begin{array}{l}\text { Weighting of independent } \\
\text { estimates (WIE) calculated }\end{array}$ \\
\hline 21 & 04015360 & $\begin{array}{l}\text { Lake Superior tributary number } 2 \text { at French River, } \\
\text { Minn. }\end{array}$ & 09/07/1964 & $04 / 23 / 1981$ & Accepted default results from Bulletin 17B & Yes. \\
\hline 22 & 04015370 & Talmadge River at Duluth, Minn. & $09 / 07 / 1964$ & $05 / 08 / 2000$ & Accepted default results from Bulletin 17B & Yes. \\
\hline 23 & 04015400 & Miller Creek at Duluth, Minn. & $05 / 20 / 1960$ & $09 / 03 / 1985$ & $\begin{array}{l}\text { Affected by urbanization, used station skew. Low outlier(s) } \\
\text { flagged by Bulletin 17B method. Accepted modified results } \\
\text { from EMA }\end{array}$ & No, station skew. \\
\hline 24 & 04015410 & Miller Creek near mouth at Duluth, Minn. & $06 / 24 / 1993$ & $06 / 24 / 1993$ & Insufficient peak flows & No, insufficient peak flows. \\
\hline 25 & 04015415 & $\begin{array}{l}\text { Lake Superior tributary at West 9th Street in } \\
\text { Duluth, Minn. }\end{array}$ & $04 / 23 / 2001$ & $06 / 22 / 2011$ & $\begin{array}{l}\text { Affected by urbanization, used station skew. Low outlier(s) } \\
\text { flagged by Bulletin 17B method. Accepted modified results } \\
\text { from Bulletin 17B (EMA confidence limits were erroneous) }\end{array}$ & No, station skew. \\
\hline 26 & 04015455 & South Branch Partridge River near Babbitt, Minn. & $09 / 24 / 1977$ & $04 / 19 / 1980$ & Insufficient peak flows & No, insufficient peak flows. \\
\hline 27 & 04015475 & $\begin{array}{l}\text { Partridge River above Colby Lake at Hoyt Lakes, } \\
\text { Minn. }\end{array}$ & $04 / 22 / 1979$ & 08/25/1988 & $\begin{array}{l}\text { Peak flow(s) exceeded high-outlier criterion. Insufficient evidence } \\
\text { to extend historical period. Accepted default results from } \\
\text { Bulletin 17B }\end{array}$ & Yes. \\
\hline 28 & 04015500 & Second Creek near Aurora, Minn. & $04 / 12 / 1955$ & $09 / 20 / 1980$ & Accepted default results from Bulletin 17B & Yes. \\
\hline 29 & 04016000 & Partridge River near Aurora, Minn. & $06 / 17 / 1943$ & $04 / 27 / 1982$ & Accepted default results from Bulletin 17B & Yes. \\
\hline 30 & 04016500 & St. Louis River near Aurora, Minn. & $06 / 16 / 1943$ & 07/05/1999 & Accepted default results from Bulletin 17B & Yes. \\
\hline 31 & 04017000 & Embarrass River at Embarrass, Minn. & $06 / 17 / 1943$ & $06 / 26 / 1964$ & Accepted default results from Bulletin 17B & Yes. \\
\hline 32 & 04017700 & McKinley Lake tributary at McKinley, Minn. & $04 / 13 / 1960$ & $06 / 28 / 1981$ & Accepted default results from Bulletin 17B & Yes. \\
\hline 33 & 04018000 & Embarrass River near McKinley, Minn. & $04 / 20 / 1954$ & $05 / 25 / 1962$ & Insufficient peak flows & No, insufficient peak flows. \\
\hline 34 & 04018750 & St. Louis River at Forbes, Minn. & 06/01/1964 & $04 / 25 / 1989$ & Accepted default results from Bulletin 17B & Yes. \\
\hline 35 & 04018800 & East Two River tributary at Virginia, Minn. & 06/11/1959 & 04/16/1972 & Accepted default results from Bulletin 17B & Yes. \\
\hline 36 & 04018900 & East Two River near Iron Junction, Minn. & 04/01/1967 & 04/19/1979 & Accepted default results from Bulletin 17B & Yes. \\
\hline 37 & 04019000 & West Two River near Iron Junction, Minn. & $04 / 17 / 1954$ & $04 / 21 / 1979$ & Accepted default results from Bulletin 17B & Yes. \\
\hline 38 & 04019300 & West Swan River near Silica, Minn. & $06 / 24 / 1964$ & 04/20/1979 & Accepted default results from Bulletin 17B & Yes. \\
\hline 39 & 04019500 & East Swan River near Toivola, Minn. & $04 / 13 / 1954$ & 04/13/1971 & Accepted default results from Bulletin 17B & Yes. \\
\hline 40 & 04020000 & Swan River near Toivola, Minn. & 07/04/1953 & $04 / 21 / 1961$ & Insufficient peak flows & No, insufficient peak flows. \\
\hline 41 & 04020480 & $\begin{array}{l}\text { North Branch Whiteface River near Fairbanks, } \\
\text { Minn. }\end{array}$ & 04/23/1979 & $04 / 10 / 2011$ & Accepted default results from Bulletin 17B & Yes. \\
\hline 42 & 04020700 & Bug Creek at Shaw, Minn. & 04/23/1979 & $04 / 10 / 2011$ & $\begin{array}{l}\text { Low outlier(s) flagged by Bulletin 17B method. Accepted default } \\
\text { results from EMA }\end{array}$ & Yes. \\
\hline 43 & 04021205 & Floodwood River above Floodwood, Minn. & 04/19/1972 & 07/20/1987 & Accepted default results from Bulletin 17B & Yes. \\
\hline 44 & 04021520 & $\begin{array}{l}\text { Stoney Brook at Pine Drive near Brookston, } \\
\text { Minn. }\end{array}$ & $05 / 28 / 2005$ & $10 / 28 / 2010$ & Insufficient peak flows & No, insufficient peak flows. \\
\hline 45 & 04021530 & Stoney Brook at Brookston, Minn. & 06/10/1984 & 06/10/1984 & Insufficient peak flows & No, insufficient peak flows. \\
\hline
\end{tabular}


Table 1-1. Streamgages in and bordering Minnesota with peak-flow data through water year 2011.-Continued

[Bulletin 17B method (Interagency Advisory Committee on Water Data, 1982). EMA, expected moments algorithm (Cohn and others, 1997); **/**, month and day of occurrence unknown]

\begin{tabular}{|c|c|c|c|c|c|c|}
\hline $\begin{array}{l}\text { Map } \\
\text { number } \\
\text { (fig. 1) }\end{array}$ & $\begin{array}{c}\text { Streamgage } \\
\text { number }\end{array}$ & Streamgage name & $\begin{array}{l}\text { Start of } \\
\text { systematic } \\
\text { record }\end{array}$ & $\begin{array}{l}\text { End of } \\
\text { systematic } \\
\text { record }\end{array}$ & Peak-flow analysis comments & $\begin{array}{l}\text { Weighting of independent } \\
\text { estimates (WIE) calculated? }\end{array}$ \\
\hline 46 & 04021690 & Cloquet River near Toimi, Minn. & $04 / 28 / 1986$ & $04 / 30 / 2011$ & $\begin{array}{l}\text { Peak flow(s) at or below the minimum recordable discharge. } \\
\text { Accepted modified results from EMA }\end{array}$ & Yes. \\
\hline 47 & 04023150 & Simian Creek near Brookston, Minn. & 06/11/1984 & $06 / 11 / 1984$ & Insufficient peak flows & No, insufficient peak flows. \\
\hline 48 & 04023600 & Squaw Creek near Cloquet, Minn. & 06/11/1984 & $06 / 11 / 1984$ & Insufficient peak flows & No, insufficient peak flows. \\
\hline 49 & 04024000 & St. Louis River at Scanlon, Minn. & $06 / 12 / 1908$ & $04 / 10 / 2011$ & $\begin{array}{l}\text { Low outlier(s) flagged by Bulletin 17B method. Accepted default } \\
\text { results from EMA }\end{array}$ & No, large drainage basin. \\
\hline 50 & 04024015 & Otter Creek near Cloquet, Minn. & 06/10/1984 & $06 / 10 / 1984$ & Insufficient peak flows & No, insufficient peak flows. \\
\hline 51 & 04024090 & Elim Creek near Holyoke, Minn. & 06/17/1976 & $08 / 23 / 1978$ & Insufficient peak flows & No, insufficient peak flows. \\
\hline 52 & 04024093 & $\begin{array}{l}\text { Skunk Creek below Elim Creek near Holyoke, } \\
\text { Minn. }\end{array}$ & 06/18/1976 & $06 / 15 / 1978$ & Insufficient peak flows & No, insufficient peak flows. \\
\hline 53 & 04024095 & Nemadji River near Holyoke, Minn. & 09/20/1972 & $08 / 03 / 2011$ & $\begin{array}{l}\text { Low outlier(s) flagged by Bulletin 17B method. Accepted default } \\
\text { results from EMA }\end{array}$ & Yes. \\
\hline 54 & 04024098 & Deer Creek near Holyoke, Minn. & 09/24/1977 & $04 / 22 / 2001$ & Accepted default results from Bulletin 17B & Yes. \\
\hline 55 & 04024100 & Rock Creek near Blackhoof, Minn. & 04/20/1961 & 06/01/1987 & $\begin{array}{l}\text { Low outlier(s) flagged by Bulletin 17B method. Peak flow(s) } \\
\text { exceeded maximum recordable discharge. Modified perception } \\
\text { thresholds on peak flow(s). Accepted modified results from } \\
\text { EMA }\end{array}$ & Yes. \\
\hline 56 & 04024110 & Rock Creek tributary near Blackhoof, Minn. & 04/20/1961 & $04 / 27 / 1986$ & Accepted default results from Bulletin 17B & No, small drainage basin. \\
\hline 57 & 04024200 & South Fork Nemadji River near Holyoke, Minn. & $05 / 14 / 1961$ & 09/03/1985 & Accepted default results from Bulletin 17B & Yes. \\
\hline 58 & 05030000 & Otter Tail River near Detroit Lakes, Minn. & $06 / 20 / 1937$ & $04 / 10 / 1971$ & Accepted default results from Bulletin 17B & Yes. \\
\hline 59 & 05030500 & Otter Tail River near Elizabeth, Minn. & 06/23/1904 & $06 / 01 / 2011$ & $\begin{array}{l}\text { Low outlier(s) flagged by Bulletin 17B method. Accepted default } \\
\text { results from EMA }\end{array}$ & Yes. \\
\hline 60 & 05040000 & Pelican River near Detroit Lakes, Minn. & $06 / 26 / 1943$ & $06 / 20 / 1953$ & Accepted default results from Bulletin 17B & Yes. \\
\hline 61 & 05040500 & Pelican River near Fergus Falls, Minn. & 10/11/1909 & $04 / 05 / 1980$ & Accepted default results from Bulletin 17B & Yes. \\
\hline 62 & 05046000 & $\begin{array}{l}\text { Otter Tail River below Orwell Dam near Fergus } \\
\text { Falls, Minn. }\end{array}$ & 05/18/1931 & $05 / 02 / 2011$ & $\begin{array}{l}\text { Affected by regulation, used station skew. Accepted modified } \\
\text { results from Bulletin 17B }\end{array}$ & No, station skew. \\
\hline 63 & 05046250 & Otter Tail River near Foxhome, Minn. & $06 / 14 / 1990$ & 06/05/1999 & Accepted default results from Bulletin 17B & No, large drainage basin. \\
\hline 64 & 05046475 & Otter Tail River diversion at Breckenridge, Minn. & $06 / 14 / 2005$ & $04 / 07 / 2011$ & Insufficient peak flows & No, insufficient peak flows. \\
\hline 65 & 05047500 & $\begin{array}{l}\text { Mustinka River above Twelvemile Creek near } \\
\text { Charlesville, Minn. }\end{array}$ & 06/05/1944 & $07 / 13 / 1955$ & $\begin{array}{l}\text { Peak flow(s) exceeded high-outlier criterion. The Mustinka River } \\
\text { above Wheaton (streamgage } 05049000 \text { ) had } 3 \text { peak flows in } \\
62 \text { years of record that equaled or exceeded the } 1952 \text { peak flow. } \\
\text { Unable to extend historical period. Accepted default results } \\
\text { from Bulletin 17B }\end{array}$ & Yes. \\
\hline 66 & 05047900 & $\begin{array}{l}\text { Twelvemile Creek at County Road 6, near Du- } \\
\text { mont, Minn. }\end{array}$ & 05/18/1996 & $04 / 04 / 2011$ & $\begin{array}{l}\text { Low outlier(s) flagged by Bulletin 17B method. Accepted default } \\
\text { results from EMA }\end{array}$ & Yes. \\
\hline
\end{tabular}


Table 1-1. Streamgages in and bordering Minnesota with peak-flow data through water year 2011._Continued

[Bulletin 17B method (Interagency Advisory Committee on Water Data, 1982). EMA, expected moments algorithm (Cohn and others, 1997); **/**, month and day of occurrence unknown]

\begin{tabular}{|c|c|c|c|c|c|c|}
\hline $\begin{array}{c}\text { Map } \\
\text { number } \\
\text { (fig. 1) }\end{array}$ & $\begin{array}{c}\text { Streamgage } \\
\text { number }\end{array}$ & Streamgage name & $\begin{array}{l}\text { Start of } \\
\text { systematic } \\
\text { record }\end{array}$ & $\begin{array}{l}\text { End of } \\
\text { systematic } \\
\text { record }\end{array}$ & Peak-flow analysis comments & $\begin{array}{r}\text { Weighting of } i \\
\text { estimates (WIE }\end{array}$ \\
\hline 67 & 05047960 & $\begin{array}{l}\text { West Branch Twelvemile Creek near Graceville, } \\
\text { Minn. }\end{array}$ & $04 / 13 / 1964$ & $5 / 21 / 1972$ & Insufficient peak flows & No, insufficient $p$ \\
\hline 68 & 05047970 & $\begin{array}{l}\text { West Branch Twelvemile Creek tributary near } \\
\text { Graceville, Minn. }\end{array}$ & $04 / 13 / 1964$ & $03 / 26 / 1989$ & Accepted default results from Bulletin 17B & Yes. \\
\hline 69 & 05048000 & $\begin{array}{l}\text { Mustinka River below Twelvemile Creek near } \\
\text { Charlesville, Minn. }\end{array}$ & 06/04/1944 & 07/13/1955 & $\begin{array}{l}\text { Low outlier(s) flagged by Bulletin 17B method. Accepted default } \\
\text { results from EMA }\end{array}$ & Yes. \\
\hline 70 & 05048500 & $\begin{array}{l}\text { Mustinka River, old channel, at Twelvemile Creek } \\
\text { mouth, Minn. }\end{array}$ & 06/04/1944 & $04 / 01 / 1955$ & $\begin{array}{l}\text { Peak flow(s) exceeded high-outlier criterion. The Mustinka River } \\
\text { above Wheaton (streamgage 05049000) had } 3 \text { peak flows in } \\
62 \text { years of record that equaled or exceeded the } 1952 \text { peak flow. } \\
\text { Unable to extend historical period. Accepted default results } \\
\text { from Bulletin 17B }\end{array}$ & $\begin{array}{l}\text { No, regression no } \\
\text { old channel. }\end{array}$ \\
\hline 71 & 05049000 & Mustinka River above Wheaton, Minn. & $* * / * * / 1916$ & $04 / 06 / 2011$ & $\begin{array}{l}\text { Low outlier(s) flagged by Bulletin 17B method. Accepted default } \\
\text { results from EMA }\end{array}$ & Yes. \\
\hline 72 & 05049200 & Eighteen Mile Creek near Wheaton, Minn. & 06/01/1965 & $05 / 18 / 1996$ & Accepted default results from Bulletin 17B & Yes. \\
\hline 73 & 05050000 & Bois de Sioux River near White Rock, S. Dak. & 07/11/1942 & $04 / 15 / 2011$ & $\begin{array}{l}\text { Affected by regulation, used station skew. Accepted modified } \\
\text { results from Bulletin 17B }\end{array}$ & No, station skew. \\
\hline 74 & 05050700 & Rabbit River near Nashua, Minn. & $04 / 12 / 1979$ & $03 / 31 / 2006$ & $\begin{array}{l}\text { Low outlier(s) flagged by Bulletin 17B method. Used a fixed low- } \\
\text { outlier threshold. Accepted modified results from EMA }\end{array}$ & Yes. \\
\hline 75 & 05051000 & Rabbit River at Campbell, Minn. & 04/03/1943 & $04 / 01 / 1952$ & $\begin{array}{l}\text { Peak flow(s) exceeded high-outlier criterion. The Mustinka River } \\
\text { above Wheaton (streamgage 05049000) had } 3 \text { peak flows in } \\
62 \text { years of record that equaled or exceeded the } 1952 \text { peak flow. } \\
\text { Unable to extend historical period. Accepted default results } \\
\text { from Bulletin 17B }\end{array}$ & Yes. \\
\hline 76 & 05051300 & Bois de Sioux River near Doran, Minn. & 03/16/1990 & 04/07/2011 & $\begin{array}{l}\text { Affected by regulation, used station skew. Low outlier(s) flagged } \\
\text { by Bulletin 17B method. Accepted modified results from EMA }\end{array}$ & No, station skew. \\
\hline 77 & 05051500 & Red River of the North at Wahpeton, N. Dak. & $06 / 07 / 1942$ & $04 / 07 / 2011$ & $\begin{array}{l}\text { Historical peak flow(s) detected. Extended historical period back } \\
\text { to } 1897 \text { to include } 1897 \text { peak. Accepted default results from } \\
\text { EMA }\end{array}$ & No, station skew. \\
\hline 78 & 05051522 & Red River of the North at Hickson, N. Dak. & $03 / 31 / 1976$ & $04 / 07 / 2011$ & $\begin{array}{l}\text { Affected by regulation, used station skew. Accepted modified } \\
\text { results from EMA }\end{array}$ & No, station skew. \\
\hline 79 & 05054000 & Red River of the North at Fargo, N. Dak. & 04/07/1897 & 04/09/2011 & $\begin{array}{l}\text { Historical peak flow(s) detected. Extended historical period back } \\
\text { to } 1897 \text { to include } 1897 \text { peak. Accepted default results from } \\
\text { EMA }\end{array}$ & No, station skew. \\
\hline 82 & 05061200 & Whiskey Creek at Barnesville, Minn. & 03/13/1961 & $04 / 02 / 2011$ & Accepted default results from Bulletin 17B & Yes. \\
\hline
\end{tabular}


Table 1-1. Streamgages in and bordering Minnesota with peak-flow data through water year 2011.-Continued

[Bulletin 17B method (Interagency Advisory Committee on Water Data, 1982). EMA, expected moments algorithm (Cohn and others, 1997); **/**, month and day of occurrence unknown]

\begin{tabular}{|c|c|c|c|c|c|c|}
\hline $\begin{array}{l}\text { Map } \\
\text { number } \\
\text { (fig. 1) }\end{array}$ & $\begin{array}{l}\text { Streamgage } \\
\text { number }\end{array}$ & Streamgage name & $\begin{array}{l}\text { Start of } \\
\text { systematic } \\
\text { record }\end{array}$ & $\begin{array}{l}\text { End of } \\
\text { systematic } \\
\text { record }\end{array}$ & Peak-flow analysis comments & $\begin{array}{l}\text { Weighting of independent } \\
\text { estimates (WIE) calculated? }\end{array}$ \\
\hline 83 & 05061400 & Spring Creek above Downer, Minn. & 03/03/1961 & $06 / 20 / 2000$ & Accepted default results from Bulletin 17B & Yes. \\
\hline 84 & 05061500 & South Branch Buffalo River at Sabin, Minn. & $03 / 18 / 1945$ & $04 / 04 / 2011$ & Accepted default results from Bulletin 17B & Yes. \\
\hline 85 & 05062000 & Buffalo River near Dilworth, Minn. & $04 / 10 / 1931$ & $04 / 06 / 2011$ & $\begin{array}{l}\text { Low outlier(s) flagged by Bulletin 17B method. Accepted default } \\
\text { results from EMA }\end{array}$ & Yes. \\
\hline 86 & 05062280 & Mosquito Creek near Bagley, Minn. & $04 / 18 / 1961$ & $03 / 24 / 1985$ & Accepted default results from Bulletin 17B & Yes. \\
\hline 87 & 05062470 & Marsh Creek tributary near Mahnomen, Minn. & $03 / 17 / 1961$ & $05 / 12 / 1985$ & $\begin{array}{l}\text { Low outlier(s) flagged by Bulletin 17B method. Accepted default } \\
\text { results from EMA }\end{array}$ & Yes. \\
\hline 88 & 05062500 & Wild Rice River at Twin Valley, Minn. & 07/22/1909 & $04 / 11 / 2011$ & Accepted default results from Bulletin 17B & Yes. \\
\hline 89 & 05062700 & Wild Rice River tributary near Twin Valley, Minn. & $05 / 14 / 1961$ & $05 / 12 / 1985$ & $\begin{array}{l}\text { Low outlier(s) flagged by Bulletin 17B method. Accepted default } \\
\text { results from EMA }\end{array}$ & Yes. \\
\hline 90 & 05062800 & Coon Creek near Twin Valley, Minn. & 06/08/1962 & $06 / 09 / 1984$ & Accepted default results from Bulletin 17B & Yes. \\
\hline 91 & 05062850 & Coon Creek tributary near Twin Valley, Minn. & $06 / 20 / 2000$ & $04 / 08 / 2001$ & Insufficient peak flows & No, insufficient peak flows. \\
\hline 92 & 05062900 & Wild Rice River above Ada, Minn. & $05 / 14 / 1985$ & $04 / 01 / 1990$ & Insufficient peak flows & No, insufficient peak flows. \\
\hline 93 & 05063000 & Wild Rice River near Ada, Minn. & 04/09/1948 & $07 / 04 / 1953$ & Insufficient peak flows & No, insufficient peak flows. \\
\hline 94 & 05063200 & Spring Creek tributary near Ogema, Minn. & $06 / 02 / 1963$ & 04/03/1989 & $\begin{array}{l}\text { Low outlier(s) flagged by Bulletin 17B method. Accepted default } \\
\text { results from EMA }\end{array}$ & Yes. \\
\hline 95 & 05063398 & $\begin{array}{l}\text { South Branch Wild Rice River at County Road } 27 \\
\text { near Felton, Minn. }\end{array}$ & $10 / 30 / 2004$ & $04 / 11 / 2011$ & Insufficient peak flows & No, insufficient peak flows. \\
\hline 96 & 05063500 & South Branch Wild Rice River near Borup, Minn. & $07 / 12 / 1944$ & $06 / 11 / 1984$ & $\begin{array}{l}\text { Low outlier(s) flagged by Bulletin 17B method. Accepted default } \\
\text { results from EMA }\end{array}$ & Yes. \\
\hline 97 & 05063850 & State Ditch 45 tributary near Ulen, Minn. & $06 / 09 / 2002$ & $04 / 04 / 2011$ & $\begin{array}{l}\text { Low outlier(s) flagged by Bulletin 17B method. Peak flow(s) at } \\
\text { or below the minimum recordable discharge. Accepted default } \\
\text { results from EMA }\end{array}$ & Yes. \\
\hline 98 & 05064000 & Wild Rice River at Hendrum, Minn. & $07 / 15 / 1944$ & $04 / 07 / 2011$ & $\begin{array}{l}\text { Low outlier(s) flagged by Bulletin 17B method. Accepted default } \\
\text { results from EMA }\end{array}$ & Yes. \\
\hline 99 & 05064500 & Red River of the North at Halstad, Minn. & $04 / 15 / 1936$ & $04 / 12 / 2011$ & $\begin{array}{l}\text { Affected by regulation, used station skew. Accepted modified } \\
\text { results from Bulletin 17B }\end{array}$ & No, station skew. \\
\hline 100 & 05067000 & Marsh River below Ada, Minn. & $04 / 16 / 1948$ & 03/05/1973 & Insufficient peak flows & No, insufficient peak flows. \\
\hline 101 & 05067050 & Marsh River Ditch near Ada, Minn. & 05/13/1985 & 04/10/2011 & River/stream is part of unnatural diversion channel. No analysis & No analysis. \\
\hline 102 & 05067500 & Marsh River near Shelly, Minn. & 07/11/1944 & $04 / 09 / 2011$ & $\begin{array}{l}\text { Low outlier(s) flagged by Bulletin 17B method. Used a fixed low- } \\
\text { outlier threshold. Accepted modified results from EMA }\end{array}$ & Yes. \\
\hline 103 & 05068000 & Sand Hill River at Beltrami, Minn. & $04 / 05 / 1943$ & $07 / 05 / 1958$ & $\begin{array}{l}\text { Affected by regulation, used station skew. Accepted modified } \\
\text { results from Bulletin 17B }\end{array}$ & No, station skew. \\
\hline 104 & 05069000 & Sand Hill River at Climax, Minn. & $04 / 07 / 1943$ & $04 / 10 / 2011$ & Accepted default results from Bulletin 17B & Yes. \\
\hline 105 & 05070000 & Red River of the North near Thompson, N. Dak. & 03/31/1999 & $04 / 13 / 2011$ & $\begin{array}{l}\text { Affected by regulation, used station skew. Accepted modified } \\
\text { results from Bulletin 17B }\end{array}$ & No, station skew. \\
\hline
\end{tabular}


Table 1-1. Streamgages in and bordering Minnesota with peak-flow data through water year 2011._Continued

[Bulletin 17B method (Interagency Advisory Committee on Water Data, 1982). EMA, expected moments algorithm (Cohn and others, 1997); **/**, month and day of occurrence unknown]

\begin{tabular}{|c|c|c|c|c|c|c|}
\hline $\begin{array}{c}\text { Map } \\
\text { number } \\
\text { (fig. 1) }\end{array}$ & $\begin{array}{l}\text { Streamgage } \\
\text { number }\end{array}$ & Streamgage name & $\begin{array}{l}\text { Start of } \\
\text { systematic } \\
\text { record }\end{array}$ & $\begin{array}{l}\text { End of } \\
\text { systematic } \\
\text { record }\end{array}$ & Peak-flow analysis comments & $\begin{array}{l}\text { Weighting of independent } \\
\text { estimates (WIE) calculated? }\end{array}$ \\
\hline 106 & 05073600 & South Branch Battle River at Northome, Minn. & $05 / 29 / 1960$ & $08 / 17 / 1985$ & $\begin{array}{l}\text { Low outlier(s) flagged by Bulletin 17B method. Accepted default } \\
\text { results from EMA }\end{array}$ & No, small drainage basin. \\
\hline 107 & 05073750 & Spring Creek near Blackduck, Minn. & $04 / 13 / 1960$ & $04 / 23 / 1985$ & $\begin{array}{l}\text { Peak flow(s) exceeded high-outlier criterion. Insufficient evidence } \\
\text { to extend historical period. Peak flow(s) exceeded maximum } \\
\text { recordable discharge. Modified perception thresholds on peak } \\
\text { flow(s). Accepted modified results from EMA }\end{array}$ & Yes. \\
\hline 108 & 05073800 & Perry Creek near Shooks, Minn. & $07 / 16 / 1960$ & $06 / 26 / 1985$ & Accepted default results from Bulletin 17B & No, small drainage basin. \\
\hline 109 & 05073870 & Mud River at Redby, Minn. & 04/29/1986 & $04 / 29 / 1986$ & Insufficient peak flows & No, insufficient peak flows. \\
\hline 110 & 05073900 & Pike Creek at Lower Red Lake, Minn. & 04/30/1986 & $04 / 30 / 1986$ & Insufficient peak flows & No, insufficient peak flows. \\
\hline 111 & 05073980 & Sandy River near Red Lake, Minn. & 04/02/1986 & $04 / 02 / 1986$ & Insufficient peak flows & No, insufficient peak flows. \\
\hline 112 & 05074500 & Red Lake River near Red Lake, Minn. & $07 / 04 / 1933$ & $09 / 29 / 2011$ & $\begin{array}{l}\text { Affected by regulation, used station skew. Peak flow(s) exceeded } \\
\text { maximum recordable discharge. Modified perception thresholds } \\
\text { on peak flow(s). Accepted modified results from EMA }\end{array}$ & No, station skew. \\
\hline 113 & 05075000 & $\begin{array}{l}\text { Red Lake River at High Landing near Goodridge, } \\
\text { Minn. }\end{array}$ & $04 / 05 / 1930$ & $06 / 08 / 2011$ & $\begin{array}{l}\text { Affected by regulation, used station skew. Low outlier(s) flagged } \\
\text { by Bulletin 17B method. Used a fixed low-outlier threshold. } \\
\text { Accepted modified results from EMA }\end{array}$ & No, station skew. \\
\hline 114 & 05075500 & Thief River near Gatzke, Minn. & 06/07/1954 & $07 / 05 / 1956$ & Insufficient peak flows & No, insufficient peak flows. \\
\hline 115 & 05075700 & Mud River near Grygla, Minn. & $04 / 26 / 1979$ & $04 / 11 / 2011$ & $\begin{array}{l}\text { Low outlier(s) flagged by Bulletin 17B method. Accepted default } \\
\text { results from EMA }\end{array}$ & Yes. \\
\hline 116 & 05076000 & Thief River near Thief River Falls, Minn. & 07/19/1909 & $04 / 06 / 2011$ & $\begin{array}{l}\text { Low outlier(s) flagged by Bulletin 17B method. Accepted default } \\
\text { results from EMA }\end{array}$ & Yes. \\
\hline 117 & 05076500 & Red Lake River at Thief River Falls, Minn. & $03 / 24 / 1910$ & 05/13/1930 & Accepted default results from Bulletin 17B & No, large drainage basin. \\
\hline 118 & 05076600 & $\begin{array}{l}\text { Red Lake River tributary near Thief River Falls, } \\
\text { Minn. }\end{array}$ & $06 / 08 / 1962$ & $09 / 07 / 1981$ & Accepted default results from Bulletin 17B & No, small drainage basin \\
\hline 119 & 05076690 & Clearwater River tributary near Bagley, Minn. & $04 / 07 / 2001$ & $04 / 01 / 2007$ & Insufficient peak flows & No, insufficient peak flows. \\
\hline 120 & 05077000 & Clearwater River near Pinewood, Minn. & $04 / 13 / 1940$ & 04/17/1972 & Insufficient peak flows & No, insufficient peak flows. \\
\hline 121 & 05077500 & Clearwater River near Leonard, Minn. & $05 / 15 / 1935$ & $04 / 21 / 1947$ & $\begin{array}{l}\text { Affected by regulation, used station skew. Accepted modified } \\
\text { results from Bulletin 17B }\end{array}$ & No, station skew. \\
\hline 122 & 05077600 & Clearwater River near Clearbrook, Minn. & $04 / 02 / 1986$ & $04 / 02 / 1986$ & Insufficient peak flows & No, insufficient peak flows. \\
\hline 123 & 05077700 & Ruffy Brook near Gonvick, Minn. & $04 / 20 / 1961$ & $04 / 11 / 2011$ & $\begin{array}{l}\text { Low outlier(s) flagged by Bulletin 17B method. Peak flow(s) at } \\
\text { or below the minimum recordable discharge. Accepted default } \\
\text { results from EMA }\end{array}$ & Yes. \\
\hline 124 & 05077850 & Clearwater River near Trail, Minn. & 03/30/1986 & 03/30/1986 & Insufficient peak flows & No, insufficient peak flows. \\
\hline 125 & 05078000 & Clearwater River at Plummer, Minn. & $04 / 27 / 1939$ & $06 / 29 / 2011$ & Accepted default results from Bulletin 17B & Yes. \\
\hline 126 & 05078100 & Lost River at Gonvick, Minn. & $04 / 26 / 1960$ & $04 / 13 / 1972$ & $\begin{array}{l}\text { Low outlier(s) flagged by Bulletin 17B method. Accepted default } \\
\text { results from EMA }\end{array}$ & Yes. \\
\hline
\end{tabular}


Table 1-1. Streamgages in and bordering Minnesota with peak-flow data through water year 2011._- Continued

[Bulletin 17B method (Interagency Advisory Committee on Water Data, 1982). EMA, expected moments algorithm (Cohn and others, 1997); **/**, month and day of occurrence unknown]

\begin{tabular}{|c|c|c|c|c|c|c|}
\hline $\begin{array}{l}\text { Map } \\
\text { number } \\
\text { (fig. 1) }\end{array}$ & $\begin{array}{c}\text { Streamgage } \\
\text { number }\end{array}$ & Streamgage name & $\begin{array}{l}\text { Start of } \\
\text { systematic } \\
\text { record }\end{array}$ & $\begin{array}{l}\text { End of } \\
\text { systematic } \\
\text { record }\end{array}$ & Peak-flow analysis comments & $\begin{array}{l}\text { Weighting of independent } \\
\text { estimates (WIE) calculated? }\end{array}$ \\
\hline 127 & 05078180 & Silver Creek near Clearbrook, Minn. & $04 / 06 / 1960$ & $05 / 11 / 1985$ & $\begin{array}{l}\text { Low outlier(s) flagged by Bulletin 17B method. Accepted default } \\
\text { results from EMA }\end{array}$ & Yes. \\
\hline 128 & 05078200 & Silver Creek tributary at Clearbrook, Minn. & $04 / 06 / 1960$ & $06 / 27 / 1981$ & Accepted default results from Bulletin 17B & Yes. \\
\hline 129 & 05078230 & Lost River at Oklee, Minn. & $04 / 23 / 1961$ & $06 / 28 / 2011$ & $\begin{array}{l}\text { Low outlier(s) flagged by Bulletin 17B method. Used a fixed low- } \\
\text { outlier threshold. Accepted modified results from EMA }\end{array}$ & Yes. \\
\hline 130 & 05078400 & Clearwater River tributary near Plummer, Minn. & $03 / 24 / 1961$ & 03/13/1990 & $\begin{array}{l}\text { Low outlier(s) flagged by Bulletin 17B method. Used a fixed low- } \\
\text { outlier threshold. Accepted modified results from EMA }\end{array}$ & Yes. \\
\hline 131 & 05078470 & Judicial Ditch 64 near Mentor, Minn. (SW4) & $06 / 10 / 2003$ & $03 / 13 / 2007$ & Insufficient peak flows & No, insufficient peak flows. \\
\hline 132 & 05078500 & Clearwater River at Red Lake Falls, Minn. & $03 / 22 / 1910$ & $06 / 29 / 2011$ & $\begin{array}{l}\text { Historical peak flow(s) detected. Extended historical period back } \\
\text { to } 1910 \text { and forward to } 1935 \text { to include } 1919 \text { peak flow. } \\
\text { Accepted modified results from EMA }\end{array}$ & Yes. \\
\hline 133 & 05078520 & Cyr Creek near Marcoux Corners, Minn. (SW5) & $06 / 23 / 2003$ & $10 / 30 / 2004$ & Insufficient peak flows & No, insufficient peak flows. \\
\hline 134 & 05078720 & $\begin{array}{l}\text { County Ditch } 140 \text { above Burnham-6 impound- } \\
\text { ment near Tilden, Minn. (SW8) }\end{array}$ & $10 / 30 / 2004$ & $05 / 26 / 2009$ & Insufficient peak flows & No, insufficient peak flows. \\
\hline 135 & 05078730 & County Ditch 140 near Benoit, Minn. (SW1) & $06 / 25 / 2003$ & $03 / 15 / 2007$ & Insufficient peak flows & No, insufficient peak flows. \\
\hline 136 & 05078770 & $\begin{array}{l}\text { Judicial Ditch } 66 \text { near Marcoux Corners, Minn. } \\
\text { (SW6) }\end{array}$ & $06 / 22 / 2003$ & $03 / 26 / 2009$ & Insufficient peak flows & No, insufficient peak flows. \\
\hline 137 & 05079000 & Red Lake River at Crookston, Minn. & $04 / 11 / 1897$ & $04 / 12 / 2011$ & $\begin{array}{l}\text { Historical peak flow(s) detected. Extended historical period back } \\
\text { to } 1897 \text { to include } 1897 \text { peak flow. Accepted modified results } \\
\text { from EMA }\end{array}$ & No, large drainage basin. \\
\hline 138 & 05079200 & $\begin{array}{l}\text { County Ditch } 72 \text { (Burnham Creek) near Maple } \\
\text { Bay, Minn. (SW3) }\end{array}$ & $06 / 25 / 2003$ & $06 / 15 / 2007$ & Insufficient peak flows & No, insufficient peak flows. \\
\hline 139 & 05079250 & County Ditch 65 near Maple Bay, Minn. (SW2) & $06 / 25 / 2003$ & $03 / 13 / 2007$ & Insufficient peak flows & No, insufficient peak flows. \\
\hline 140 & 05079901 & Burnham Creek near Crookston, Minn. & 03/29/1986 & $04 / 12 / 2011$ & Accepted default results from Bulletin 17B & Yes. \\
\hline 141 & 05080000 & Red Lake River at Fisher, Minn. & $06 / 24 / 2000$ & $04 / 13 / 2011$ & River/stream similar to streamgage 05079000 . No analysis & No, large drainage basin. \\
\hline 142 & 05082500 & Red River of the North at Grand Forks, N. Dak. & $04 / 18 / 1882$ & $04 / 14 / 2011$ & $\begin{array}{l}\text { Affected by regulation, used station skew. Accepted modified } \\
\text { results from EMA. Peak flows prior to } 1941 \text { have no affect due } \\
\text { to regulation }\end{array}$ & No, station skew. \\
\hline 143 & 05083500 & Red River of the North at Oslo, Minn. & $04 / 18 / 1936$ & $04 / 14 / 2011$ & $\begin{array}{l}\text { Affected by regulation, used station skew. Accepted modified } \\
\text { results from EMA }\end{array}$ & No, station skew. \\
\hline 144 & 05085420 & Snake River above Radium, Minn. & $06 / 12 / 2005$ & $06 / 06 / 2008$ & Insufficient peak flows & No, insufficient peak flows. \\
\hline 145 & 05085450 & Snake River above Warren, Minn. & $03 / 24 / 2009$ & $04 / 11 / 2011$ & Insufficient peak flows & No, insufficient peak flows. \\
\hline 146 & 05085500 & Snake River at Warren, Minn. & $03 / 28 / 1945$ & $04 / 21 / 1956$ & Insufficient peak flows & No, insufficient peak flows. \\
\hline 147 & 05085900 & Snake River above Alvarado, Minn. & $09 / 03 / 1993$ & $04 / 21 / 1996$ & Insufficient peak flows & No, insufficient peak flows. \\
\hline 148 & 05086000 & Snake River at Alvarado, Minn. & 03/30/1945 & $04 / 25 / 1956$ & Insufficient peak flows & No, insufficient peak flows. \\
\hline
\end{tabular}


[Bulletin 17B method (Interagency Advisory Committee on Water Data, 1982). EMA, expected moments algorithm (Cohn and others, 1997); **/**, month and day of occurrence unknown]

\begin{tabular}{|c|c|c|c|c|c|c|}
\hline $\begin{array}{c}\text { Map } \\
\text { number } \\
\text { (fig. 1) }\end{array}$ & $\begin{array}{l}\text { Streamgage } \\
\text { number }\end{array}$ & Streamgage name & $\begin{array}{l}\text { Start of } \\
\text { systematic } \\
\text { record }\end{array}$ & $\begin{array}{l}\text { End of } \\
\text { systematic } \\
\text { record }\end{array}$ & Peak-flow analysis comments & $\begin{array}{l}\text { Weighting of independent } \\
\text { estimates (WIE) calculated? }\end{array}$ \\
\hline 149 & 05086500 & $\begin{array}{l}\text { Snake River above Middle River near Big Woods, } \\
\text { Minn. }\end{array}$ & $03 / 31 / 1945$ & $03 / 31 / 1945$ & Insufficient peak flows & No, insufficient peak flows. \\
\hline 150 & 05086900 & Middle River near Newfolden, Minn. & $04 / 25 / 1979$ & $04 / 03 / 2011$ & Accepted default results from Bulletin 17B & Yes. \\
\hline 151 & 05087000 & Middle River near Strandquist, Minn. & $04 / 11 / 1954$ & 07/09/1956 & Insufficient peak flows & No, insufficient peak flows. \\
\hline 152 & 05087500 & Middle River at Argyle, Minn. & $03 / 30 / 1945$ & $04 / 05 / 2011$ & $\begin{array}{l}\text { Historical peak flow(s) detected. Extended historical period back } \\
\text { to } 1945 \text { to include } 1945 \text { peak flow. Low outlier(s) flagged by } \\
\text { Bulletin 17B method. Accepted modified results from EMA }\end{array}$ & Yes. \\
\hline 153 & 05090500 & Tamarac River near Strandquist, Minn. & 06/17/1954 & $07 / 09 / 1956$ & Insufficient peak flows & No, insufficient peak flows. \\
\hline 154 & 05091000 & Tamarac River at Stephen, Minn. & $03 / 29 / 1945$ & $03 / 29 / 1945$ & Insufficient peak flows & No, insufficient peak flows. \\
\hline 155 & 05091500 & Tamarac River near Stephen, Minn. & $03 / 29 / 1945$ & $04 / 09 / 1955$ & Insufficient peak flows & No, insufficient peak flows. \\
\hline 156 & 05092000 & Red River of the North at Drayton, N. Dak. & $04 / 19 / 1936$ & $04 / 19 / 2011$ & $\begin{array}{l}\text { Affected by regulation, used station skew. Accepted modified } \\
\text { results from EMA }\end{array}$ & No, station skew. \\
\hline 157 & 05092500 & Middle Branch Two Rivers near Hallock, Minn. & $04 / 19 / 1932$ & $04 / 29 / 1938$ & Insufficient peak flows & No, insufficient peak flows. \\
\hline 158 & 05093000 & South Branch Two Rivers at Pelan, Minn. & $03 / 21 / 1929$ & $07 / 10 / 1956$ & Accepted default results from Bulletin 17B & Yes. \\
\hline 159 & 05094000 & South Branch Two Rivers at Lake Bronson, Minn. & 03/20/1929 & $04 / 11 / 2011$ & $\begin{array}{l}\text { Low outlier(s) flagged by Bulletin 17B method. Accepted default } \\
\text { results from EMA }\end{array}$ & Yes. \\
\hline 160 & 05095000 & Two Rivers at Hallock, Minn. & $04 / 08 / 1912$ & $04 / 08 / 1943$ & Insufficient peak flows & No, insufficient peak flows. \\
\hline 161 & 05095500 & Two Rivers below Hallock, Minn. & $03 / 28 / 1945$ & $06 / 13 / 1955$ & Accepted default results from Bulletin 17B & Yes. \\
\hline 162 & 05096000 & North Branch Two Rivers near Lancaster, Minn. & $05 / 12 / 1930$ & $04 / 22 / 1955$ & Accepted default results from Bulletin 17B & Yes. \\
\hline 163 & 05096500 & State Ditch Number 85 near Lancaster, Minn. & 04/08/1929 & $04 / 24 / 1955$ & $\begin{array}{l}\text { Peak flow(s) exceeded high-outlier criterion. Insufficient evidence } \\
\text { to extend historical period. Low outlier(s) flagged by Bulletin } \\
\text { 17B method. Accepted default results from EMA }\end{array}$ & Yes. \\
\hline 164 & 05097000 & North Branch Two Rivers at Lancaster, Minn. & 09/26/1941 & $04 / 21 / 1956$ & Insufficient peak flows & No, insufficient peak flows. \\
\hline 165 & 05097500 & North Branch Two Rivers near Northcote, Minn. & 04/08/1941 & $04 / 18 / 1951$ & Insufficient peak flows & No, insufficient peak flows. \\
\hline 166 & 05102490 & Red River of the North at Pembina, N. Dak. & 03/27/1985 & $04 / 23 / 2011$ & $\begin{array}{l}\text { Drainage area too large to use regional skew, used station skew. } \\
\text { Accepted modified results from Bulletin 17B }\end{array}$ & No, large drainage basin. \\
\hline 167 & 05102500 & $\begin{array}{l}\text { Red River of the North at Emerson, Manitoba, } \\
\text { Canada }\end{array}$ & 04/11/1913 & $04 / 21 / 2011$ & $\begin{array}{l}\text { Affected by regulation, used station skew. Accepted modified } \\
\text { results from Bulletin 17B }\end{array}$ & No, station skew. \\
\hline 168 & 05103000 & Roseau River near Malung, Minn. & 04/06/1929 & $03 / 25 / 1946$ & Accepted default results from Bulletin 17B & Yes. \\
\hline 169 & 05104000 & South Fork Roseau River near Malung, Minn. & 04/07/1912 & $03 / 24 / 1946$ & Accepted default results from Bulletin 17B & Yes. \\
\hline 170 & 05104500 & $\begin{array}{l}\text { Roseau River below South Fork near Malung, } \\
\text { Minn. }\end{array}$ & 04/07/1929 & $04 / 13 / 2011$ & $\begin{array}{l}\text { Low outlier(s) flagged by Bulletin 17B method. Accepted default } \\
\text { results from EMA }\end{array}$ & Yes. \\
\hline 171 & 05106000 & Sprague Creek near Sprague, Manitoba, Canada & 05/30/1929 & $04 / 12 / 2011$ & $\begin{array}{l}\text { Low outlier(s) flagged by Bulletin 17B method. Accepted default } \\
\text { results from EMA }\end{array}$ & Yes. \\
\hline 172 & 05107000 & Pine Creek near Pine Creek, Minn. & 04/08/1929 & $05 / 13 / 1953$ & Accepted default results from Bulletin 17B & Yes. \\
\hline
\end{tabular}


Table 1-1. Streamgages in and bordering Minnesota with peak-flow data through water year 2011._- Continued

[Bulletin 17B method (Interagency Advisory Committee on Water Data, 1982). EMA, expected moments algorithm (Cohn and others, 1997); **/**, month and day of occurrence unknown]

\begin{tabular}{|c|c|c|c|c|c|c|}
\hline $\begin{array}{c}\text { Map } \\
\text { number } \\
\text { (fig. 1) }\end{array}$ & $\begin{array}{c}\text { Streamgage } \\
\text { number }\end{array}$ & Streamgage name & $\begin{array}{c}\text { Start of } \\
\text { systematic } \\
\text { record }\end{array}$ & $\begin{array}{l}\text { End of } \\
\text { systematic } \\
\text { record }\end{array}$ & Peak-flow analysis comments & $\begin{array}{l}\text { Weighting of independent } \\
\text { estimates (WIE) calculated? }\end{array}$ \\
\hline 173 & 05107500 & Roseau River at Ross, Minn. & 07/01/1919 & $04 / 17 / 2011$ & $\begin{array}{l}\text { Historical peak flow(s) detected. Extended historical period back } \\
\text { to } 1919 \text { to include } 1919 \text { and } 1927 \text { peak. Peak flow(s) exceeded } \\
\text { high-outlier criterion. Accepted modified results from EMA }\end{array}$ & Yes. \\
\hline 174 & 05109000 & Badger Creek near Badger, Minn. & 04/07/1929 & 05/10/1938 & Insufficient peak flows & No, insufficient peak flows. \\
\hline 175 & 05112000 & $\begin{array}{l}\text { Roseau River below State Ditch } 51 \text { near Caribou, } \\
\text { Minn. }\end{array}$ & 04/17/1917 & $04 / 25 / 2011$ & $\begin{array}{l}\text { Historical peak flow(s) detected. Effect of historical peak is negli- } \\
\text { gible, no historical period modification. Accepted default results } \\
\text { from Bulletin 17B }\end{array}$ & Yes. \\
\hline 176 & 05124480 & Kawishiwi River near Ely, Minn. & $04 / 30 / 1967$ & $05 / 11 / 2011$ & Accepted default results from Bulletin 17B & Yes. \\
\hline 177 & 05124500 & Isabella River near Isabella, Minn. & $06 / 03 / 1953$ & 07/04/1977 & Accepted default results from Bulletin 17B & Yes. \\
\hline 178 & 05124990 & Filson Creek in SESW sec. 24 near Winton, Minn. & $04 / 25 / 1975$ & $04 / 24 / 1985$ & $\begin{array}{l}\text { Peak flow(s) exceeded high-outlier criterion. Insufficient evidence } \\
\text { to extend historical period. Accepted default results from Bul- } \\
\text { letin 17B }\end{array}$ & Yes. \\
\hline
\end{tabular}

$17905125000 \quad$ South Kawishiwi River near Ely, Minn.

$180 \quad 05125500$

18105125550

18205126000

$183 \quad 05126210$

$184 \quad 05126500$

$185 \quad 05127000$

$186 \quad 05127205$

$\begin{array}{ll}187 & 05127207\end{array}$

$188 \quad 05127210$

$189 \quad 05127215$

$190 \quad 05127219$

$191 \quad 05127220$

$192 \quad 05127230$

$193 \quad 05127500$

$194 \quad 05128000$
Stony River near Isabella, Minn.

Stony River near Babbitt, Minn.

Dunka River near Babbitt, Minn.

South Kawishiwi River above White Iron Lake near Ely, Minn.

Bear Island River near Ely, Minn.

Kawishiwi River near Winton, Minn.

Burntside River near Ely, Minn.

Bjorkman's Creek near Ely, Minn.

Armstrong Creek near Ely, Minn.

Longstorff Creek near Ely, Minn.

Shagawa Lake tributary at Ely, Minn.

Burgo Creek near Ely, Minn.

Shagawa River at Ely, Minn.

Basswood River near Winton, Minn.

Namakan River at outlet of Lac La Croix, Ontario, Canada
04/26/1952 05/05/2011 Accepted default results from Bulletin 17B

06/03/1953 06/28/1964 Accepted default results from Bulletin 17B

04/19/1976 05/12/2011 Accepted default results from Bulletin 17B

No, out of StreamStats ${ }^{1}$ range.

Yes.

Yes.

04/22/1952 04/20/1980 Accepted default results from Bulletin 17B

Yes.

04/22/1976 05/11/2011 Low outlier(s) flagged by Bulletin 17B method. Accepted default results from EMA

06/01/1953 09/12/1977 Low outlier(s) flagged by Bulletin 17B method. Accepted default Yes. results from EMA

06/12/1906 09/17/2011 Affected by regulation, used station skew. Low outlier(s) flagged No, station skew. by Bulletin 17B method. Accepted modified results from EMA

06/14/1968 10/12/1977 Accepted default results from Bulletin 17B

Yes.

06/10/1970 05/29/1978 Insufficient peak flows

06/09/1968 05/29/1978 Accepted default results from Bulletin 17B

06/08/1968 05/29/1978 Accepted default results from Bulletin 17B

06/17/1971 05/29/1978 Insufficient peak flows

06/08/1968 05/30/1978 Peak flow(s) exceeded high-outlier criterion. Insufficient evidence Yes. to extend historical period. Accepted default results from Bulletin 17B

06/15/1968 10/14/1977 Low outlier(s) flagged by Bulletin 17B method. Accepted modi- $\quad$ Yes. fied results from EMA

07/20/1926 05/14/2011 Low outlier(s) flagged by Bulletin 17B method. Accepted default No, large drainage basin. results from EMA

05/24/1997 07/22/2010 Accepted default results from Bulletin 17B No, large drainage basin. 
Table 1-1. Streamgages in and bordering Minnesota with peak-flow data through water year 2011._Continued

[Bulletin 17B method (Interagency Advisory Committee on Water Data, 1982). EMA, expected moments algorithm (Cohn and others, 1997); **/**, month and day of occurrence unknown]

\begin{tabular}{|c|c|c|c|c|c|c|}
\hline $\begin{array}{c}\text { Map } \\
\text { number } \\
\text { (fig. 1) }\end{array}$ & $\begin{array}{l}\text { Streamgage } \\
\text { number }\end{array}$ & Streamgage name & $\begin{array}{c}\text { Start of } \\
\text { systematic } \\
\text { record }\end{array}$ & $\begin{array}{c}\text { End of } \\
\text { systematic } \\
\text { record }\end{array}$ & Peak-flow analysis comments & $\begin{array}{l}\text { Weighting of independent } \\
\text { estimates (WIE) calculated? }\end{array}$ \\
\hline 195 & 05128300 & Pike River near Gilbert, Minn. & $04 / 15 / 1966$ & $06 / 28 / 1981$ & Accepted default results from Bulletin 17B & Yes. \\
\hline 196 & 05128340 & Pike River near Biwabik, Minn. & 09/06/1977 & 07/18/1978 & Insufficient peak flows & No, insufficient peak flows. \\
\hline 197 & 05128500 & Pike River near Embarrass, Minn. & $05 / 01 / 1950$ & $04 / 23 / 1979$ & $\begin{array}{l}\text { Historical peak flow(s) detected. Extended historical period } \\
\text { back to } 1943 \text { to include } 1950 \text { peak flow based on streamgage } \\
\text { 05131000. Used a perception threshold of 2,400 from } 1943 \text { to } \\
\text { 1953, and from } 1965 \text { to } 1976 \text {. Accepted modified results from } \\
\text { EMA }\end{array}$ & Yes. \\
\hline 198 & 05128700 & Pike River tributary near Wahlsten, Minn. & $04 / 15 / 1961$ & $06 / 28 / 1981$ & $\begin{array}{l}\text { Low outlier(s) flagged by Bulletin 17B method. Accepted default } \\
\text { results from EMA }\end{array}$ & Yes. \\
\hline 199 & 05129000 & $\begin{array}{l}\text { Vermilion River below Vermilion Lake near } \\
\text { Tower, Minn. }\end{array}$ & 05/19/1912 & $05 / 02 / 1981$ & Accepted default results from Bulletin 17B & Yes. \\
\hline 200 & 05129115 & Vermilion River near Crane Lake, Minn. & 04/01/1979 & $04 / 14 / 2011$ & Accepted default results from Bulletin 17B & Yes. \\
\hline 201 & 05129290 & $\begin{array}{l}\text { Gold Portage Outlet from Kabetogama Lake near } \\
\text { Ray, Minn. }\end{array}$ & 07/09/1983 & $06 / 07 / 2011$ & $\begin{array}{l}\text { Used station skew. Low outlier(s) flagged by Bulletin 17B method. } \\
\text { Accepted default results from EMA }\end{array}$ & No, out of StreamStats ${ }^{1}$ range. \\
\hline 202 & 05129650 & Little Fork River at Cook, Minn. & $06 / 10 / 1968$ & $06 / 10 / 1984$ & $\begin{array}{l}\text { Peak flow(s) exceeded high-outlier criterion. Insufficient evidence } \\
\text { to extend historical period. Accepted default results from } \\
\text { Bulletin 17B }\end{array}$ & Yes. \\
\hline 203 & 05129710 & Johnson Creek near Britt, Minn. & $04 / 21 / 1961$ & 04/30/1975 & $\begin{array}{l}\text { Low outlier(s) flagged by Bulletin 17B method. Used a fixed low- } \\
\text { outlier threshold. Accepted modified results from EMA }\end{array}$ & Yes. \\
\hline 204 & 05130300 & Boriin Creek near Chisholm, Minn. & 09/06/1959 & $04 / 08 / 1992$ & Accepted default results from Bulletin 17B & Yes. \\
\hline 205 & 05130500 & Sturgeon River near Chisholm, Minn. & $04 / 10 / 1943$ & $04 / 17 / 2009$ & Accepted default results from Bulletin 17B & Yes. \\
\hline 206 & 05131000 & Dark River near Chisholm, Minn. & $04 / 11 / 1943$ & $04 / 20 / 1979$ & Accepted default results from Bulletin 17B & Yes. \\
\hline 207 & 05131448 & Wood Duck Creek near Nett Lake, Minn. & 05/01/1996 & $05 / 26 / 2005$ & Insufficient peak flows & No, insufficient peak flows. \\
\hline 208 & 05131455 & Nett Lake River near Nett Lake, Minn. & 05/01/1996 & $05 / 31 / 2005$ & Insufficient peak flows & No, insufficient peak flows. \\
\hline 209 & 05131500 & Little Fork River at Littlefork, Minn. & 04/19/1910 & $04 / 13 / 2011$ & Accepted default results from Bulletin 17B & Yes. \\
\hline 210 & 05131750 & Big Fork River near Bigfork, Minn. & $04 / 21 / 1973$ & $04 / 12 / 2011$ & $\begin{array}{l}\text { Peak flow(s) at or below the minimum recordable discharge. } \\
\text { Accepted default results from EMA }\end{array}$ & Yes. \\
\hline 211 & 05131878 & Bowerman Brook near Craigville, Minn. & $04 / 21 / 1979$ & $04 / 11 / 2011$ & Accepted default results from Bulletin 17B & Yes. \\
\hline 212 & 05132000 & Big Fork River at Big Falls, Minn. & 04/19/1910 & 04/13/2011 & $\begin{array}{l}\text { Low outlier(s) flagged by Bulletin 17B method. Accepted default } \\
\text { results from EMA }\end{array}$ & Yes. \\
\hline 213 & 05133500 & Rainy River at Manitou Rapids, Minn. & $10 / 23 / 1928$ & $04 / 14 / 2011$ & $\begin{array}{l}\text { Drainage area too large to use regional skew, used station skew. } \\
\text { Low outlier(s) flagged by Bulletin 17B method. Used a fixed } \\
\text { low-outlier threshold. Accepted modified results from EMA }\end{array}$ & No, large drainage basin. \\
\hline 214 & 05134100 & North Branch Rapid River near Baudette, Minn. & $03 / 31 / 1986$ & $04 / 12 / 2011$ & $\begin{array}{l}\text { Peak flow(s) exceeded high-outlier criterion. Insufficient evidence } \\
\text { to extend historical period. Low outlier(s) flagged by Bulletin } \\
\text { 17B method. Accepted default results from EMA }\end{array}$ & Yes. \\
\hline
\end{tabular}


Table 1-1. Streamgages in and bordering Minnesota with peak-flow data through water year 2011._- Continued

[Bulletin 17B method (Interagency Advisory Committee on Water Data, 1982). EMA, expected moments algorithm (Cohn and others, 1997); **/**, month and day of occurrence unknown]

\begin{tabular}{|c|c|c|c|c|c|c|}
\hline $\begin{array}{l}\text { Map } \\
\text { number } \\
\text { (fig. 1) }\end{array}$ & $\begin{array}{c}\text { Streamgage } \\
\text { number }\end{array}$ & Streamgage name & $\begin{array}{l}\text { Start of } \\
\text { systematic } \\
\text { record }\end{array}$ & $\begin{array}{l}\text { End of } \\
\text { systematic } \\
\text { record }\end{array}$ & Peak-flow analysis comments & $\begin{array}{l}\text { Weighting of independent } \\
\text { estimates (WIE) calculated? }\end{array}$ \\
\hline 215 & 05134200 & Rapid River near Baudette, Minn. & $05 / 11 / 1950$ & $04 / 11 / 2011$ & $\begin{array}{l}\text { Historical peak flow(s) detected. Extended historical period back } \\
\text { to } 1950 \text { to include } 1950 \text { peak. Low outlier(s) flagged by } \\
\text { Bulletin 17B method. Accepted modified results from EMA }\end{array}$ & Yes. \\
\hline 216 & 05137000 & Winter Road River near Baudette, Minn. & $03 / 31 / 1986$ & $04 / 12 / 2011$ & $\begin{array}{l}\text { Peak flow(s) exceeded high-outlier criterion. Insufficient evidence } \\
\text { to extend historical period. Low outlier(s) flagged by Bulletin } \\
\text { 17B method. Accepted default results from EMA }\end{array}$ & Yes. \\
\hline 217 & 05139500 & Warroad River near Warroad, Minn. & $03 / 25 / 1946$ & $04 / 07 / 1980$ & Accepted default results from Bulletin 17B & Yes. \\
\hline 218 & 05140000 & Bulldog Run near Warroad, Minn. & $03 / 22 / 1946$ & $03 / 09 / 1983$ & $\begin{array}{l}\text { Low outlier(s) flagged by Bulletin 17B method. Used a fixed low- } \\
\text { outlier threshold. Accepted modified results from EMA }\end{array}$ & Yes. \\
\hline 219 & 05140500 & East Branch Warroad River near Warroad, Minn. & $03 / 24 / 1946$ & $06 / 22 / 1983$ & Accepted default results from Bulletin 17B & Yes. \\
\hline 220 & 05200200 & Hennepin Creek near Becida, Minn. & $04 / 20 / 1979$ & $08 / 14 / 2000$ & Accepted default results from Bulletin 17B & Yes. \\
\hline 221 & 05200445 & Mississippi River at Bemidji, Minn. & $09 / 09 / 1973$ & $04 / 11 / 2011$ & Accepted default results from Bulletin 17B & Yes. \\
\hline 222 & 05200450 & Schoolcraft River near Bemidji, Minn. & $04 / 21 / 1988$ & $04 / 26 / 1989$ & Insufficient peak flows & No, insufficient peak flows. \\
\hline 223 & 05200510 & Mississippi River near Bemidji, Minn. & $04 / 18 / 1988$ & $05 / 04 / 2011$ & Accepted default results from Bulletin 17B & Yes. \\
\hline 224 & 05201500 & Mississippi River at Winnibigoshish Dam, Minn. & $06 / 22 / 1982$ & 07/06/1994 & $\begin{array}{l}\text { Affected by regulation, used station skew. Low outlier(s) flagged } \\
\text { by Bulletin 17B method. Accepted modified results from EMA }\end{array}$ & No, station skew. \\
\hline 225 & 05205200 & Boy River near Remer, Minn. & $04 / 03 / 1986$ & $06 / 28 / 2011$ & $\begin{array}{l}\text { Peak flow(s) at or below the minimum recordable discharge. } \\
\text { Accepted default results from EMA }\end{array}$ & Yes. \\
\hline 226 & 05206500 & Leech Lake River at Federal Dam, Minn. & $05 / 17 / 1982$ & $11 / 19 / 1993$ & $\begin{array}{l}\text { Affected by regulation, used station skew. Accepted modified } \\
\text { results from Bulletin 17B }\end{array}$ & No, station skew. \\
\hline 227 & 05207600 & $\begin{array}{l}\text { Mississippi River at Willow Beach at Ball Club, } \\
\text { Minn. }\end{array}$ & $06 / 16 / 2008$ & $02 / 18 / 2011$ & Insufficient peak flows & No, insufficient peak flows. \\
\hline 228 & 05210200 & Smith Creek near Hill City, Minn. & $05 / 14 / 1961$ & $06 / 11 / 2000$ & Accepted default results from Bulletin 17B & Yes. \\
\hline 229 & 05211000 & Mississippi River at Grand Rapids, Minn. & 03/20/1942 & $06 / 29 / 2011$ & $\begin{array}{l}\text { Affected by regulation, used station skew. Peak flow not used } \\
\text { because of dam break. Accepted modified results from } \\
\text { Bulletin 17B }\end{array}$ & No, station skew. \\
\hline 230 & 05212700 & Prairie River near Taconite, Minn. & $06 / 16 / 1968$ & $04 / 16 / 2011$ & Accepted default results from Bulletin 17B & Yes. \\
\hline 231 & 05216700 & O’Brien Creek near Nashwauk, Minn. & $04 / 18 / 1959$ & $04 / 18 / 1972$ & $\begin{array}{l}\text { Low outlier(s) flagged by Bulletin 17B method. Accepted default } \\
\text { results from EMA }\end{array}$ & Yes. \\
\hline 232 & 05216800 & O’Brien Creek near Pengilly, Minn. & $06 / 24 / 1964$ & $06 / 08 / 1968$ & Insufficient peak flows & No, insufficient peak flows. \\
\hline 233 & 05216820 & Initial Tailings Basin outfall near Keewatin, Minn. & $10 / 06 / 1982$ & $04 / 23 / 1985$ & Insufficient peak flows & No, insufficient peak flows. \\
\hline 234 & 05216860 & Swan River near Calumet, Minn. & 05/08/1964 & 05/01/1990 & Accepted default results from Bulletin 17B & Yes. \\
\hline 235 & 05216980 & Swan River tributary at Warba, Minn. & $04 / 21 / 1961$ & $07 / 18 / 1985$ & Accepted default results from Bulletin 17B & Yes. \\
\hline 236 & 05217000 & Swan River near Warba, Minn. & 05/01/1950 & $04 / 15 / 1969$ & $\begin{array}{l}\text { Historical peak flow(s) detected. Extended historical period back } \\
\text { to } 1950 \text { to include } 1950 \text { peak flow. Accepted modified results } \\
\text { from EMA }\end{array}$ & Yes. \\
\hline
\end{tabular}


Table 1-1. Streamgages in and bordering Minnesota with peak-flow data through water year 2011._Continued

[Bulletin 17B method (Interagency Advisory Committee on Water Data, 1982). EMA, expected moments algorithm (Cohn and others, 1997); **/**, month and day of occurrence unknown]

\begin{tabular}{|c|c|c|c|c|c|c|}
\hline $\begin{array}{l}\text { Map } \\
\text { number } \\
\text { (fig. 1) }\end{array}$ & $\begin{array}{l}\text { Streamgage } \\
\text { number }\end{array}$ & Streamgage name & $\begin{array}{l}\text { Start of } \\
\text { systematic } \\
\text { record }\end{array}$ & $\begin{array}{l}\text { End of } \\
\text { systematic } \\
\text { record }\end{array}$ & Peak-flow analysis comments & $\begin{array}{l}\text { Weighting of independent } \\
\text { estimates (WIE) calculated? }\end{array}$ \\
\hline 237 & 05217700 & Bluff Creek near Jacobson, Minn. & $05 / 14 / 1961$ & $04 / 23 / 1981$ & Accepted default results from Bulletin 17B & Yes. \\
\hline 238 & 05218000 & $\begin{array}{l}\text { Mississippi River above Sandy River near Libby, } \\
\text { Minn. }\end{array}$ & 07/18/1897 & $04 / 24 / 1927$ & $\begin{array}{l}\text { Historical peak flow in } 1927 \text { represents the largest peak flow that } \\
\text { occurred from } 1915 \text { through } 1929 \text { as noted in Water Supply } \\
\text { Paper }^{2} \text { 605. Rerun with EMA }\end{array}$ & No, station skew. \\
\hline 239 & 05219000 & Sandy River at Sandy Lake Dam, at Libby, Minn. & $04 / 27 / 1982$ & 05/07/1994 & $\begin{array}{l}\text { Affected by regulation, used station skew. Low outlier(s) flagged } \\
\text { by Bulletin 17B method. Accepted modified results from EMA }\end{array}$ & No, station skew. \\
\hline 240 & 05220500 & $\begin{array}{l}\text { Mississippi River below Sandy River near Libby, } \\
\text { Minn. }\end{array}$ & $05 / 16 / 1930$ & $06 / 12 / 1990$ & $\begin{array}{l}\text { Peak flow(s) exceeded high-outlier criterion. Insufficient evidence } \\
\text { to extend historical period. Affected by regulation, used station } \\
\text { skew. Accepted modified results from Bulletin 17B }\end{array}$ & No, station skew. \\
\hline 241 & 05220600 & Mississippi River at Palisade, Minn. & $04 / 18 / 2009$ & $03 / 18 / 2010$ & Insufficient peak flows & No, insufficient peak flows. \\
\hline 242 & 05221020 & Willow River below Palisade, Minn. & $04 / 24 / 1972$ & $04 / 10 / 2011$ & $\begin{array}{l}\text { Peak flow(s) at or below the minimum recordable discharge. } \\
\text { Accepted default results from EMA }\end{array}$ & Yes. \\
\hline 243 & 05227500 & Mississippi River at Aitkin, Minn. & $03 / 27 / 1902$ & $04 / 17 / 2011$ & $\begin{array}{l}\text { Historical peak flow(s) detected. Extended historical period back } \\
\text { to } 1888 \text { to include } 1888 \text { and } 1899 \text { peak flows. Affected by } \\
\text { regulation, used station skew. Accepted modified results from } \\
\text { Bulletin EMA. }\end{array}$ & No, station skew. \\
\hline 244 & 05229430 & Hoblin Creek near Pine River, Minn. & $07 / 08 / 2002$ & $06 / 02 / 2007$ & Insufficient peak flows & No, insufficient peak flows. \\
\hline 245 & 05229450 & Pine River near Pine River, Minn. & $03 / 28 / 1986$ & $04 / 11 / 2011$ & $\begin{array}{l}\text { Peak flow(s) at or below the minimum recordable discharge. } \\
\text { Accepted default results from EMA }\end{array}$ & Yes. \\
\hline 246 & 05231000 & $\begin{array}{l}\text { Pine River at Cross Lake Dam, at Cross Lake, } \\
\text { Minn. }\end{array}$ & $05 / 22 / 1982$ & 05/06/1994 & $\begin{array}{l}\text { Affected by regulation, used station skew. Accepted modified } \\
\text { results from Bulletin 17B }\end{array}$ & No, station skew. \\
\hline 247 & 05241500 & Rabbit River near Crosby, Minn. & $06 / 27 / 1946$ & $06 / 10 / 1963$ & $\begin{array}{l}\text { Low outlier(s) flagged by Bulletin 17B method. Accepted default } \\
\text { results from EMA }\end{array}$ & Yes. \\
\hline 248 & 05242300 & Mississippi River at Brainerd, Minn. & 04/17/1988 & 05/09/2011 & $\begin{array}{l}\text { Drainage area too large for regional skew, used station skew. } \\
\text { Accepted default results from Bulletin 17B }\end{array}$ & No, large drainage basin. \\
\hline 249 & 05242700 & Little Sand Lake outlet near Dorset, Minn. & 06/09/1932 & $06 / 11 / 1941$ & Accepted default results from Bulletin 17B & No, out of StreamStats ${ }^{1}$ range. \\
\hline 250 & 05243721 & $\begin{array}{l}\text { Straight River at County Road } 125 \text { near Osage, } \\
\text { Minn. }\end{array}$ & $05 / 22 / 1987$ & $04 / 29 / 1991$ & Insufficient peak flows & No, insufficient peak flows. \\
\hline 251 & 05243723 & $\begin{array}{l}\text { Straight River at County Road } 115 \text { near Park } \\
\text { Rapids, Minn. }\end{array}$ & $07 / 22 / 1987$ & 08/31/1989 & Insufficient peak flows & No, insufficient peak flows. \\
\hline 252 & 05243725 & Straight River near Park Rapids, Minn. & $05 / 22 / 1987$ & $04 / 13 / 2011$ & $\begin{array}{l}\text { Peak flow(s) exceeded high-outlier criterion. Insufficient evidence } \\
\text { to extend historical period. Accepted default results from } \\
\text { Bulletin 17B }\end{array}$ & Yes. \\
\hline 253 & 05244000 & Crow Wing River at Nimrod, Minn. & $05 / 29 / 1910$ & $04 / 18 / 2011$ & Accepted default results from Bulletin 17B & Yes. \\
\hline 254 & 05244100 & Kitten Creek near Sebeka, Minn. & $05 / 14 / 1961$ & $04 / 01 / 1978$ & Accepted default results from Bulletin 17B & Yes. \\
\hline 255 & 05244200 & Cat River near Nimrod, Minn. & $04 / 24 / 1961$ & $04 / 17 / 2011$ & $\begin{array}{l}\text { Peak flow(s) at or below the minimum recordable discharge. } \\
\text { Accepted default results from EMA }\end{array}$ & Yes. \\
\hline
\end{tabular}
Accepted default results from EMA 
Table 1-1. Streamgages in and bordering Minnesota with peak-flow data through water year 2011.-Continued

[Bulletin 17B method (Interagency Advisory Committee on Water Data, 1982). EMA, expected moments algorithm (Cohn and others, 1997); **/**, month and day of occurrence unknown]

\begin{tabular}{|c|c|c|c|c|c|c|}
\hline $\begin{array}{l}\text { Map } \\
\text { number } \\
\text { (fig. 1) }\end{array}$ & $\begin{array}{l}\text { Streamgage } \\
\text { number }\end{array}$ & Streamgage name & $\begin{array}{l}\text { Start of } \\
\text { systematic } \\
\text { record }\end{array}$ & $\begin{array}{l}\text { End of } \\
\text { systematic } \\
\text { record }\end{array}$ & Peak-flow analysis comments & $\begin{array}{l}\text { Weighting of independent } \\
\text { estimates (WIE) calculated? }\end{array}$ \\
\hline 256 & 05244440 & Leaf River near Aldrich, Minn. & $04 / 25 / 1972$ & $04 / 11 / 2011$ & Accepted default results from Bulletin 17B & Yes. \\
\hline 257 & 05245100 & Long Prairie River at Long Prairie, Minn. & $07 / 22 / 1972$ & $04 / 07 / 2011$ & $\begin{array}{l}\text { Low outlier(s) flagged by Bulletin 17B method. Accepted default } \\
\text { results from EMA }\end{array}$ & Yes. \\
\hline 258 & 05245190 & Eagle Creek tributary near Eagle Bend, Minn. & $06 / 24 / 2003$ & $04 / 02 / 2011$ & Insufficient peak flows & No, insufficient peak flows. \\
\hline 259 & 05245800 & Sevenmile Creek near Pillager, Minn. & 05/10/1979 & $05 / 30 / 1993$ & Accepted default results from Bulletin 17B & Yes. \\
\hline 260 & 05246000 & Crow Wing River at Pillager, Minn. & 03/19/1910 & $04 / 19 / 1950$ & Accepted default results from Bulletin 17B & No, large drainage basin. \\
\hline 261 & 05247000 & $\begin{array}{l}\text { Gull River at Gull Lake Dam near Brainerd, } \\
\text { Minn. }\end{array}$ & $04 / 21 / 1982$ & $04 / 30 / 1994$ & $\begin{array}{l}\text { Affected by regulation, used station skew. Low outlier(s) flagged } \\
\text { by Bulletin 17B method. Accepted modified results from EMA }\end{array}$ & No, station skew. \\
\hline 262 & 05247500 & Crow Wing River near Pillager, Minn. & $04 / 14 / 1965$ & $04 / 09 / 2011$ & $\begin{array}{l}\text { Affected by regulation, used station skew. Historical peak flow(s) } \\
\text { detected. Extended historical peak back to } 1924 \text { to include } 1965 \\
\text { peak flow. Accepted modified results from EMA }\end{array}$ & No, station skew. \\
\hline 263 & 05261000 & Mississippi River near Fort Ripley, Minn. & $07 / 25 / 1972$ & $05 / 05 / 2008$ & $\begin{array}{l}\text { Drainage area too large to use regional skew, used station skew. } \\
\text { Accepted default results from Bulletin 17B }\end{array}$ & No, large drainage basin. \\
\hline 264 & 05261520 & Nokasippi River near Fort Ripley, Minn. & $04 / 04 / 1986$ & $04 / 07 / 2011$ & Accepted default results from Bulletin 17B & Yes. \\
\hline 265 & 05267000 & Mississippi River near Royalton, Minn. & 08/07/1924 & $04 / 11 / 2011$ & $\begin{array}{l}\text { Drainage area too large to use regional skew, used station skew. } \\
\text { Accepted default results from Bulletin 17B }\end{array}$ & No, large drainage basin. \\
\hline 266 & 05267800 & Big Mink Creek tributary near Lastrup, Minn. & $* * / * * / 1961$ & $04 / 04 / 1980$ & $\begin{array}{l}\text { Low outlier(s) flagged by Bulletin 17B method. Accepted default } \\
\text { results from EMA }\end{array}$ & Yes. \\
\hline 267 & 05267900 & Hillman Creek near Pierz, Minn. & 05/07/1964 & $05 / 09 / 2002$ & $\begin{array}{l}\text { Peak flow(s) exceeded maximum recordable discharge. Modi- } \\
\text { fied perception thresholds on peak flow(s). Accepted modified } \\
\text { results from EMA }\end{array}$ & Yes. \\
\hline 268 & 05268000 & Platte River at Royalton, Minn. & $05 / 14 / 1930$ & $04 / 07 / 2011$ & Accepted default results from Bulletin 17B & Yes. \\
\hline 269 & 05270150 & Ashley Creek near Sauk Centre, Minn. & $09 / 25 / 1986$ & $04 / 04 / 2011$ & $\begin{array}{l}\text { Peak flow(s) at or below the minimum recordable discharge. } \\
\text { Accepted default results from EMA }\end{array}$ & Yes. \\
\hline 270 & 05270170 & Sauk River below dam at Sauk Centre, Minn. & $04 / 05 / 1989$ & $04 / 05 / 1989$ & Insufficient peak flows & No, insufficient peak flows. \\
\hline 271 & 05270300 & Sauk River tributary at Spring Hill, Minn. & $08 / 27 / 1960$ & $07 / 09 / 2000$ & $\begin{array}{l}\text { Low outlier(s) flagged by Bulletin 17B method. Accepted default } \\
\text { results from EMA }\end{array}$ & Yes. \\
\hline 272 & 05270310 & $\begin{array}{l}\text { Sauk River tributary number } 2 \text { near St. Martin, } \\
\text { Minn. }\end{array}$ & $08 / 27 / 1960$ & $04 / 23 / 1985$ & $\begin{array}{l}\text { Peak flow(s) exceeded high-outlier criterion. Historical peak } \\
\text { flow(s) detected. Extended historical period back to } 1960 \text { to } \\
\text { include } 1960 \text { peak and forward to } 2011 \text { to account for peak that } \\
\text { exceeded high-outlier criterion. Accepted modified results from } \\
\text { EMA }\end{array}$ & Yes. \\
\hline 273 & 05270500 & Sauk River near St. Cloud, Minn. & $03 / 19 / 1910$ & $07 / 20 / 2011$ & $\begin{array}{l}\text { Low outlier(s) flagged by Bulletin 17B method. Accepted default } \\
\text { results from EMA }\end{array}$ & Yes. \\
\hline 274 & 05270700 & Mississippi River at St. Cloud, Minn. & $04 / 12 / 1989$ & $04 / 13 / 2011$ & $\begin{array}{l}\text { Affected by regulation, used station skew. Accepted modified } \\
\text { results from Bulletin 17B }\end{array}$ & No, station skew. \\
\hline
\end{tabular}


Table 1-1. Streamgages in and bordering Minnesota with peak-flow data through water year 2011._Continued

[Bulletin 17B method (Interagency Advisory Committee on Water Data, 1982). EMA, expected moments algorithm (Cohn and others, 1997); **/**, month and day of occurrence unknown]

\begin{tabular}{|c|c|c|c|c|c|c|}
\hline $\begin{array}{l}\text { Map } \\
\text { number } \\
\text { (fig. 1) }\end{array}$ & $\begin{array}{l}\text { Streamgage } \\
\text { number }\end{array}$ & Streamgage name & $\begin{array}{l}\text { Start of } \\
\text { systematic } \\
\text { record }\end{array}$ & $\begin{array}{l}\text { End of } \\
\text { systematic } \\
\text { record }\end{array}$ & Peak-flow analysis comments & $\begin{array}{l}\text { Weighting of independent } \\
\text { estimates (WIE) calculated? }\end{array}$ \\
\hline 275 & 05271800 & Johnson Creek tributary at Luxemburg, Minn. & $08 / 29 / 1964$ & $03 / 29 / 1989$ & $\begin{array}{l}\text { Low outlier(s) flagged by Bulletin 17B method. Accepted default } \\
\text { results from EMA }\end{array}$ & Yes. \\
\hline 276 & 05272000 & $\begin{array}{l}\text { Johnson Creek tributary number } 2 \text { near St. Au- } \\
\text { gusta, Minn. }\end{array}$ & 05/06/1964 & $06 / 27 / 1983$ & Accepted default results from Bulletin 17B & Yes. \\
\hline 277 & 05272300 & Johnson Creek near St. Augusta, Minn. & $04 / 13 / 1964$ & $07 / 18 / 2011$ & $\begin{array}{l}\text { Peak flow(s) at or below the minimum recordable discharge. } \\
\text { Accepted default results from EMA }\end{array}$ & Yes. \\
\hline 278 & 05272950 & Clearwater River near South Haven, Minn. & $09 / 09 / 1985$ & $07 / 15 / 2011$ & $\begin{array}{l}\text { Peak flow(s) at or below the minimum recordable discharge. } \\
\text { Accepted default results from EMA }\end{array}$ & Yes. \\
\hline 279 & 05273510 & Mississippi River at Clearwater, Minn. & 07/26/1972 & $05 / 02 / 1994$ & $\begin{array}{l}\text { Drainage area too large to use regional skew, used station skew. } \\
\text { Low outlier(s) flagged by Bulletin 17B method. Accepted } \\
\text { default results from EMA }\end{array}$ & No, large drainage basin. \\
\hline 280 & 05273700 & Otsego Creek near Otsego, Minn. & $04 / 06 / 1964$ & $03 / 26 / 1989$ & Accepted default results from Bulletin 17B & Yes. \\
\hline 281 & 05274200 & Stony Brook tributary near Foley, Minn. & $06 / 28 / 1960$ & $03 / 26 / 1989$ & Accepted default results from Bulletin 17B & Yes. \\
\hline 282 & 05274700 & St. Francis River at Santiago, Minn. & $04 / 14 / 1965$ & $06 / 15 / 1981$ & Insufficient peak flows & No, insufficient peak flows. \\
\hline 283 & 05274715 & St. Francis River near Orrock, Minn. & $09 / 19 / 1980$ & $06 / 17 / 1981$ & Insufficient peak flows & No, insufficient peak flows. \\
\hline 284 & 05274750 & St. Francis River near Zimmerman, Minn. & $07 / 02 / 1983$ & $06 / 15 / 1984$ & Insufficient peak flows & No, insufficient peak flows. \\
\hline 285 & 05274900 & St. Francis River near Big Lake, Minn. & $04 / 16 / 1965$ & $04 / 28 / 1970$ & Insufficient peak flows & No, insufficient peak flows. \\
\hline 286 & 05275000 & Elk River near Big Lake, Minn. & 05/23/1911 & $04 / 10 / 2011$ & $\begin{array}{l}\text { Low outlier(s) flagged by Bulletin 17B method. Accepted default } \\
\text { results from EMA }\end{array}$ & Yes. \\
\hline 287 & 05275500 & Mississippi River at Elk River, Minn. & 04/07/1916 & $04 / 13 / 1969$ & $\begin{array}{l}\text { Historical peak flow(s) detected. Extended historical period } \\
\text { forward to } 1969 \text { to include } 1965 \text { peak flow. Size of stream is } \\
\text { too large for regional skew, used station skew. Used multiple } \\
\text { Grubbs-Beck test. Accepted modified results from EMA }\end{array}$ & No, large drainage basin. \\
\hline 288 & 05276000 & North Fork Crow River near Regal, Minn. & 04/08/1944 & $03 / 02 / 1954$ & Accepted default results from Bulletin 17B & Yes. \\
\hline 289 & 05276005 & North Fork Crow River above Paynesville, Minn. & 04/10/1996 & $06 / 22 / 1998$ & Insufficient peak flows & No, insufficient peak flows. \\
\hline 290 & 05276100 & $\begin{array}{l}\text { North Fork Crow River tributary near Paynesville, } \\
\text { Minn. }\end{array}$ & $08 / 27 / 1960$ & $03 / 15 / 1985$ & $\begin{array}{l}\text { Low outlier(s) flagged by Bulletin 17B method. Accepted default } \\
\text { results from EMA }\end{array}$ & Yes. \\
\hline 291 & 05276200 & North Fork Crow River at Paynesville, Minn. & 03/16/1973 & $07 / 13 / 2000$ & Accepted default results from Bulletin 17B & Yes. \\
\hline 292 & 05278000 & Middle Fork Crow River near Spicer, Minn. & $04 / 25 / 1949$ & $04 / 23 / 2011$ & $\begin{array}{l}\text { Low outlier(s) flagged by Bulletin 17B method. Accepted default } \\
\text { results from EMA }\end{array}$ & Yes. \\
\hline 293 & 05278120 & North Fork Crow River near Kingston, Minn. & 03/20/1985 & $07 / 18 / 2011$ & $\begin{array}{l}\text { Peak flow(s) at or below the minimum recordable discharge. } \\
\text { Accepted default results from EMA }\end{array}$ & Yes. \\
\hline 294 & 05278350 & Fountain Creek near Montrose, Minn. & $05 / 23 / 1962$ & $09 / 07 / 1991$ & $\begin{array}{l}\text { Historical peak flow(s) detected. Extended historical period } \\
\text { forward to } 1991 \text { to include } 1991 \text { peak flow. Accepted modified } \\
\text { results from EMA }\end{array}$ & Yes. \\
\hline 295 & 05278500 & South Fork Crow River at Cosmos, Minn. & 03/16/1945 & $04 / 15 / 1964$ & $\begin{array}{l}\text { Low outlier(s) flagged by Bulletin 17B method. Accepted default } \\
\text { results from EMA }\end{array}$ & Yes. \\
\hline
\end{tabular}


Table 1-1. Streamgages in and bordering Minnesota with peak-flow data through water year 2011.-Continued

[Bulletin 17B method (Interagency Advisory Committee on Water Data, 1982). EMA, expected moments algorithm (Cohn and others, 1997); **/**, month and day of occurrence unknown]

\begin{tabular}{|c|c|c|c|c|c|c|}
\hline $\begin{array}{c}\text { Map } \\
\text { number } \\
\text { (fig. 1) }\end{array}$ & $\begin{array}{c}\text { Streamgage } \\
\text { number }\end{array}$ & Streamgage name & $\begin{array}{l}\text { Start of } \\
\text { systematic } \\
\text { record }\end{array}$ & $\begin{array}{l}\text { End of } \\
\text { systematic } \\
\text { record }\end{array}$ & Peak-flow analysis comments & $\begin{array}{l}\text { Weighting of independent } \\
\text { estimates (WIE) calculated? }\end{array}$ \\
\hline 296 & 05278700 & Otter Creek near Lester Prairie, Minn. & $05 / 18 / 1961$ & $03 / 28 / 1989$ & $\begin{array}{l}\text { Low outlier(s) flagged by Bulletin 17B method. Accepted default } \\
\text { results from EMA }\end{array}$ & Yes. \\
\hline 297 & 05278750 & Otter Creek tributary near Lester Prairie, Minn. & $05 / 23 / 1962$ & 09/08/1991 & $\begin{array}{l}\text { Historical peak flow(s) detected. Extended historical period } \\
\text { forward to } 1991 \text { to include } 1991 \text { peak flow. Accepted modified } \\
\text { results from EMA }\end{array}$ & Yes. \\
\hline 298 & 05278850 & Buffalo Creek tributary near Brownton, Minn. & $* * / * * / 1961$ & 09/08/1991 & $\begin{array}{l}\text { Historical peak flow(s) detected. Extended historical period } \\
\text { forward to } 1991 \text { to include } 1991 \text { peak flow. Accepted modified } \\
\text { results from EMA }\end{array}$ & Yes. \\
\hline 299 & 05278880 & Buffalo Creek near New Auburn, Minn. & 04/03/1997 & 04/03/1997 & Insufficient peak flows & No, insufficient peak flows. \\
\hline 300 & 05278930 & Buffalo Creek near Glencoe, Minn. & 05/28/1972 & $03 / 25 / 2011$ & Accepted default results from EMA & Yes. \\
\hline 301 & 05279000 & South Fork Crow River near Mayer, Minn. & 04/07/1934 & 09/13/1991 & $\begin{array}{l}\text { Low outlier(s) flagged by Bulletin 17B method. Accepted default } \\
\text { results from EMA }\end{array}$ & Yes. \\
\hline 302 & 05279030 & $\begin{array}{l}\text { South Fork Crow River tributary near Mayer, } \\
\text { Minn. }\end{array}$ & $05 / 22 / 1962$ & $06 / 16 / 1970$ & Insufficient peak flows & No, insufficient peak flows. \\
\hline 303 & 05280000 & Crow River at Rockford, Minn. & $03 / 15 / 1910$ & $04 / 10 / 2011$ & $\begin{array}{l}\text { Low outlier(s) flagged by Bulletin 17B method. Accepted default } \\
\text { results from EMA }\end{array}$ & No, large drainage basin. \\
\hline 304 & 05280300 & $\begin{array}{l}\text { School Lake Creek tributary near St. Michael, } \\
\text { Minn. }\end{array}$ & 05/06/1964 & $06 / 16 / 1990$ & $\begin{array}{l}\text { Peak flow(s) exceeded high-outlier criterion. Insufficient evidence } \\
\text { to extend historical period. Accepted default results from EMA }\end{array}$ & Yes. \\
\hline 305 & 05283500 & Mississippi River at Anoka, Minn. & 04/05/1897 & $05 / 26 / 1913$ & River/stream similar to streamgage 05288500 . No analysis & No, large drainage basin. \\
\hline 306 & 05284100 & $\begin{array}{l}\text { Mille Lacs Lake tributary near Wealthwood, } \\
\text { Minn. }\end{array}$ & 04/18/1961 & $07 / 22 / 1972$ & $\begin{array}{l}\text { Low outlier(s) flagged by Bulletin 17B method. Accepted default } \\
\text { results from EMA }\end{array}$ & Yes. \\
\hline 307 & 05284305 & Seguchie Creek at Holt Lake outlet, near Garrison & $08 / 31 / 2004$ & $10 / 06 / 2005$ & Insufficient peak flows & No, insufficient peak flows. \\
\hline 308 & 05284310 & $\begin{array}{l}\text { Seguchie Creek above mouth near Garrison, } \\
\text { Minn. }\end{array}$ & $06 / 11 / 2005$ & $10 / 05 / 2005$ & Insufficient peak flows & No, insufficient peak flows. \\
\hline 309 & 05284600 & Robinson Brook near Onamia, Minn. & $07 / 16 / 1960$ & $04 / 23 / 1985$ & $\begin{array}{l}\text { Low outlier(s) flagged by Bulletin 17B method. Accepted default } \\
\text { results from EMA }\end{array}$ & Yes. \\
\hline 310 & 05284620 & Rum River tributary near Onamia, Minn. & $04 / 03 / 1960$ & $04 / 23 / 1985$ & Accepted default results from Bulletin 17B & Yes. \\
\hline 311 & 05284920 & Stanchfield Creek tributary near Day, Minn. & $05 / 14 / 1961$ & $04 / 23 / 1985$ & $\begin{array}{l}\text { Low outlier(s) flagged by Bulletin 17B method. Accepted default } \\
\text { results from EMA }\end{array}$ & Yes. \\
\hline 312 & 05286000 & Rum River near St. Francis, Minn. & $05 / 17 / 1930$ & $04 / 11 / 2011$ & $\begin{array}{l}\text { Low outlier(s) flagged by Bulletin 17B method. Used a fixed low- } \\
\text { outlier threshold. Accepted modified results from EMA }\end{array}$ & Yes. \\
\hline 313 & 05287890 & Elm Creek near Champlin, Minn. & 04/04/1979 & $03 / 24 / 2011$ & $\begin{array}{l}\text { Low outlier(s) flagged by Bulletin 17B method. Accepted default } \\
\text { results from EMA }\end{array}$ & Yes. \\
\hline 314 & 05288500 & Mississippi River near Anoka, Minn. & $06 / 16 / 1931$ & $04 / 11 / 2011$ & $\begin{array}{l}\text { Affected by regulation, used station skew. Low outlier(s) flagged } \\
\text { by Bulletin 17B method. Accepted modified results from EMA }\end{array}$ & No, station skew. \\
\hline 315 & 05288580 & $\begin{array}{l}\text { Rice Creek below old Highway } 8 \text { in Mounds } \\
\text { View, Minn. }\end{array}$ & $03 / 24 / 2009$ & $07 / 16 / 2011$ & Insufficient peak flows & No, insufficient peak flows. \\
\hline
\end{tabular}


Table 1-1. Streamgages in and bordering Minnesota with peak-flow data through water year 2011._Continued

[Bulletin 17B method (Interagency Advisory Committee on Water Data, 1982). EMA, expected moments algorithm (Cohn and others, 1997); **/**, month and day of occurrence unknown]

\begin{tabular}{|c|c|c|c|c|c|c|}
\hline $\begin{array}{c}\text { Map } \\
\text { number } \\
\text { (fig. 1) }\end{array}$ & $\begin{array}{c}\text { Streamgage } \\
\text { number }\end{array}$ & Streamgage name & $\begin{array}{c}\text { Start of } \\
\text { systematic } \\
\text { record }\end{array}$ & $\begin{array}{c}\text { End of } \\
\text { systematic } \\
\text { record }\end{array}$ & Peak-flow analysis comments & $\begin{array}{l}\text { Weighting of independent } \\
\text { estimates (WIE) calculated? }\end{array}$ \\
\hline 316 & 05288696 & $\begin{array}{l}\text { Shingle Creek tributary at Zane Avenue in Brook- } \\
\text { lyn Park, Minn. }\end{array}$ & $10 / 05 / 2007$ & $08 / 19 / 2009$ & Insufficient peak flows & No, insufficient peak flows. \\
\hline 317 & 05288705 & $\begin{array}{l}\text { Shingle Creek at Queen Avenue in Minneapolis, } \\
\text { Minn. }\end{array}$ & 05/19/1996 & $07 / 16 / 2011$ & $\begin{array}{l}\text { Affected by urbanization, used station skew. Accepted modified } \\
\text { results from Bulletin 17B }\end{array}$ & No, station skew. \\
\hline 318 & 05288800 & $\begin{array}{l}\text { Bassett Creek at Duluth Street in Golden Valley, } \\
\text { Minn. }\end{array}$ & 09/10/1982 & 06/03/1983 & Insufficient peak flows & No, insufficient peak flows. \\
\hline 319 & 05288810 & $\begin{array}{l}\text { North Fork Bassett Creek at 34th Avenue in } \\
\text { Crystal, Minn. }\end{array}$ & $05 / 17 / 1982$ & 03/06/1983 & Insufficient peak flows & No, insufficient peak flows. \\
\hline 320 & 05288840 & $\begin{array}{l}\text { Bassett Creek at Golden Valley Road in Golden } \\
\text { Valley, Minn. }\end{array}$ & 09/10/1982 & $03 / 06 / 1983$ & Insufficient peak flows & No, insufficient peak flows. \\
\hline 321 & 05288850 & $\begin{array}{l}\text { South Fork Bassett Creek at State Highway } 55 \text { in } \\
\text { Golden Valley, Minn. }\end{array}$ & 07/10/1982 & 07/03/1983 & Insufficient peak flows & No, insufficient peak flows. \\
\hline 322 & 05288900 & $\begin{array}{l}\text { Bassett Creek at Fruen Mill, in Minneapolis, } \\
\text { Minn. }\end{array}$ & 09/10/1982 & 03/06/1983 & Insufficient peak flows & No, insufficient peak flows. \\
\hline 323 & 05289500 & Minnehaha Creek at Minnetonka Mills, Minn. & 05/04/1954 & 06/01/1965 & Accepted default results from Bulletin 17B & Yes. \\
\hline 324 & 05289800 & $\begin{array}{l}\text { Minnehaha Creek at Hiawatha Avenue in Min- } \\
\text { neapolis, Minn. }\end{array}$ & $08 / 02 / 2006$ & $07 / 16 / 2011$ & Insufficient peak flows & No, insufficient peak flows. \\
\hline 325 & 05290000 & Little Minnesota River near Peever, S. Dak. & 03/30/1940 & $07 / 28 / 2011$ & $\begin{array}{l}\text { Low outlier(s) flagged by Bulletin 17B method. Accepted default } \\
\text { results from EMA }\end{array}$ & No, out of StreamStats ${ }^{1}$ range. \\
\hline 326 & 05291000 & Whetstone River near Big Stone City, S. Dak. & 03/08/1910 & $06 / 23 / 2011$ & $\begin{array}{l}\text { Historical peak flow(s) detected. Peak not used because of dam } \\
\text { break. Accepted default results from Bulletin 17B }\end{array}$ & No, out of StreamStats ${ }^{1}$ range. \\
\hline 327 & 05292000 & Minnesota River at Ortonville, Minn. & $03 / 31 / 1938$ & 04/09/2011 & $\begin{array}{l}\text { Affected by regulation, used station skew. Low outlier(s) flagged } \\
\text { by Bulletin 17B method. Accepted modified results from EMA }\end{array}$ & No, station skew. \\
\hline 328 & 05292500 & Minnesota River near Odessa, Minn. & $03 / 09 / 1910$ & $04 / 04 / 1963$ & $\begin{array}{l}\text { Affected by regulation, used station skew. Accepted modified } \\
\text { results from EMA }\end{array}$ & No, station skew. \\
\hline 329 & 05292704 & $\begin{array}{l}\text { North Fork Yellow Bank River near Odessa, } \\
\text { Minn. }\end{array}$ & $06 / 22 / 1991$ & $06 / 23 / 2011$ & $\begin{array}{l}\text { Low outlier(s) flagged by Bulletin 17B method. Accepted default } \\
\text { results from EMA }\end{array}$ & Yes. \\
\hline 330 & 05293000 & Yellow Bank River near Odessa, Minn. & $04 / 01 / 1940$ & $06 / 24 / 2011$ & Accepted default results from Bulletin 17B & Yes. \\
\hline 331 & 05293245 & Lake Ina tributary near Melby, Minn. & $06 / 24 / 2003$ & 05/09/2011 & Insufficient peak flows & No, insufficient peak flows. \\
\hline 332 & 05293371 & Pomme de Terre River below Elbow Lake, Minn. & 06/23/1986 & $07 / 15 / 2011$ & Accepted default results from Bulletin 17B & Yes. \\
\hline 333 & 05294000 & Pomme de Terre River at Appleton, Minn. & $03 / 26 / 1931$ & $04 / 06 / 2011$ & Accepted default results from Bulletin 17B & Yes. \\
\hline 334 & 05299100 & Lazarus Creek tributary near Canby, Minn. & $03 / 29 / 1960$ & $06 / 16 / 1990$ & Accepted default results from Bulletin 17B & Yes. \\
\hline 335 & 05299650 & Lac qui Parle River near Providence, Minn. & $04 / 14 / 2001$ & $03 / 17 / 2003$ & Insufficient peak flows & No, insufficient peak flows. \\
\hline 336 & 05299750 & Florida Creek near Burr, Minn. & $04 / 01 / 1983$ & $03 / 23 / 2011$ & Accepted default results from Bulletin 17B & Yes. \\
\hline 337 & 05299800 & $\begin{array}{l}\text { West Branch Lac qui Parle River at Dawson, } \\
\text { Minn. }\end{array}$ & $04 / 13 / 2001$ & $03 / 17 / 2003$ & Insufficient peak flows & No, insufficient peak flows. \\
\hline
\end{tabular}


Table 1-1. Streamgages in and bordering Minnesota with peak-flow data through water year 2011.-Continued

[Bulletin 17B method (Interagency Advisory Committee on Water Data, 1982). EMA, expected moments algorithm (Cohn and others, 1997); **/**, month and day of occurrence unknown]

\begin{tabular}{|c|c|c|c|c|c|c|}
\hline $\begin{array}{l}\text { Map } \\
\text { number } \\
\text { (fig. 1) }\end{array}$ & $\begin{array}{c}\text { Streamgage } \\
\text { number }\end{array}$ & Streamgage name & $\begin{array}{l}\text { Start of } \\
\text { systematic } \\
\text { record }\end{array}$ & $\begin{array}{l}\text { End of } \\
\text { systematic } \\
\text { record }\end{array}$ & Peak-flow analysis comments & $\begin{array}{l}\text { Weighting of independent } \\
\text { estimates (WIE) calculated? }\end{array}$ \\
\hline 338 & 05300000 & Lac qui Parle River near Lac qui Parle, Minn. & $03 / 12 / 1911$ & $03 / 26 / 2011$ & $\begin{array}{l}\text { Peak flow(s) of zero flow not used. Accepted default results from } \\
\text { EMA }\end{array}$ & Yes. \\
\hline 339 & 05301000 & Minnesota River near Lac qui Parle, Minn. & $04 / 04 / 1943$ & $04 / 06 / 2011$ & $\begin{array}{l}\text { Affected by regulation, used station skew. Historical peak flow(s) } \\
\text { detected. Extended historical period to encompass } 1997 \text { peak } \\
\text { flow. Peak flow(s) exceeded maximum recordable discharge. } \\
\text { Modified perception thresholds on peak flow(s). Accepted } \\
\text { modified results from EMA }\end{array}$ & No, station skew. \\
\hline 340 & 05301200 & $\begin{array}{l}\text { Minnesota River tributary near Montevideo, } \\
\text { Minn. }\end{array}$ & $03 / 29 / 1960$ & $04 / 26 / 1975$ & Accepted default results from Bulletin 17B & Yes. \\
\hline 341 & 05302500 & Little Chippewa River near Starbuck, Minn. & $04 / 16 / 1979$ & $04 / 05 / 2011$ & $\begin{array}{l}\text { Low outlier(s) flagged by Bulletin 17B method. Accepted default } \\
\text { results from EMA }\end{array}$ & Yes. \\
\hline 342 & 05302970 & Outlet Creek tributary near Starbuck, Minn. & 07/19/1962 & $10 / 18 / 1984$ & Accepted default results from Bulletin 17B & Yes. \\
\hline 343 & 05303450 & Hassel Creek near Clontarf, Minn. & $07 / 19 / 1962$ & $06 / 07 / 1980$ & $\begin{array}{l}\text { Peak flow(s) exceeded high-outlier criterion. Insufficient evidence } \\
\text { to extend historical period. Accepted default results from } \\
\text { Bulletin 17B }\end{array}$ & Yes. \\
\hline 344 & 05304000 & Shakopee Creek near Benson, Minn. & 03/30/1950 & $03 / 25 / 1954$ & Insufficient peak flows & No, insufficient peak flows. \\
\hline 345 & 05304500 & Chippewa River near Milan, Minn. & $04 / 12 / 1937$ & $04 / 05 / 2011$ & Accepted default results from Bulletin 17B & Yes. \\
\hline 346 & 05305000 & Chippewa River (TW) near Watson, Minn. & $06 / 04 / 1911$ & $04 / 05 / 2011$ & $\begin{array}{l}\text { Affected by regulation, used station skew. Low outlier(s) flagged } \\
\text { by Bulletin 17B method. Accepted modified results from EMA }\end{array}$ & No, station skew. \\
\hline 347 & 05305200 & Spring Creek near Montevideo, Minn. & $09 / 22 / 1959$ & $03 / 09 / 2000$ & $\begin{array}{l}\text { Low outlier(s) flagged by Bulletin 17B method. Peak flow(s) at } \\
\text { or below the minimum recordable discharge. Accepted default } \\
\text { results from EMA }\end{array}$ & Yes. \\
\hline 348 & 05311000 & Minnesota River at Montevideo, Minn. & $03 / 17 / 1910$ & $04 / 07 / 2011$ & $\begin{array}{l}\text { Affected by regulation, used station skew. Accepted modified } \\
\text { results from EMA }\end{array}$ & No, station skew. \\
\hline 349 & 05311200 & $\begin{array}{l}\text { North Branch Yellow Medicine River near Ivan- } \\
\text { hoe, Minn. }\end{array}$ & $04 / 13 / 1960$ & $05 / 31 / 1985$ & $\begin{array}{l}\text { Low outlier(s) flagged by Bulletin 17B method. Accepted default } \\
\text { results from EMA }\end{array}$ & Yes. \\
\hline 350 & 05311250 & $\begin{array}{l}\text { North Branch Yellow Medicine River tributary } \\
\text { near Wilno, Minn. }\end{array}$ & $04 / 13 / 1960$ & $10 / 18 / 1984$ & Accepted default results from Bulletin 17B & Yes. \\
\hline 351 & 05311300 & $\begin{array}{l}\text { North Branch Yellow Medicine tributary number } \\
2 \text { near Porter, Minn. }\end{array}$ & $04 / 13 / 1960$ & $04 / 13 / 1975$ & $\begin{array}{l}\text { Low outlier(s) flagged by Bulletin 17B method. Accepted default } \\
\text { results from EMA }\end{array}$ & Yes. \\
\hline 352 & 05311400 & $\begin{array}{l}\text { South Branch Yellow Medicine River at Min- } \\
\text { neota, Minn. }\end{array}$ & $04 / 06 / 1960$ & $03 / 22 / 2011$ & Accepted default results from Bulletin 17B & Yes. \\
\hline 353 & 05313500 & Yellow Medicine River near Granite Falls, Minn. & $06 / 14 / 1931$ & $03 / 25 / 2011$ & Accepted default results from Bulletin 17B & Yes. \\
\hline 354 & 05313780 & Hawk Creek near Maynard, Minn. & $03 / 30 / 1950$ & $06 / 05 / 2000$ & $\begin{array}{l}\text { Historical peak flow(s) detected. Insufficient evidence to extend } \\
\text { historical period. Low outlier(s) detected by Bulletin 17B } \\
\text { method. Accepted default results from EMA }\end{array}$ & Yes. \\
\hline 355 & 05313800 & $\begin{array}{l}\text { Kandiyohi County Ditch Number } 16 \text { near Blomk- } \\
\text { est, Minn. }\end{array}$ & $* * / * * / 1959$ & $05 / 27 / 1972$ & Accepted default results from Bulletin 17B & Yes. \\
\hline
\end{tabular}


Table 1-1. Streamgages in and bordering Minnesota with peak-flow data through water year 2011._Continued

[Bulletin 17B method (Interagency Advisory Committee on Water Data, 1982). EMA, expected moments algorithm (Cohn and others, 1997); **/**, month and day of occurrence unknown]

\begin{tabular}{|c|c|c|c|c|c|c|}
\hline $\begin{array}{l}\text { Map } \\
\text { number } \\
\text { (fig. 1) }\end{array}$ & $\begin{array}{c}\text { Streamgage } \\
\text { number }\end{array}$ & Streamgage name & $\begin{array}{l}\text { Start of } \\
\text { systematic } \\
\text { record }\end{array}$ & $\begin{array}{l}\text { End of } \\
\text { systematic } \\
\text { record }\end{array}$ & Peak-flow analysis comments & $\begin{array}{l}\text { Weighting of independent } \\
\text { estimates (WIE) calculated? }\end{array}$ \\
\hline 356 & 05314510 & Chetomba Creek near Renville, Minn. & $05 / 01 / 2006$ & $06 / 13 / 2008$ & Insufficient peak flows & No, insufficient peak flows. \\
\hline 357 & 05314900 & Redwood River at Ruthton, Minn. & 03/10/1959 & $07 / 21 / 1981$ & $\begin{array}{l}\text { Low outlier(s) flagged by Bulletin 17B method. Accepted default } \\
\text { results from EMA }\end{array}$ & Yes. \\
\hline 358 & 05315000 & Redwood River near Marshall, Minn. & 03/30/1940 & 03/23/2011 & $\begin{array}{l}\text { Low outlier(s) flagged by Bulletin 17B method. Accepted default } \\
\text { results from EMA }\end{array}$ & Yes. \\
\hline 359 & 05315200 & Prairie Ravine near Marshall, Minn. & $06 / 17 / 1959$ & $04 / 22 / 1985$ & $\begin{array}{l}\text { Low outlier(s) flagged by Bulletin 17B method. Accepted default } \\
\text { results from EMA }\end{array}$ & Yes. \\
\hline 360 & 05316500 & Redwood River near Redwood Falls, Minn. & $03 / 21 / 1910$ & $03 / 25 / 2011$ & Accepted default results from Bulletin 17B & Yes. \\
\hline 361 & 05316538 & Ramsey Creek near Redwood Falls, Minn. & 06/04/1991 & $03 / 24 / 2011$ & Accepted default results from Bulletin 17B & Yes. \\
\hline 362 & 05316550 & West Fork Beaver Creek near Olivia, Minn. & $06 / 26 / 1959$ & $07 / 11 / 1981$ & Accepted default results from Bulletin 17B & Yes. \\
\hline 363 & 0531656290 & $\begin{array}{l}\text { West Fork Beaver Creek at } 320 \text { Street near } \\
\text { Bechyn, Minn. }\end{array}$ & $03 / 14 / 2007$ & $03 / 14 / 2007$ & Insufficient peak flows & No, insufficient peak flows. \\
\hline 364 & 05316570 & Beaver Creek near Beaver Falls, Minn. & 05/30/1972 & $04 / 03 / 2011$ & $\begin{array}{l}\text { Peak flow(s) at or below the minimum recordable discharge. } \\
\text { Accepted default results from EMA }\end{array}$ & Yes. \\
\hline 365 & 05316580 & Minnesota River at Morton, Minn. & $04 / 15 / 2001$ & 04/04/2011 & $\begin{array}{l}\text { Drainage area too large to use regional skew, used station skew. } \\
\text { Accepted default results from Bulletin 17B }\end{array}$ & No, large drainage basin. \\
\hline 366 & 05316690 & Spring Creek tributary near Sleepy Eye, Minn. & 03/03/1966 & $07 / 26 / 1990$ & $\begin{array}{l}\text { Low outlier(s) flagged by Bulletin 17B method. Accepted default } \\
\text { results from EMA }\end{array}$ & Yes. \\
\hline 367 & 05316700 & Spring Creek near Sleepy Eye, Minn. & $05 / 31 / 1959$ & $05 / 18 / 2000$ & Accepted default results from Bulletin 17B & Yes. \\
\hline 368 & 05316770 & Minnesota River at New Ulm, Minn. & 07/28/1968 & $03 / 31 / 1976$ & Insufficient peak flows & No, insufficient peak flows. \\
\hline 369 & 05316800 & Cottonwood River tributary near Balaton, Minn. & 03/10/1959 & $03 / 11 / 1985$ & Accepted default results from Bulletin 17B & Yes. \\
\hline 370 & 05316850 & Meadow Creek tributary near Marshall, Minn. & 03/19/1961 & 03/16/1972 & Accepted default results from Bulletin 17B & Yes. \\
\hline 371 & 05316900 & Dry Creek near Jeffers, Minn. & $03 / 14 / 1961$ & $04 / 23 / 1985$ & Accepted default results from Bulletin 17B & Yes. \\
\hline 372 & 05316920 & Cottonwood River tributary near Sanborn, Minn. & $04 / 19 / 1966$ & $04 / 26 / 1994$ & Accepted default results from Bulletin 17B & Yes. \\
\hline 373 & 05316950 & Cottonwood River near Springfield, Minn. & $04 / 08 / 1969$ & $03 / 23 / 2011$ & $\begin{array}{l}\text { Peak flow(s) exceeded high-outlier criterion. Historical peak } \\
\text { flow(s) detected. Extended historical period back to } 1969 \text { to } \\
\text { include } 1969 \text { peak flow. Accepted default results from EMA }\end{array}$ & Yes. \\
\hline 374 & 05317000 & Cottonwood River near New Ulm, Minn. & $03 / 11 / 1910$ & $03 / 24 / 2011$ & $\begin{array}{l}\text { Low outlier(s) flagged by Bulletin 17B method. Accepted default } \\
\text { results from EMA }\end{array}$ & Yes. \\
\hline 375 & 05317200 & Little Cottonwood River near Courtland, Minn. & 03/03/1974 & $03 / 19 / 2010$ & Accepted default results from Bulletin 17B & Yes. \\
\hline 376 & 05317330 & $\begin{array}{l}\text { Minnesota River tributary at State Highway } 68 \\
\text { near Judson, Minn. }\end{array}$ & $04 / 11 / 2001$ & $07 / 15 / 2011$ & Insufficient peak flows & No, insufficient peak flows. \\
\hline 377 & 05317500 & Minnesota River at Judson, Minn. & 03/26/1939 & $04 / 13 / 1969$ & $\begin{array}{l}\text { Drainage area too large to use regional skew, used station skew. } \\
\text { Historical peak flow(s) detected. Extended historical period } \\
\text { back to } 1939 \text { and forward to } 1992 \text { (1993 floods) based on } \\
1965 \text { and } 1969 \text { peak flows. Accepted default results from } \\
\text { Bulletin 17B }\end{array}$ & No, large drainage basin. \\
\hline
\end{tabular}


Table 1-1. Streamgages in and bordering Minnesota with peak-flow data through water year 2011.-Continued

[Bulletin 17B method (Interagency Advisory Committee on Water Data, 1982). EMA, expected moments algorithm (Cohn and others, 1997); **/**, month and day of occurrence unknown]

\begin{tabular}{|c|c|c|c|c|c|c|}
\hline $\begin{array}{c}\text { Map } \\
\text { number } \\
\text { (fig. 1) }\end{array}$ & $\begin{array}{c}\text { Streamgage } \\
\text { number }\end{array}$ & Streamgage name & $\begin{array}{l}\text { Start of } \\
\text { systematic } \\
\text { record }\end{array}$ & $\begin{array}{l}\text { End of } \\
\text { systematic } \\
\text { record }\end{array}$ & Peak-flow analysis comments & $\begin{array}{l}\text { Weighting of independent } \\
\text { estimates (WIE) calculated? }\end{array}$ \\
\hline 378 & 05317845 & East Branch Blue Earth River near Walters, Minn. & $08 / 21 / 1979$ & 07/16/2011 & Accepted default results from Bulletin 17B & Yes. \\
\hline 379 & 05317850 & Foster Creek near Alden, Minn. & $05 / 30 / 1959$ & 07/10/1984 & Accepted default results from Bulletin 17B & Yes. \\
\hline 380 & 05318000 & $\begin{array}{l}\text { East Branch Blue Earth River near Bricelyn, } \\
\text { Minn. }\end{array}$ & 04/07/1951 & $10 / 12 / 1986$ & Accepted default results from Bulletin 17B & Yes. \\
\hline 381 & 05318100 & $\begin{array}{l}\text { East Branch Blue Earth River tributary near Blue } \\
\text { Earth, Minn. }\end{array}$ & $05 / 21 / 1960$ & $04 / 22 / 1985$ & $\begin{array}{l}\text { Low outlier(s) flagged by Bulletin 17B method. Accepted default } \\
\text { results from EMA }\end{array}$ & Yes. \\
\hline 382 & 05318195 & $\begin{array}{l}\text { Elm Creek at County Road } 103 \text { near Trimont, } \\
\text { Minn. }\end{array}$ & 06/04/1991 & 06/04/1991 & $\begin{array}{l}\text { Peak flow(s) at or below the minimum recordable discharge. } \\
\text { Accepted default results from EMA }\end{array}$ & Yes. \\
\hline 383 & 05318300 & Watonwan River near Delft, Minn. & $03 / 29 / 1960$ & $05 / 11 / 2003$ & Accepted default results from Bulletin 17B & Yes. \\
\hline 384 & 05318890 & South Fork Watonwan River near Odin, Minn. & 03/31/1979 & $03 / 27 / 1997$ & Accepted default results from Bulletin 17B & Yes. \\
\hline 385 & 05318897 & South Fork Watonwan River near Ormsby, Minn. & $03 / 31 / 1979$ & $03 / 23 / 2011$ & $\begin{array}{l}\text { Peak flow(s) at or below the minimum recordable discharge. Peak } \\
\text { flow(s) exceeded maximum recordable discharge. Modified } \\
\text { perception thresholds on peak flow(s). Accepted modified } \\
\text { results from EMA }\end{array}$ & Yes. \\
\hline 386 & 05319490 & Watonwan River above Garden City, Minn. & 06/08/1972 & 03/12/1976 & Insufficient peak flows & No, insufficient peak flows. \\
\hline 387 & 05319500 & Watonwan River near Garden City, Minn. & $04 / 03 / 1940$ & $03 / 24 / 2011$ & $\begin{array}{l}\text { Low outlier(s) flagged by Bulletin 17B method. Used a fixed low- } \\
\text { outlier threshold. Historical peak flow(s) detected. Extended } \\
\text { historical period to } 70 \text { years to encompass all three peak flows. } \\
\text { Accepted modified results from EMA }\end{array}$ & Yes. \\
\hline 388 & 05320000 & Blue Earth River near Rapidan, Minn. & $03 / 12 / 1910$ & $03 / 22 / 2011$ & $\begin{array}{l}\text { Low outlier(s) flagged by Bulletin 17B method. Used a fixed } \\
\text { low-outlier threshold. Extended historical period back to } 1910 \\
\text { to include } 1910 \text { historical peak flow. Accepted modified results } \\
\text { from EMA }\end{array}$ & No, station skew. \\
\hline 389 & 05320200 & Le Sueur River tributary near Mankato, Minn. & $05 / 30 / 1959$ & $03 / 12 / 1985$ & $\begin{array}{l}\text { Peak flow(s) exceeded high-outlier criterion. Insufficient evidence } \\
\text { to extend historical period. Low outlier(s) flagged by Bulletin } \\
\text { 17B method. Accepted default results from EMA }\end{array}$ & No, small drainage basin. \\
\hline 390 & 05320270 & Little Cobb River near Beauford, Minn. & 06/21/1996 & $03 / 20 / 2011$ & $\begin{array}{l}\text { Peak flow(s) exceeded maximum recordable discharge. Modi- } \\
\text { fied perception thresholds on peak flow(s). Accepted modified } \\
\text { results from EMA }\end{array}$ & Yes. \\
\hline 391 & 05320300 & Cobb River tributary near Mapleton, Minn. & $05 / 30 / 1959$ & $03 / 12 / 1985$ & Accepted default results from Bulletin 17B & Yes. \\
\hline 392 & 05320400 & Maple River tributary near Mapleton, Minn. & $05 / 30 / 1959$ & $03 / 13 / 1985$ & Accepted default results from Bulletin 17B & Yes. \\
\hline 393 & 05320440 & Judicial Ditch Number 49 near Amboy, Minn. & 03/30/1959 & $06 / 08 / 1972$ & Accepted default results from Bulletin 17B & Yes. \\
\hline 394 & 05320480 & Maple River near Rapidan, Minn. & 03/01/1972 & $03 / 20 / 2011$ & Accepted default results from Bulletin 17B & Yes. \\
\hline 395 & 05320500 & Le Sueur River near Rapidan, Minn. & $06 / 08 / 1940$ & $03 / 21 / 2011$ & $\begin{array}{l}\text { Low outlier(s) flagged by Bulletin 17B method. Accepted default } \\
\text { results from EMA }\end{array}$ & Yes. \\
\hline 396 & 05325000 & Minnesota River at Mankato, Minn. & $05 / 29 / 1903$ & $03 / 26 / 2011$ & $\begin{array}{l}\text { Drainage area too large to use regional skew, used station skew. } \\
\text { Low outlier(s) flagged by Bulletin 17B method. Accepted } \\
\text { default results from EMA }\end{array}$ & No, large drainage basin. \\
\hline
\end{tabular}


Table 1-1. Streamgages in and bordering Minnesota with peak-flow data through water year 2011._Continued

[Bulletin 17B method (Interagency Advisory Committee on Water Data, 1982). EMA, expected moments algorithm (Cohn and others, 1997); **/**, month and day of occurrence unknown]

\begin{tabular}{|c|c|c|c|c|c|c|}
\hline $\begin{array}{l}\text { Map } \\
\text { number } \\
\text { (fig. 1) }\end{array}$ & $\begin{array}{l}\text { Streamgage } \\
\text { number }\end{array}$ & Streamgage name & $\begin{array}{l}\text { Start of } \\
\text { systematic } \\
\text { record }\end{array}$ & $\begin{array}{l}\text { End of } \\
\text { systematic } \\
\text { record }\end{array}$ & Peak-flow analysis comments & $\begin{array}{l}\text { Weighting of independent } \\
\text { estimates (WIE) calculated? }\end{array}$ \\
\hline 397 & 05325100 & $\begin{array}{l}\text { Minnesota River tributary near North Mankato, } \\
\text { Minn. }\end{array}$ & 05/18/1961 & $04 / 30 / 1967$ & Insufficient peak flows & No, insufficient peak flows. \\
\hline 398 & 05325450 & Minnesota River tributary below St. Peter, Minn. & $02 / 25 / 2001$ & $03 / 10 / 2010$ & Insufficient peak flows & No, insufficient peak flows. \\
\hline 399 & 05326100 & Middle Branch Rush River near Gaylord, Minn. & 04/02/1979 & $06 / 01 / 2000$ & $\begin{array}{l}\text { Peak flow(s) exceeded high-outlier criterion. Insufficient evidence } \\
\text { to extend historical period. Low outlier(s) flagged by } \\
\text { Bulletin 17B method. Accepted default results from EMA }\end{array}$ & Yes. \\
\hline 400 & 05326189 & $\begin{array}{l}\text { South Branch Rush River at County Road } 63 \text { near } \\
\text { Norseland, Minn. }\end{array}$ & $04 / 03 / 2006$ & $10 / 19 / 2007$ & Insufficient peak flows & No, insufficient peak flows. \\
\hline 401 & 05327000 & High Island Creek near Henderson, Minn. & 06/06/1974 & $07 / 15 / 2011$ & $\begin{array}{l}\text { Low outlier(s) flagged by Bulletin 17B method. Accepted default } \\
\text { results from EMA }\end{array}$ & Yes. \\
\hline 402 & 05330000 & Minnesota River near Jordan, Minn. & 03/19/1935 & $03 / 28 / 2011$ & $\begin{array}{l}\text { Drainage area too large to use regional skew, used station skew. } \\
\text { Accepted default results from Bulletin 17B }\end{array}$ & No, large drainage basin. \\
\hline 403 & 05330150 & Sand Creek tributary near Montgomery, Minn. & $03 / 25 / 1961$ & $08 / 27 / 1981$ & $\begin{array}{l}\text { Low outlier(s) flagged by Bulletin 17B method. Accepted default } \\
\text { results from EMA }\end{array}$ & Yes. \\
\hline 404 & 05330200 & Rice Lake tributary near Montgomery, Minn. & $05 / 21 / 1960$ & 03/13/1985 & Accepted default results from Bulletin 17B & Yes. \\
\hline 405 & 05330300 & Sand Creek near New Prague, Minn. & $05 / 21 / 1960$ & $03 / 23 / 2011$ & Accepted default results from Bulletin 17B & Yes. \\
\hline 406 & 05330550 & $\begin{array}{l}\text { East Branch Raven Stream near New Prague, } \\
\text { Minn. }\end{array}$ & $05 / 21 / 1960$ & 03/16/1985 & $\begin{array}{l}\text { Peak flow(s) exceeded high-outlier criterion. Insufficient evidence } \\
\text { to extend historical period. Accepted default results from } \\
\text { Bulletin 17B }\end{array}$ & Yes. \\
\hline 407 & 05330600 & Sand Creek tributary number 2 near Jordan, Minn. & $05 / 21 / 1960$ & 03/13/1985 & $\begin{array}{l}\text { Peak flow(s) exceeded high-outlier criterion. Insufficient evidence } \\
\text { to extend historical period. Accepted default results from } \\
\text { Bulletin 17B }\end{array}$ & Yes. \\
\hline 408 & 05330800 & Purgatory Creek at Eden Prairie, Minn. & 03/30/1976 & 06/07/1980 & Insufficient peak flows & No, insufficient peak flows. \\
\hline 409 & 05330900 & Nine Mile Creek at Bloomington, Minn. & $07 / 16 / 1963$ & $07 / 22 / 1972$ & Accepted default results from Bulletin 17B & Yes. \\
\hline 410 & 05330920 & $\begin{array}{l}\text { Minnesota River at Fort Snelling State Park, } \\
\text { Minn. }\end{array}$ & $06 / 17 / 2004$ & $10 / 02 / 2010$ & Insufficient peak flows & No, insufficient peak flows. \\
\hline 411 & 05331000 & Mississippi River at St. Paul, Minn. & $05 / 06 / 1893$ & 03/29/2011 & $\begin{array}{l}\text { Drainage area too large to use regional skew, used station skew. } \\
\text { Accepted modified results from Bulletin 17B }\end{array}$ & No, large drainage basin. \\
\hline 412 & 05331580 & $\begin{array}{l}\text { Mississippi River below Lock and Dam Number } 2 \\
\text { at Hastings, Minn. }\end{array}$ & $04 / 13 / 1997$ & $04 / 12 / 2011$ & $\begin{array}{l}\text { Drainage area too large to use regional skew, used station skew. } \\
\text { Accepted modified results from Bulletin 17B }\end{array}$ & No, large drainage basin. \\
\hline 413 & 05333500 & St. Croix River near Danbury, Wis. & $04 / 24 / 1914$ & $08 / 03 / 2011$ & Accepted default results from Bulletin 17B & No, out of StreamStats ${ }^{1}$ range. \\
\hline 414 & 05335170 & Crooked Creek near Hinckley, Minn. & $04 / 02 / 1986$ & $08 / 03 / 2011$ & $\begin{array}{l}\text { Low outlier(s) flagged by Bulletin 17B method. Accepted default } \\
\text { results from EMA }\end{array}$ & Yes. \\
\hline 415 & 05336000 & St. Croix River near Grantsburg, Wis. & $04 / 24 / 1923$ & $03 / 16 / 2010$ & Accepted default results from Bulletin 17B & No, large drainage basin. \\
\hline 416 & 05336200 & Glaisby Brook near Kettle River, Minn. & $04 / 25 / 1960$ & $10 / 27 / 2010$ & Accepted default results from Bulletin 17B & Yes. \\
\hline 417 & 05336300 & $\begin{array}{l}\text { Moose Horn River tributary at Moose Lake, } \\
\text { Minn. }\end{array}$ & $04 / 24 / 1960$ & 07/16/1981 & Accepted default results from Bulletin 17B & Yes. \\
\hline
\end{tabular}


Table 1-1. Streamgages in and bordering Minnesota with peak-flow data through water year 2011.-Continued

[Bulletin 17B method (Interagency Advisory Committee on Water Data, 1982). EMA, expected moments algorithm (Cohn and others, 1997); **/**, month and day of occurrence unknown]

\begin{tabular}{|c|c|c|c|c|c|c|}
\hline $\begin{array}{c}\text { Map } \\
\text { number } \\
\text { (fig. 1) }\end{array}$ & $\begin{array}{l}\text { Streamgage } \\
\text { number }\end{array}$ & Streamgage name & $\begin{array}{l}\text { Start of } \\
\text { systematic } \\
\text { record }\end{array}$ & $\begin{array}{l}\text { End of } \\
\text { systematic } \\
\text { record }\end{array}$ & Peak-flow analysis comments & $\begin{array}{l}\text { Weighting of independent } \\
\text { estimates (WIE) calculated? }\end{array}$ \\
\hline 418 & 05336550 & Wolf Creek tributary near Sandstone, Minn. & $03 / 29 / 1960$ & $05 / 24 / 1989$ & $\begin{array}{l}\text { Low outlier(s) flagged by Bulletin 17B method. Accepted default } \\
\text { results from EMA }\end{array}$ & Yes. \\
\hline 419 & 05336600 & Kettle River tributary at Sandstone, Minn. & $04 / 04 / 1960$ & 06/14/1981 & Accepted default results from Bulletin 17B & Yes. \\
\hline 420 & 05336700 & Kettle River below Sandstone, Minn. & $04 / 01 / 1965$ & $10 / 28 / 2010$ & $\begin{array}{l}\text { Historical peak flow(s) detected. Extended historical period back } \\
\text { to } 1950 \text { to include } 1965 \text { peak flow. Accepted default results } \\
\text { from EMA }\end{array}$ & Yes. \\
\hline 421 & 05337400 & Knife River near Mora, Minn. & $04 / 28 / 1975$ & $04 / 23 / 2001$ & Accepted default results from Bulletin 17B & Yes. \\
\hline 422 & 05338200 & Mission Creek near Hinckley, Minn. & $03 / 29 / 1960$ & $10 / 19 / 1984$ & Accepted default results from Bulletin 17B & Yes. \\
\hline 423 & 05338500 & Snake River near Pine City, Minn. & $07 / 02 / 1914$ & $04 / 11 / 2011$ & $\begin{array}{l}\text { Historical peak flow(s) detected. Extended historical period back } \\
\text { to } 1914 \text { to include } 1950 \text { peak flow. Accepted modified results } \\
\text { from EMA }\end{array}$ & Yes. \\
\hline 424 & 05339500 & St. Croix River near Rush City, Minn. & $04 / 24 / 1923$ & 05/17/1961 & Accepted default results from Bulletin 17B & No, large drainage basin. \\
\hline 425 & 05339747 & Goose Creek at Harris, Minn. & $05 / 12 / 1986$ & $04 / 11 / 2011$ & $\begin{array}{l}\text { Low outlier(s) flagged by Bulletin 17B method. Accepted default } \\
\text { results from EMA }\end{array}$ & Yes. \\
\hline 426 & 05340000 & Sunrise River near Stacy, Minn. & $03 / 29 / 1949$ & $04 / 15 / 1965$ & Accepted default results from Bulletin 17B & Yes. \\
\hline 427 & 05340050 & Sunrise River near Lindstrom, Minn. & 03/19/1966 & 04/04/1985 & Accepted default results from Bulletin 17B & Yes. \\
\hline 428 & 05340500 & St. Croix River at St. Croix Falls, Wis. & 07/10/1902 & $10 / 30 / 2010$ & $\begin{array}{l}\text { Drainage area too large to use regional skew, used station skew. } \\
\text { Accepted modified results from Bulletin 17B }\end{array}$ & No, out of StreamStats ${ }^{1}$ range. \\
\hline 429 & 05344490 & St. Croix River at Prescott, Wis. & $04 / 29 / 2008$ & $10 / 31 / 2010$ & Insufficient peak flows & No, insufficient peak flows. \\
\hline 430 & 05344500 & Mississippi River at Prescott, Wis. & $03 / 25 / 1929$ & $04 / 12 / 2011$ & $\begin{array}{l}\text { Drainage area too large to use regional skew, used station skew. } \\
\text { Accepted default results from Bulletin 17B }\end{array}$ & No, large drainage basin. \\
\hline 431 & 05345000 & Vermillion River near Empire, Minn. & 09/18/1942 & 07/16/2011 & Accepted default results from Bulletin 17B & Yes. \\
\hline 432 & 05345900 & Vermillion River tributary near Hastings, Minn. & $12 / 28 / 1959$ & $05 / 30 / 1905$ & $\begin{array}{l}\text { Historical peak flow(s) detected. Insufficient evidence to extend } \\
\text { historical period. Accepted default results from EMA }\end{array}$ & Yes. \\
\hline 433 & 05346000 & Vermillion River at Hastings, Minn. & 03/12/1990 & 03/12/1990 & Insufficient peak flows & No, insufficient peak flows. \\
\hline 434 & 05348550 & $\begin{array}{l}\text { Cannon River below Sabre Lake near Kilkenny, } \\
\text { Minn. }\end{array}$ & $03 / 16 / 1985$ & 07/16/2011 & Accepted default results from Bulletin 17B & Yes. \\
\hline 435 & 05352700 & Turtle Creek tributary number 2 near Pratt, Minn. & $05 / 21 / 1960$ & 03/11/1985 & Accepted default results from Bulletin 17B & Yes. \\
\hline 436 & 05352800 & Turtle Creek tributary near Steele Center, Minn. & $05 / 21 / 1960$ & 03/11/1985 & $\begin{array}{l}\text { Low outlier(s) flagged by Bulletin 17B method. Accepted default } \\
\text { results from EMA }\end{array}$ & Yes. \\
\hline 437 & 05353800 & Straight River near Faribault, Minn. & 03/04/1966 & 03/23/2011 & $\begin{array}{l}\text { Low outlier(s) flagged by Bulletin 17B method. Accepted default } \\
\text { results from EMA }\end{array}$ & Yes. \\
\hline 438 & 05355024 & Cannon River at Northfield, Minn. & 03/19/1980 & 03/22/2011 & $\begin{array}{l}\text { Peak flow(s) at or below the minimum recordable discharge. Used } \\
\text { multiple Grubbs Beck test. Accepted modified results from } \\
\text { EMA }\end{array}$ & Yes. \\
\hline 439 & 05355100 & Little Cannon River tributary near Kenyon, Minn. & $06 / 23 / 1960$ & 09/21/1986 & Accepted default results from Bulletin 17B & Yes. \\
\hline
\end{tabular}


[Bulletin 17B method (Interagency Advisory Committee on Water Data, 1982). EMA, expected moments algorithm (Cohn and others, 1997); **/**, month and day of occurrence unknown]

\begin{tabular}{|c|c|c|c|c|c|c|}
\hline $\begin{array}{c}\text { Map } \\
\text { number } \\
\text { (fig. 1) }\end{array}$ & $\begin{array}{c}\text { Streamgage } \\
\text { number }\end{array}$ & Streamgage name & $\begin{array}{l}\text { Start of } \\
\text { systematic } \\
\text { record }\end{array}$ & $\begin{array}{l}\text { End of } \\
\text { systematic } \\
\text { record }\end{array}$ & Peak-flow analysis comments & $\begin{array}{l}\text { Weighting of independent } \\
\text { estimates (WIE) calculated? }\end{array}$ \\
\hline 440 & 05355150 & Pine Creek near Cannon Falls, Minn. & $03 / 27 / 1960$ & $03 / 18 / 1980$ & $\begin{array}{l}\text { Low outlier(s) flagged by Bulletin 17B method. Accepted default } \\
\text { results from EMA }\end{array}$ & Yes. \\
\hline 441 & 05355200 & Cannon River at Welch, Minn. & $06 / 04 / 1911$ & $03 / 23 / 2011$ & $\begin{array}{l}\text { Low outlier(s) flagged by Bulletin 17B method. Accepted default } \\
\text { results from EMA }\end{array}$ & Yes. \\
\hline 442 & 05355230 & Cannon River tributary near Welch, Minn. & $07 / 02 / 1960$ & $05 / 05 / 1981$ & Accepted default results from Bulletin 17B & No, small drainage basin. \\
\hline 443 & 05355250 & Mississippi River at Red Wing, Minn. & $04 / 12 / 1997$ & 04/08/1998 & Insufficient peak flows & No, insufficient peak flows. \\
\hline 444 & 05372800 & $\begin{array}{l}\text { South Fork Zumbro River on belt line at Roches- } \\
\text { ter, Minn. }\end{array}$ & $* * / * * / 1969$ & $07 / 01 / 1983$ & $\begin{array}{l}\text { Peak flow(s) exceeded high-outlier criterion. Matched period } \\
\text { of } 59 \text { years based on South Fork Zumbro River (streamgage } \\
\text { 05372995). Accepted modified results from Bulletin 17B }\end{array}$ & Yes. \\
\hline 445 & 05372930 & Bear Creek at Rochester, Minn. & $04 / 04 / 1969$ & $03 / 04 / 1983$ & $\begin{array}{l}\text { Peak flow(s) exceeded high-outlier criterion. Matched period } \\
\text { of } 59 \text { years based on South Fork Zumbro River (streamgage } \\
\text { 05372995). Accepted modified results from Bulletin 17B }\end{array}$ & Yes. \\
\hline 446 & 05372950 & $\begin{array}{l}\text { Silver Creek at Minnesota Department of Natural } \\
\text { Resources gage in Rochester, Minn. }\end{array}$ & $04 / 04 / 1969$ & 03/03/1983 & $\begin{array}{l}\text { Peak flow(s) exceeded high-outlier criterion. Matched period } \\
\text { of } 59 \text { years based on South Fork Zumbro River (streamgage } \\
\text { 05372995). Accepted modified results from Bulletin 17B }\end{array}$ & Yes. \\
\hline 447 & 05372990 & Cascade Creek at Rochester, Minn. & $04 / 04 / 1969$ & $07 / 04 / 1983$ & Accepted default results from Bulletin 17B & Yes. \\
\hline 448 & 05372995 & South Fork Zumbro River at Rochester, Minn. & $07 / 21 / 1951$ & $07 / 16 / 2011$ & Accepted default results from Bulletin 17B & Yes. \\
\hline 449 & 05373000 & South Fork Zumbro River near Rochester, Minn. & $07 / 21 / 1951$ & $07 / 12 / 1981$ & River/stream similar to streamgage 05372995 . No analysis & No analysis. \\
\hline 450 & 05373080 & Milliken Creek near Concord, Minn. & 08/29/1979 & 07/15/2011 & Accepted default results from Bulletin 17B & Yes. \\
\hline 451 & 05373350 & Zumbro River tributary near South Troy, Minn. & $06 / 17 / 1962$ & 03/11/1985 & Accepted default results from Bulletin 17B & Yes. \\
\hline 452 & 05373700 & Spring Creek near Wanamingo, Minn. & $05 / 31 / 1960$ & 09/21/1986 & Accepted default results from Bulletin 17B & Yes. \\
\hline 453 & 05373900 & Trout Brook tributary near Goodhue, Minn. & $06 / 29 / 1960$ & $09 / 21 / 1986$ & $\begin{array}{l}\text { Peak flow(s) exceeded high-outlier criterion. Insufficient evidence } \\
\text { to extend historical period. Accepted default results from } \\
\text { Bulletin 17B }\end{array}$ & Yes. \\
\hline 454 & 05374000 & Zumbro River at Zumbro Falls, Minn. & $11 / 15 / 1909$ & $03 / 24 / 2011$ & Accepted default results from Bulletin 17B & Yes. \\
\hline 455 & 05374400 & Long Creek near Potsdam, Minn. & 03/04/1966 & 07/07/1990 & Accepted default results from Bulletin 17B & Yes. \\
\hline 456 & 05374500 & Zumbro River at Theilman, Minn. & $09 / 11 / 1938$ & $04 / 02 / 1956$ & Accepted default results from Bulletin 17B & Yes. \\
\hline 457 & 05374900 & Zumbro River at Kellogg, Minn. & 03/13/1976 & 07/09/1990 & Accepted default results from Bulletin 17B & Yes. \\
\hline 458 & 05375800 & East Indian Creek tributary near Weaver, Minn. & $05 / 29 / 1962$ & $04 / 28 / 1975$ & Accepted default results from Bulletin 17B & Yes. \\
\hline 459 & 05376000 & North Fork Whitewater River near Elba, Minn. & $07 / 11 / 1940$ & $07 / 02 / 1993$ & Accepted default results from Bulletin 17B & Yes. \\
\hline 460 & 05376100 & $\begin{array}{l}\text { Middle Fork Whitewater River near St. Charles, } \\
\text { Minn. }\end{array}$ & $07 / 21 / 1991$ & 05/16/1992 & Insufficient peak flows & No, insufficient peak flows. \\
\hline 461 & 05376110 & $\begin{array}{l}\text { Middle Fork Whitewater River at State Park near } \\
\text { St. Charles, Minn. }\end{array}$ & $09 / 21 / 1986$ & $03 / 25 / 1996$ & Insufficient peak flows & No, insufficient peak flows. \\
\hline
\end{tabular}


Table 1-1. Streamgages in and bordering Minnesota with peak-flow data through water year 2011.-Continued

[Bulletin 17B method (Interagency Advisory Committee on Water Data, 1982). EMA, expected moments algorithm (Cohn and others, 1997); **/**, month and day of occurrence unknown]

\begin{tabular}{|c|c|c|c|c|c|c|}
\hline $\begin{array}{l}\text { Map } \\
\text { number } \\
\text { (fig. 1) }\end{array}$ & $\begin{array}{c}\text { Streamgage } \\
\text { number }\end{array}$ & Streamgage name & $\begin{array}{l}\text { Start of } \\
\text { systematic } \\
\text { record }\end{array}$ & $\begin{array}{c}\text { End of } \\
\text { systematic } \\
\text { record }\end{array}$ & Peak-flow analysis comments & $\begin{array}{l}\text { Weighting of independent } \\
\text { estimates (WIE) calculated? }\end{array}$ \\
\hline 463 & 05376800 & Whitewater River near Beaver, Minn. & 09/01/1938 & $08 / 23 / 1999$ & $\begin{array}{l}\text { Historical peak flow(s) detected. Extended historical period back } \\
\text { to } 1938 \text { to include } 1938 \text { peak flow. Accepted modified results } \\
\text { from EMA }\end{array}$ & Yes. \\
\hline 464 & 05377500 & Whitewater River at Beaver, Minn. & 09/01/1938 & $04 / 02 / 1956$ & $\begin{array}{l}\text { Historical peak flow(s) detected. Extended historical period back } \\
\text { to } 1938 \text { to include } 1938 \text { peak flow. Accepted modified results } \\
\text { from EMA }\end{array}$ & Yes. \\
\hline 465 & 05378220 & Garvin Brook at Stockton, Minn. & $05 / 31 / 1982$ & 07/21/1991 & Insufficient peak flows & No, insufficient peak flows. \\
\hline 466 & 05378230 & Stockton Valley Creek at Stockton, Minn. & $05 / 31 / 1982$ & $03 / 11 / 1985$ & Insufficient peak flows & No, insufficient peak flows. \\
\hline 467 & 05378235 & Garvin Brook near Minnesota City, Minn. & $05 / 31 / 1982$ & 07/21/1991 & Accepted default results from Bulletin 17B & Yes. \\
\hline 468 & 05378300 & Straight Valley Creek near Rollingstone, Minn. & $06 / 26 / 1959$ & $03 / 11 / 1985$ & Accepted default results from Bulletin 17B & Yes. \\
\hline 469 & 05378500 & Mississippi River at Winona, Minn. & 04/03/1929 & $04 / 15 / 2011$ & $\begin{array}{l}\text { Drainage area too large to use regional skew, used station skew. } \\
\text { Accepted default results from Bulletin 17B }\end{array}$ & No, large drainage basin. \\
\hline 470 & 05379000 & Gilmore Creek at Winona, Minn. & $03 / 29 / 1940$ & $07 / 21 / 1991$ & $\begin{array}{l}\text { Historical peak flow(s) detected. Extended historical period for- } \\
\text { ward to } 2011 \text { to include } 1991 \text { peak flow. Low outlier(s) flagged } \\
\text { by Bulletin 17B method. Accepted modified results from EMA }\end{array}$ & Yes. \\
\hline 471 & 05380100 & Cedar Creek near Ridgeway, Minn. & $04 / 07 / 2001$ & $03 / 23 / 2011$ & Insufficient peak flows & No, insufficient peak flows. \\
\hline 472 & 05383600 & $\begin{array}{l}\text { North Branch Root River tributary near Stewart- } \\
\text { ville, Minn. }\end{array}$ & $06 / 04 / 1958$ & $03 / 11 / 1985$ & $\begin{array}{l}\text { Low outlier(s) flagged by Bulletin 17B method. Accepted default } \\
\text { results from EMA }\end{array}$ & Yes. \\
\hline 473 & 05383700 & Mill Creek tributary near Chatfield, Minn. & $08 / 21 / 1959$ & $04 / 28 / 1975$ & Accepted default results from Bulletin 17B & Yes. \\
\hline 474 & 05383720 & Mill Creek near Chatfield, Minn. & $03 / 28 / 1962$ & $03 / 11 / 1985$ & $\begin{array}{l}\text { Low outlier(s) flagged by Bulletin 17B method. Accepted default } \\
\text { results from EMA }\end{array}$ & Yes. \\
\hline 475 & 05383850 & $\begin{array}{l}\text { South Fork Bear Creek near Grand Meadow, } \\
\text { Minn. }\end{array}$ & $03 / 28 / 1962$ & $03 / 11 / 1985$ & $\begin{array}{l}\text { Peak flow(s) exceeded high-outlier criterion. Insufficient evidence } \\
\text { to extend historical period. Accepted default results from } \\
\text { Bulletin 17B }\end{array}$ & Yes. \\
\hline 476 & 05383950 & Root River near Pilot Mound, Minn. & $05 / 12 / 2003$ & $03 / 24 / 2011$ & Insufficient peak flows & No, insufficient peak flows. \\
\hline 477 & 05384000 & Root River near Lanesboro, Minn. & 03/09/1910 & $06 / 02 / 2000$ & $\begin{array}{l}\text { Low outlier(s) flagged by Bulletin 17B method. Accepted default } \\
\text { results from EMA }\end{array}$ & Yes. \\
\hline 478 & 05384100 & Duschee Creek near Lanesboro, Minn. & $06 / 25 / 1959$ & $06 / 09 / 1984$ & Accepted default results from Bulletin 17B & Yes. \\
\hline 479 & 05384120 & South Branch Root River at Lanesboro, Minn. & $* * / * * / 1973$ & $* * / * * / 1988$ & $\begin{array}{l}\text { Peak flow(s) at or below the minimum recordable discharge. } \\
\text { Accepted default results from EMA }\end{array}$ & Yes. \\
\hline 480 & 05384150 & Root River tributary near Whalan, Minn. & $06 / 25 / 1959$ & $07 / 11 / 1981$ & Accepted default results from Bulletin 17B & No, small drainage basin. \\
\hline 481 & 05384200 & Gribben Creek near Whalen, Minn. & $06 / 25 / 1959$ & 03/11/1985 & Accepted default results from Bulletin 17B & Yes. \\
\hline 482 & 05384300 & Big Springs Creek near Arendahl, Minn. & $06 / 25 / 1959$ & $02 / 27 / 1981$ & Accepted default results from Bulletin 17B & Yes. \\
\hline 483 & 05384350 & Root River at Rushford, Minn. & $03 / 11 / 1985$ & $03 / 24 / 2011$ & $\begin{array}{l}\text { Accepted default results from Bulletin 17B. Peak flow(s) missing } \\
\text { due to insufficient rating }\end{array}$ & Yes. \\
\hline
\end{tabular}


Table 1-1. Streamgages in and bordering Minnesota with peak-flow data through water year 2011._Continued

[Bulletin 17B method (Interagency Advisory Committee on Water Data, 1982). EMA, expected moments algorithm (Cohn and others, 1997); **/**, month and day of occurrence unknown]

\begin{tabular}{|c|c|c|c|c|c|c|}
\hline $\begin{array}{c}\text { Map } \\
\text { number } \\
\text { (fig. 1) }\end{array}$ & $\begin{array}{l}\text { Streamgage } \\
\text { number }\end{array}$ & Streamgage name & $\begin{array}{c}\text { Start of } \\
\text { systematic } \\
\text { record }\end{array}$ & $\begin{array}{c}\text { End of } \\
\text { systematic } \\
\text { record }\end{array}$ & Peak-flow analysis comments & $\begin{array}{l}\text { Weighting of independent } \\
\text { estimates (WIE) calculated? }\end{array}$ \\
\hline 484 & 05384400 & Pine Creek near Arendahl, Minn. & $03 / 24 / 1959$ & $03 / 11 / 1985$ & $\begin{array}{l}\text { Low outlier(s) flagged by Bulletin 17B method. Used a fixed low- } \\
\text { outlier threshold. Accepted modified results from EMA }\end{array}$ & Yes. \\
\hline 485 & 05384500 & Rush Creek near Rushford, Minn. & $06 / 28 / 1942$ & $03 / 24 / 2011$ & $\begin{array}{l}\text { Peak flow(s) at or below the minimum recordable discharge. } \\
\text { Accepted default results from EMA }\end{array}$ & Yes. \\
\hline 486 & 05384800 & Campbell Creek near Money Creek, Minn. & $04 / 06 / 2001$ & $03 / 21 / 2011$ & Insufficient peak flows & No, insufficient peak flows. \\
\hline 487 & 05385000 & Root River near Houston, Minn. & $03 / 07 / 1910$ & $03 / 24 / 2011$ & $\begin{array}{l}\text { Low outlier(s) flagged by Bulletin 17B method. Accepted default } \\
\text { results from EMA }\end{array}$ & Yes. \\
\hline 488 & 05385500 & South Fork Root River near Houston, Minn. & $03 / 26 / 1950$ & $06 / 19 / 2011$ & $\begin{array}{l}\text { Historical peak flow(s) detected. Effect of historical peak is negli- } \\
\text { gible, no historical period modification. Accepted default results } \\
\text { from Bulletin 17B }\end{array}$ & Yes. \\
\hline 489 & 05386000 & $\begin{array}{l}\text { Root River below South Fork near Houston, } \\
\text { Minn. }\end{array}$ & 09/10/1938 & 03/27/1961 & Accepted default results from Bulletin 17B & Yes. \\
\hline 490 & 05387030 & Crooked Creek at Freeburg, Minn. & 07/04/1979 & $06 / 18 / 2011$ & $\begin{array}{l}\text { Peak flow(s) at or below the minimum recordable discharge. } \\
\text { Accepted default results from EMA }\end{array}$ & Yes. \\
\hline 491 & 05455940 & Cedar River at Lansing, Minn. & $06 / 22 / 2007$ & $03 / 23 / 2011$ & Insufficient peak flows & No, insufficient peak flows. \\
\hline 492 & 05457000 & Cedar River near Austin, Minn. & $11 / 14 / 1909$ & $03 / 23 / 2011$ & Accepted default results from Bulletin 17B & Yes. \\
\hline 493 & 05457080 & Rose Creek tributary near Dexter, Minn. & $08 / 31 / 1962$ & $03 / 11 / 1985$ & $\begin{array}{l}\text { Peak flow(s) exceeded high-outlier criterion. Insufficient evidence } \\
\text { to extend historical period. Accepted default results from Bul- } \\
\text { letin 17B }\end{array}$ & Yes. \\
\hline 494 & 05457778 & Little Cedar River near Johnsburg, Minn. & 09/09/1986 & $03 / 23 / 2011$ & $\begin{array}{l}\text { Peak flow(s) exceeded maximum recordable discharge. Modi- } \\
\text { fied perception thresholds on peak flow(s). Accepted modified } \\
\text { results from EMA }\end{array}$ & Yes. \\
\hline 495 & 05458950 & Shell Rock River tributary near Albert Lea, Minn. & $06 / 23 / 1960$ & $08 / 06 / 1968$ & Insufficient peak flows & No, insufficient peak flows. \\
\hline 496 & 05458960 & Bancroft Creek at Bancroft, Minn. & 07/06/1986 & $07 / 16 / 2011$ & Accepted default results from Bulletin 17B & Yes. \\
\hline 497 & 05474750 & $\begin{array}{l}\text { Beaver Creek tributary number } 2 \text { near Slayton, } \\
\text { Minn. }\end{array}$ & 05/17/1961 & $06 / 25 / 1985$ & $\begin{array}{l}\text { Low outlier(s) flagged by Bulletin 17B method. Accepted default } \\
\text { results from EMA }\end{array}$ & Yes. \\
\hline 498 & 05474760 & Beaver Creek tributary above Slayton, Minn. & 05/17/1961 & $10 / 15 / 1984$ & Accepted default results from Bulletin 17B & Yes. \\
\hline 499 & 05474900 & $\begin{array}{l}\text { Elk Creek on County State Aid Highway 1, near } \\
\text { Brewster, Minn. }\end{array}$ & 06/17/1996 & $07 / 12 / 2011$ & $\begin{array}{l}\text { Accepted default results from Bulletin 17B. Peak flow(s) missing } \\
\text { due to insufficient rating }\end{array}$ & Yes. \\
\hline 500 & 05475000 & $\begin{array}{l}\text { Heron Lake outlet near spillway, County Highway } \\
\text { 24, Heron Lake, Minn. }\end{array}$ & $10 / 01 / 1930$ & 06/19/1943 & $\begin{array}{l}\text { Affected by regulation, used station skew. Low outlier(s) flagged } \\
\text { by Bulletin 17B method. Accepted modified results from EMA }\end{array}$ & No, station skew. \\
\hline 501 & 05475400 & Warren Lake tributary near Windom, Minn. & 03/29/1960 & 07/07/1987 & $\begin{array}{l}\text { Peak flow(s) exceeded high-outlier criterion. Insufficient evidence } \\
\text { to extend historical period. Accepted default results from } \\
\text { Bulletin 17B }\end{array}$ & Yes. \\
\hline 502 & 05475800 & Des Moines River tributary near Jackson, Minn. & $10 / 30 / 1959$ & $04 / 23 / 1985$ & Accepted default results from Bulletin 17B & Yes. \\
\hline 503 & 05475900 & $\begin{array}{l}\text { Des Moines River tributary number } 2 \text { near Lake- } \\
\text { field, Minn. }\end{array}$ & $03 / 29 / 1960$ & $04 / 23 / 1985$ & Accepted default results from Bulletin 17B & Yes. \\
\hline
\end{tabular}


Table 1-1. Streamgages in and bordering Minnesota with peak-flow data through water year 2011.-Continued

[Bulletin 17B method (Interagency Advisory Committee on Water Data, 1982). EMA, expected moments algorithm (Cohn and others, 1997); **/**, month and day of occurrence unknown]

\begin{tabular}{|c|c|c|c|c|c|c|}
\hline $\begin{array}{c}\text { Map } \\
\text { number } \\
\text { (fig. 1) }\end{array}$ & $\begin{array}{c}\text { Streamgage } \\
\text { number }\end{array}$ & Streamgage name & $\begin{array}{l}\text { Start of } \\
\text { systematic } \\
\text { record }\end{array}$ & $\begin{array}{l}\text { End of } \\
\text { systematic } \\
\text { record }\end{array}$ & Peak-flow analysis comments & $\begin{array}{l}\text { Weighting of independent } \\
\text { estimates (WIE) calculated? }\end{array}$ \\
\hline 504 & 05476000 & Des Moines River at Jackson, Minn. & 06/30/1909 & $03 / 26 / 2011$ & $\begin{array}{l}\text { Low outlier(s) flagged by Bulletin 17B method. Accepted default } \\
\text { results from EMA }\end{array}$ & Yes. \\
\hline 505 & 05476010 & Nelson Creek at Jackson, Minn. & $05 / 30 / 1959$ & $04 / 22 / 1975$ & $\begin{array}{l}\text { Historical peak flow(s) detected. Insufficient evidence to extend } \\
\text { historical period. Accepted default results from Bulletin 17B }\end{array}$ & Yes. \\
\hline 506 & 05476100 & Story Brook near Petersburg, Minn. & $03 / 29 / 1960$ & 07/20/1972 & $\begin{array}{l}\text { Low outlier(s) flagged by Bulletin 17B method. Accepted default } \\
\text { results from EMA }\end{array}$ & Yes. \\
\hline 507 & 05476900 & Fourmile Creek near Dunnell, Minn. & $05 / 21 / 1960$ & $05 / 18 / 2000$ & Accepted default results from Bulletin 17B & Yes. \\
\hline 508 & 05476989 & East Fork Des Moines River above Ceylon, Minn. & 03/28/1986 & $07 / 14 / 2011$ & $\begin{array}{l}\text { Peak flow(s) at or below the minimum recordable discharge. } \\
\text { Accepted default results from EMA }\end{array}$ & Yes. \\
\hline 509 & 06482500 & $\begin{array}{l}\text { North Branch Pipestone Creek near Pipestone, } \\
\text { Minn. }\end{array}$ & $06 / 22 / 1991$ & $03 / 23 / 2011$ & $\begin{array}{l}\text { Accepted default results from Bulletin 17B. Peak flow(s) missing } \\
\text { due to insufficient rating }\end{array}$ & Yes. \\
\hline 510 & 06482525 & Pipestone Creek near Jasper, Minn. & $03 / 15 / 2007$ & $09 / 23 / 2010$ & Insufficient peak flows & No, insufficient peak flows. \\
\hline 511 & 06482745 & Beaver Creek at Valley Springs, S. Dak. & 09/24/1986 & $03 / 18 / 2011$ & $\begin{array}{l}\text { Accepted default results from Bulletin 17B. Peak flow(s) missing } \\
\text { due to insufficient rating }\end{array}$ & No, out of StreamStats ${ }^{1}$ range. \\
\hline 512 & 06482910 & $\begin{array}{l}\text { Rock Creek tributary at County Highway } 18 \text { near } \\
\text { Hatfield, Minn. }\end{array}$ & $06 / 26 / 2001$ & $03 / 23 / 2011$ & $\begin{array}{l}\text { Low outlier(s) flagged by Bulletin 17B method. Peak flow(s) } \\
\text { missing due to insufficient rating. Accepted default results from } \\
\text { EMA }\end{array}$ & Yes. \\
\hline 513 & 06482933 & Chanarambie Creek near Edgerton, Minn. & 04/17/1979 & $04 / 17 / 1979$ & $\begin{array}{l}\text { Peak flow(s) at or below the minimum recordable discharge. } \\
\text { Accepted default results from EMA }\end{array}$ & Yes. \\
\hline 514 & 06482950 & Mound Creek near Hardwick, Minn. & $05 / 28 / 1959$ & $05 / 16 / 1985$ & Accepted default results from Bulletin 17B & Yes. \\
\hline 515 & 06482960 & Mound Creek tributary at Hardwick, Minn. & $05 / 28 / 1959$ & $06 / 18 / 1996$ & Accepted default results from Bulletin 17B & Yes. \\
\hline 516 & 06482970 & $\begin{array}{l}\text { Champepadan Creek at County Road } 18 \text { near } \\
\text { Leota, Minn. }\end{array}$ & 06/18/1996 & $06 / 15 / 2011$ & Insufficient peak flows & No, insufficient peak flows. \\
\hline 517 & 06483000 & Rock River at Luverne, Minn. & $10 / 07 / 1911$ & $07 / 15 / 2011$ & $\begin{array}{l}\text { Historical peak flow(s) detected. Insufficient evidence to extend } \\
\text { historical period. Accepted default results from Bulletin 17B }\end{array}$ & Yes. \\
\hline 518 & 06483020 & Elk Creek near Lismore, Minn. & 06/23/1996 & $04 / 07 / 2006$ & Insufficient peak flows & No, insufficient peak flows. \\
\hline 519 & 06483050 & Rock River tributary near Luverne, Minn. & $05 / 30 / 1959$ & $03 / 11 / 1972$ & Accepted default results from Bulletin 17B & Yes. \\
\hline 520 & 06483200 & Kanaranzi Creek tributary near Lismore, Minn. & $05 / 30 / 1959$ & 07/10/1993 & $\begin{array}{l}\text { Historical peak flow(s) detected. Extended historical period for- } \\
\text { ward to } 2006 \text { based on streamgage } 06603530 \text {. Accepted default } \\
\text { results from EMA }\end{array}$ & Yes. \\
\hline 521 & 06483210 & $\begin{array}{l}\text { Kanaranzi Creek tributary number } 2 \text { near Wilm- } \\
\text { ont, Minn. }\end{array}$ & 08/14/1966 & 07/10/1993 & $\begin{array}{l}\text { Historical peak flow(s) detected. Extended historical period } \\
\text { forward to } 2006 \text { based on streamgage } 06603530 \text {. Low outlier(s) } \\
\text { flagged by Bulletin 17B method. Accepted default results from } \\
\text { EMA }\end{array}$ & Yes. \\
\hline 522 & 06483220 & $\begin{array}{l}\text { East Branch Kanaranzi Creek tributary near } \\
\text { Adrian, Minn. }\end{array}$ & $05 / 30 / 1959$ & $04 / 27 / 1964$ & Insufficient peak flows & No, insufficient peak flows. \\
\hline 523 & 06483240 & Kanaranzi Creek near Kanaranzi, Minn. & $03 / 01 / 2007$ & $11 / 20 / 2012$ & Insufficient peak flows & No, insufficient peak flows. \\
\hline
\end{tabular}


Table 1-1. Streamgages in and bordering Minnesota with peak-flow data through water year 2011._Continued

[Bulletin 17B method (Interagency Advisory Committee on Water Data, 1982). EMA, expected moments algorithm (Cohn and others, 1997); **/**, month and day of occurrence unknown]

\begin{tabular}{|c|c|c|c|c|c|c|}
\hline $\begin{array}{l}\text { Map } \\
\text { number } \\
\text { (fig. 1) }\end{array}$ & $\begin{array}{c}\text { Streamgage } \\
\text { number }\end{array}$ & Streamgage name & $\begin{array}{l}\text { Start of } \\
\text { systematic } \\
\text { record }\end{array}$ & $\begin{array}{l}\text { End of } \\
\text { systematic } \\
\text { record }\end{array}$ & Peak-flow analysis comments & $\begin{array}{l}\text { Weighting of independent } \\
\text { estimates (WIE) calculated? }\end{array}$ \\
\hline 524 & 06483350 & Little Rock River near Rushmore, Minn. & 06/06/1991 & $07 / 14 / 2011$ & $\begin{array}{l}\text { Peak flow(s) exceeded maximum recordable discharge. Modi- } \\
\text { fied perception thresholds on peak flow(s). Accepted modified } \\
\text { results from EMA }\end{array}$ & Yes. \\
\hline 525 & 06483353 & Little Rock Creek near Rushmore, Minn. & $06 / 22 / 1996$ & $07 / 14 / 2011$ & Accepted default results from Bulletin 17B & Yes. \\
\hline 526 & 06603000 & Little Sioux River near Lakefield, Minn. & 04/04/1949 & $04 / 04 / 1963$ & $\begin{array}{l}\text { Peak flow(s) exceeded high-outlier criterion. Insufficient evidence } \\
\text { to extend historical period. Accepted default results from } \\
\text { Bulletin 17B }\end{array}$ & Yes. \\
\hline 527 & 06603500 & $\begin{array}{l}\text { Jackson County Ditch Number } 11 \text { near Lakefield, } \\
\text { Minn. }\end{array}$ & $03 / 24 / 1949$ & $03 / 29 / 1960$ & Accepted default results from Bulletin 17B & Yes. \\
\hline 528 & 06603520 & $\begin{array}{l}\text { Judicial Ditch Number } 28 \text { tributary near Spafford, } \\
\text { Minn. }\end{array}$ & $05 / 30 / 1959$ & 03/11/1972 & Accepted default results from Bulletin 17B & Yes. \\
\hline 529 & 06603530 & Little Sioux River near Spafford, Minn. & $04 / 06 / 1962$ & $07 / 14 / 2011$ & Accepted default results from Bulletin 17B & Yes. \\
\hline 530 & 465839096412800 & Buffalo River East of Kragnes, Minn. & $04 / 06 / 2011$ & $04 / 06 / 2011$ & Insufficient peak flows & No, insufficient peak flows. \\
\hline
\end{tabular}

${ }^{1}$ U.S. Geological Survey (2013b).

${ }^{2}$ Grover and others (1929). 


\section{Appendix 2. Streamgage Report Files}

For each of the 409 streamgages for which peak-flow frequency analyses were completed, a streamgage report file is provided in portable document format (PDF) in the downloads directory for appendix 2 of this report. The streamgage report files present the specifications of the analyses, the results of the peak-flow analyses, and the peak-flow data. These report files summarize information from all analytical methods. The streamgage report files are described and examples provided in the "Explanation and Example of a Streamgage Report File" section of the report.

\section{Appendix 3. Streamgage Graph Files}

For each of the 409 streamgages for which peak-flow frequency analyses were completed, a streamgage graph file is provided in portable document format (PDF) in the downloads directory for appendix 3 of this report. Each streamgage graph file is composed of one page containing two graphs: one normal and one Pearson type III. Each graph has either the expected moments algorithm (EMA) or Bulletin 17B frequency estimate (with respective confidence limits) and the annual peak flows. If applicable, the graphs also indicate historical peak flows (blue dots), historical thresholds, low-outlier thresholds, and peak flows with "<" and ">" symbols (censored values). The streamgage graph files are described and examples provided in the "Explanation and Examples of Streamgage Graph Files" section of the report. 
Publishing support provided by the: Rolla Publishing Service Center

For more information concerning this publication, contact: Director, USGS Minnesota Water Science Center 2280 Woodale Drive

Mounds View, Minnesota 55112

(763) 783-3100

Or visit the Minnesota Water Science Center Web site at: http://mn.water.usgs.gov/ 



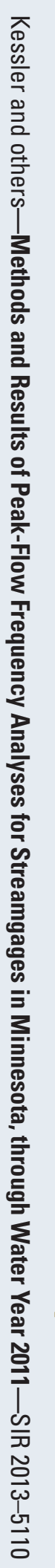

